RODRIGO VALLEJO PEREIRA

MODELAGEM MATEMÁTICA DE COPOLIMERIZAÇÃO EM EMULSÃO DE ACRILATO DE BUTILA E ESTIRENO PARA DETERMINAÇÃO DOS VALORES MÉDIOS DE PESO MOLECULAR E DISTRIBUIÇÃO DE TAMANHO DE PARTÍCULAS 
RODRIGO VALLEJO PEREIRA

\section{MODELAGEM MATEMÁTICA DE COPOLIMERIZAÇÃO EM EMULSÃO DE ACRILATO DE BUTILA E ESTIRENO PARA DETERMINAÇÃO DOS VALORES MÉDIOS DE PESO MOLECULAR E DISTRIBUIÇÃO DE TAMANHO DE PARTÍCULAS}

Tese Apresentada à Escola Politécnica da Universidade de São Paulo para obtenção do Título de Doutor em Engenharia.

Área de Concentração:

Engenharia Química

Orientador:

Prof. Titular Reinaldo Giudici. 
Este exemplar foi revisado e corrigido em relação à versão original, sob responsabilidade única do autor e com a anuência de seu orientador.

São Paulo, de de

Assinatura do autor:

Assinatura do orientador:

Pereira, Rodrigo Vallejo

Modelagem matemática de copolimerização em emulsão de acrilato de butila e estireno para determinação dos valores médios de peso molecular e distribuição de tamanho de partículas / R. V. Pereira -- versão corr. -- São Paulo, 2016.

$124 \mathrm{p}$.

Tese (Doutorado) - Escola Politécnica da Universidade de São Paulo. Departamento de Engenharia Química.

1.Modelos Matemáticos 2.Copolimerização em Emulsão 3.Distribuição de Tamanho de Partículas 4.Massa Molar Média I.Universidade de São Paulo. Escola Politécnica. Departamento de Engenharia Química II.t. 


\section{DEDICATÓRIA}

Dedico este título de doutor aos meus pais, Roberto e Vera que sempre acreditaram e me apoiaram nesta jornada chamada vida. 


\section{AGRADECIMENTOS}

Ao prof. Dr. Reinaldo Giudici pelo apoio, suporte, paciência, disponibilidade e dedicação.

Aos professores doutores Marcelo Seckler, Ardson Vianna e Galo Le Roux pelas conversas e orientações valiosas na qualificação.

Ao Prof. Dr. Efraim Cekinski e ao Prof. Dr. Marco Giulietti pelo incentivo para fazer o doutorado.

À Paula Ambrogi e Natália pela parceria e dedicação nos experimentos.

À Natura Cosméticos, em especial aos gestores Leonardo Romano e Angelica Ramos que sempre acreditaram e me incentivaram para a conclusão desta tese.

Aos amigos André e Moysés do IPT pelo sempre incentivo e valorização do trabalho.

À Carla Aparecida Santos de Morais, pela paciência e compreensão.

À Escola Politécnica da Universidade de São Paulo e todos seus colaboradores pelo apoio. 


\section{RESUMO}

Um modelo matemático da reação de copolimerização em emulsão de acrilato de butila e estireno em reator batelada e semi-batelada isotérmico foi desenvolvido e apresentou bons resultados quando comparado a experimentos disponíveis em literatura científica. O modelo contemplou a solução do balanço populacional, tanto para a distribuição de tamanho de partículas quando para a distribuição média de radicais por partícula. Contemplou-se também a solução do balanço de momentos, para obtenção da massa molar média numérica e mássica. $O$ problema descrito foi resolvido através da solução numérica de um conjunto de equações algébricodiferenciais e o balanço populacional foi resolvido pelo método dos pivots fixos. Foi possível validar com boa aderência a conversão dos monômeros, o diâmetro médio de partículas, número de partículas por litro de emulsão, número médio de radicais por partícula, a distribuição de tamanho de partículas e a massa molar média numérica e mássica ao longo do tempo para um conjunto de experimentos.

Palavras-Chave: Modelagem Matemática; Copolimerização em Emulsão; Distribuição de Tamanho de Partículas; Massa Molar Média. 


\begin{abstract}
A mathematical model of emulsion copolymerization reaction of styrene and butyl acrylate for batch and semi-batch isothermal reactor was developed and presented good results when compared to experiments available in the scientific literature. The model included the solution of the population balance for both particle size distribution and average number of radicals per particle. The balances of moments of the molecular weight distribution are solved to obtain the weight- and numberaveraged molecular weight of the polymer. The problem described was solved by numerical solution of a set of algebraic-differential equations and the population balance was solved by the method of fixed pivots. The model prediction were validated with a set of experiments with respect to the changes of monomer conversion, average particle diameter, number of particles per liter of emulsion, average number of radicals per particle, particle size distribution, number- and weight-average molecular weight during process time.
\end{abstract}

Keywords: Mathematical Modeling; Emulsion Copolymerization; Particle Size Distribution; Average Molecular Weight. 


\section{LISTA DE TABELAS}

Tabela 3.1 - Formulação típica para polimerização em emulsão. .7

Tabela 4.1 - Reações envolvidas no processo de polimerização em emulsão. 19

Tabela 4.2 - Fenômenos de transferência de radicais entre a fase aquosa e fase partícula. 20

Tabela 5.1 - Parâmetros do modelo - A: Acrilato de Butila / B: Estireno .43

Tabela 5.2 - Condições dos experimentos (ARAÚJO, 1997) .50

Tabela 5.3 - Condições dos experimentos (MARINANGELO, 2010) 52

Tabela 5.4 - Condições dos experimentos (BENYAHIA et al., 2010) 57

Tabela 6.1 - Parâmetros estimados na modelagem (ARAÚJO, 1997) …………......60

Tabela 6.2 - Parâmetros estimados na modelagem (MARINANGELO, 2010) ..........67

Tabela 6.3 - Parâmetros estimados na modelagem (BENYAHIA et al., 2010). .78

Tabela 6.4 - Parâmetros utilizados para o cálculo do efeito vítreo: (BENYAHIA et al., 2010) .78 


\section{LISTA DE FIGURAS}

Figura 3.1 - Macroprocesso de produção de materiais poliméricos - Fonte: (ASUA, 2007)

Figura 3.2 - Esquema completo dos fenômenos envolvidos polimerização em emulsão - Fonte: (THICKETT e GILBERT, 2007)

Figura 3.3 - Mecanismo: polimerização em emulsão - Fonte: (HVALA et al., 2011).12

Figura 5.1 - Fluxograma do modelo implementado

Figura 6.1 - Conversão Experimentos BSBA-1, BSBA-6, BSBA-7 e BSBA-12

Figura 6.2 - Diâmetro das Partículas para os Experimentos BSBA-1, BSBA-6, BSBA7 e BSBA-12. .62

Figura 6.3- Conversão para os Experimentos BSBA-2 e BSBA-4 .63

Figura 6.4 - Diâmetro Médio das Partículas Experimentos BSBA-2 e BSBA-4 .64

Figura 6.5 - Conversão para os Experimentos BSBA-4 e BSBA-12. .65

Figura 6.6 - Diâmetro Médio das Partículas Experimentos BSBA-4 e BSBA-12 .......66

Figura 6.7 - Conversão para os Experimentos BSBA-5 e BSBA-13. 67

Figura 6.8 - Diâmetro médio das partículas ao longo do tempo - AS23..... 68

Figura 6.9 - Número de Partículas por Litro de Emulsão - AS23. 69

Figura 6.10 - Fração de Polímero no Reator - AS23 .70

Figura 6.11 - Número de médio de radicais por partícula - AS23. .71

Figura 6.12 - Distribuição da fração volumétrica das partículas para o último instante de simulação - AS23 .72

Figura 6.13 - Distribuição da fração volumétrica das partículas para o t=180 min de simulação - AS23 72

Figura 6.14 - Distribuição da fração volumétrica das partículas para o t=270 min de simulação - AS23 .73 
Figura 6.15 - Evolução da distribuição de tamanho de partículas ao longo do tempo. .74

Figura 6.16 - Diâmetro médio das partículas ao longo do tempo - AS22 …...............75

Figura 6.17 - Número de Partículas por Litro de Emulsão - AS22 ............................76

Figura 6.18 - Fração de Polímero no reator - AS22 ……….................................76

Figura 6.19 - Número de médio de radicais por partícula - AS22 .............................77

Figura 6.20 - Conversão ao longo do Tempo para os dados de R1 a R4 …….........79

Figura 6.21 - Diâmetro Médio das Partículas para os experimentos de R1 a R4 ......80

Figura 6.22 - Massa molar média numérica dos experimentos de R1 a R4 ..............81

Figura 6.23 - Massa molar média mássica dos experimentos de R1 a R4 ..............82 


\section{LISTA DE ABREVIAÇÕES E NOTAÇÕES}

[A] Concentração de Agente Transferência de Cadeia $\left(\mathrm{kmol} \mathrm{m}^{-3}\right)$

$[A]_{p}^{e}$

Concentração de Agente Transferência de Cadeia nas partículas em equilíbrio termodinâmico $\left(\mathrm{kmol} \mathrm{m}^{-3}\right)$

$A_{p T} \quad$ Área total das partículas $\left(\mathrm{m}^{2}\right)$

$A_{d} \quad$ Área da gota de monômero $\left(\mathrm{m}^{2}\right)$

$a_{1} \quad$ Constante a1 para cálculo do efeito gel

$a_{2} \quad$ Constante a2 para cálculo do efeito gel

$a_{s} \quad$ Área coberta por um $\mathrm{kmol}$ de emulsificante $\left(\mathrm{m}^{2} \mathrm{kmol}^{-1}\right)$

$c \quad$ Constante para ajustes para calculo de $\tilde{n}(r, t)$

CMC Concentração Micelar Crítica $\left(\mathrm{kmol} \mathrm{m}^{-3}\right)$

$D_{i} \quad$ Difusividade do monômero $i\left(\mathrm{~m}^{2} \mathrm{~s}^{-1}\right)$

$f \quad$ Eficiência de decomposição do iniciador

$f_{b} \quad$ Fração molar total do monômero de estireno.

$f_{\text {kemic }} \quad$ Fator de eficiência de captura dos radicais pelas micelas

$f_{k e} \quad$ Fator de eficiência de captura de radicais pelas partículas

$F(r, t) \quad$ Função de densidade populacional para as partículas

$g \quad$ Fator do efeito gel

$K_{d}^{i} \quad$ Coeficiente de partição entre monômero $i$ e as gotas

$K_{p}^{i} \quad$ Coeficiente de partição entre monômero $i$ e as partículas 


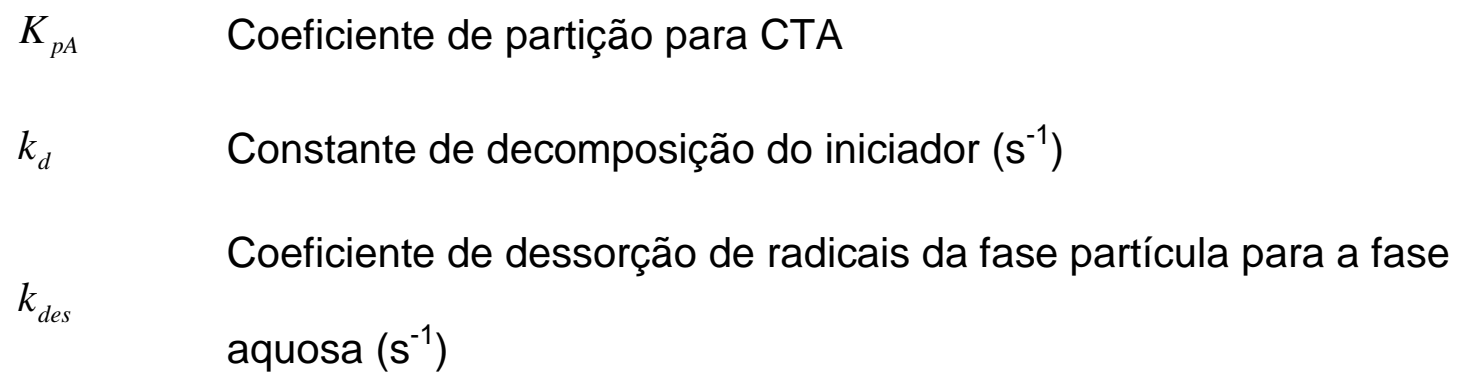

Constante média da taxa de transferência para monômero do radical tipo $i\left(\mathrm{~m}^{3} \mathrm{kmol}^{-1} \mathrm{~s}^{-1}\right)$

$k_{f p} \quad$ Constante média da taxa de transferência para polímero $\left(\mathrm{m}^{3} \mathrm{kmol}^{-1} \mathrm{~s}^{-1}\right)$

$k_{d M i}(r) \quad$ Constante da taxa de saída de radicais para partícula de raio $r\left(\mathrm{~s}^{-1}\right)$ Constante da taxa de captura de radicais $i$ pelas partículas $\left(\mathrm{m}^{3} \mathrm{kmol}^{-1} \mathrm{~s}^{-}\right.$ $k_{e, i}$ 1)

$k_{\text {emic, },} \quad$ Constante da taxa de captura de radicais $i$ pelas micelas $\left(\mathrm{m}^{3} \mathrm{kmol}^{-1} \mathrm{~s}^{-1}\right)$ Constante da taxa de transferência para monômero entre o radical tipo $i$ $k_{f m i j}$ com o monômero $j\left(\mathrm{~m}^{3} \mathrm{kmol}^{-1} \mathrm{~s}^{-1}\right)$ Constante média da taxa de transferência entre CTA e monômero $\left(\mathrm{m}^{3}\right.$ $k_{f A}$ $\left.\mathrm{kmol}^{-1} \mathrm{~s}^{-1}\right)$

Coeficiente de transferência de massa do CTA entre as gotas e a fase $k_{f A, a q}$ aquosa $\left(\mathrm{m} \mathrm{s}^{-1}\right)$

Constante taxa de transferência entre CTA e monômero do radical tipo $i$ $k_{f A i}$ $\left(\mathrm{m}^{3} \mathrm{kmol}^{-1} \mathrm{~s}^{-1}\right)$

$k_{o i} \quad$ Coeficiente da taxa de dessorção de radicais monoméricos do tipo $i\left(\mathrm{~s}^{-1}\right)$

$k_{p i} \quad$ Constante da taxa de propagação do monômero $i\left(\mathrm{~m}^{3} \mathrm{~mol}^{-1} \mathrm{~s}^{-1}\right)$ Constante da taxa de propagação entre o radical $i$ com o monômero $j$ $k_{p i j}$ $\left(\mathrm{m}^{3} \mathrm{kmol}^{-1} \mathrm{~s}^{-1}\right)$ 
Constante da taxa média de propagação para o monômero $i\left(\mathrm{~m}^{3} \mathrm{kmol}^{-1}\right.$

$\left.s^{-1}\right)$

$k_{t} \quad$ Constante média da taxa de terminação $\left(\mathrm{m}^{3} \mathrm{kmol}^{-1} \mathrm{~s}^{-1}\right)$

$k_{t c} \quad$ Constante taxa de terminação por combinação $\left(\mathrm{m}^{3} \mathrm{kmol}^{-1} \mathrm{~s}^{-1}\right)$

$k_{t d} \quad$ Constante taxa de terminação por desproporcionamento $\left(\mathrm{m}^{3} \mathrm{kmol}^{-1} \mathrm{~s}^{-1}\right)$

H Função de Heaviside

[I] Concentração de iniciador no reator $\left(\mathrm{mol} \mathrm{m}^{-3}\right)$

$j_{\text {crit }} \quad$ Tamanho crítico dos oligoradicais

$M_{n} \quad$ Massa molar média numérica $\left(\mathrm{kg} \mathrm{kmol}^{-1}\right)$

$M_{w} \quad$ Massa molar média mássica $\left(\mathrm{kg} \mathrm{kmol}^{-1}\right)$

$\left[M_{i}\right] \quad$ Concentração de monômero $i$ no reator $\left(\mathrm{kmol} \mathrm{m}^{-3}\right)$

$\left[M_{i o}\right] \quad$ Concentração total de monômero $i$ no reator $\left(\mathrm{kmol} \mathrm{m}^{-3}\right)$

$M M_{i} \quad$ Massa molar do monômero $i\left(\mathrm{~kg} \mathrm{kmol}^{-1}\right)$

$\widetilde{n}(r, t) \quad$ Número médio de radicais por partícula em função do raio e do tempo

$N_{A} \quad$ Número de Avogadro $\left(\mathrm{kmol}^{-1}\right)$

$N_{\text {mic }} \quad$ Número de micelas

$N_{p} \quad$ Número de partículas no reator

$p_{i} \quad$ Probabilidade do radical tipo $i$ dentro das partículas

$Q_{f} \quad$ Vazão volumétrica de alimentação do reator $\left(\mathrm{m}^{3} \mathrm{~s}^{-1}\right)$

$r \quad$ Raio da partícula $(\mathrm{m})$

$r_{\text {nuc }} \quad$ Raio de nucleação da partícula. (m) 


$$
\begin{aligned}
& r_{\text {mic }} \quad \text { Raio da micela }(\mathrm{m}) \\
& r_{\max } \quad \text { Raio máximo que uma partícula pode atingir }(\mathrm{m}) \\
& r_{s} \quad \text { Raio da partícula inchada }(\mathrm{m}) \\
& r_{\text {Superior }} \quad \text { Raio máximo formado entre partículas menores que podem coagular } \\
& r_{\text {corte }} \quad \text { Raio mínimo das partículas que podem sofrem coagulação } \\
& r_{i j} \quad \text { Razão de reatividade } \\
& {\left[\dot{R}_{i}\right] \quad \text { Concentração de radicais de tamanho } i\left(\mathrm{kmol} \mathrm{m}^{-3}\right)} \\
& {\left[\dot{R}_{T}\right] \quad \text { Concentração total de radicais }\left(\mathrm{kmol} \mathrm{m}^{-3}\right)} \\
& \Re_{\text {Hom }} \quad \text { Taxa de nucleação homogênea }\left(\mathrm{s}^{-1}\right) \\
& \mathfrak{R}_{\text {Coag }} \quad \text { Taxa de coagulação de partículas }\left(\mathrm{s}^{-1}\right) \\
& \Re_{p i} \quad \text { Taxa de polimerização de monômeros do tipo } i\left(\mathrm{kmol} \mathrm{s}^{-1}\right) \\
& \Re_{f i} \quad \text { Taxa de transferência de cadeia para monômeros do tipo } i\left(\mathrm{kmol} \mathrm{s}^{-1}\right) \\
& \Re_{\text {Nuc }} \quad \text { Taxa de nucleação de partículas }\left(\mathrm{s}^{-1}\right) \\
& \Re_{\text {Mic }} \quad \text { Taxa de nucleação micelar }\left(\mathrm{s}^{-1}\right) \\
& \Re_{\text {Esgotamento }} \\
& \text { Taxa de esgotamento de partículas de tamanho } r \text { devido à coagulação } \\
& \left(\mathrm{s}^{-1}\right) \\
& \Re_{\text {Formasăo }} \text { Taxa de formação de partículas de raio } r \text { devido à coagulação }\left(\mathrm{s}^{-1}\right) \\
& \text { [S] Concentração de emulsificante no reator }\left(\mathrm{mol} \mathrm{m}^{-3}\right) \\
& v_{p}(r) \quad \text { Volume de uma partícula de tamanho } r\left(\mathrm{~m}^{3}\right) \\
& V_{a q} \quad \text { Volume da fase aquosa }\left(\mathrm{m}^{3}\right) \\
& V_{a q}^{i} \quad \text { Volume de monômero } i \text { na fase aquosa }\left(\mathrm{m}^{3}\right)
\end{aligned}
$$




$\begin{array}{ll}V_{d} & \text { Volume das gotas }\left(\mathrm{m}^{3}\right) \\ V_{d}^{i} & \text { Volume do monômero } i \text { nas gotas }\left(\mathrm{m}^{3}\right) \\ V_{p} & \text { Volume das partículas }\left(\mathrm{m}^{3}\right) \\ V_{p o l} & \text { Volume de polímero no reator }\left(\mathrm{m}^{3}\right) \\ V_{p}^{i} & \text { Volume de monômero } i \text { nas partículas }\left(\mathrm{m}^{3}\right) \\ V_{R} & \text { Volume do reator }\left(\mathrm{m}^{3}\right) \\ V_{H_{2} O} & \text { Volume de água no reator }\left(\mathrm{m}^{3}\right) \\ t & \text { Tempo (s) } \\ y_{i} & \text { Composição de polímero relacionada ao monômero tipo } i \\ Y_{B A} & \text { Composição de acrilato de butila em relação ao polímero formado } \\ z & \text { Tamanho do oligoradical que permite que o mesmo entre nas partículas } \\ & \text { antes de precipitar }\end{array}$

Símbolos Gregos:
$\beta_{i}$
Probabilidade de um radical do tipo $i$ reagir na fase aquosa por propagação ou terminação.
$\beta^{\prime} \quad$ Taxa de coagulação intrínseca dependente entre partículas de raio $r^{\prime}$ e $r^{\prime \prime}$
$\varphi \quad$ Fração de água no reator
$\sigma \quad$ Taxa de absorção de radicais nas Partículas $\left(\mathrm{s}^{-1}\right)$
$\theta \quad$ Coeficiente relacionado ao grau de saturação das partículas
$\rho_{i} \quad$ Densidade do monômero $i\left(\mathrm{~kg} \mathrm{~m}^{-3}\right)$ 
$\rho_{p o l} \quad$ Densidade do polímero $\left(\mathrm{kg} \mathrm{m}^{-3}\right)$

$\Phi_{p}^{p} \quad$ Fração de polímero nas partículas

$\gamma \quad$ Número de moléculas para recobrir uma micela

$\lambda_{k} \quad$ Momento dos radicais de ordem $\mathrm{k}$

$\tau$ Razão entre as constantes de combinação e desproporcionamento

$\mu_{k} \quad$ Momento dos polímeros mortos de ordem $\mathrm{k}$

Subscritos:

$a q \quad$ Fase aquosa

f $\quad$ Corrente de alimentação

$p \quad$ Fase polímero (partículas)

KPS Persulfato de potássio

NPS Persulfato de amônio 


\section{SUMÁRIO}

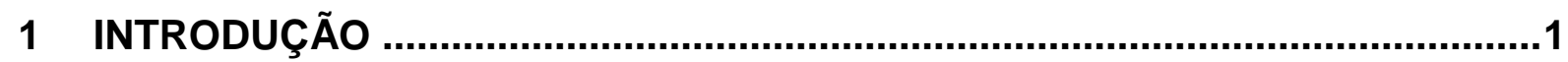

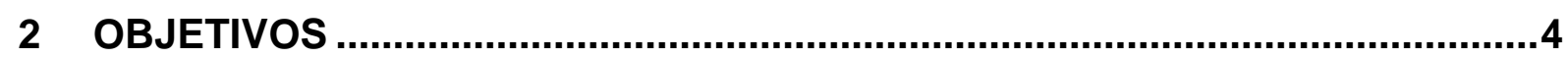

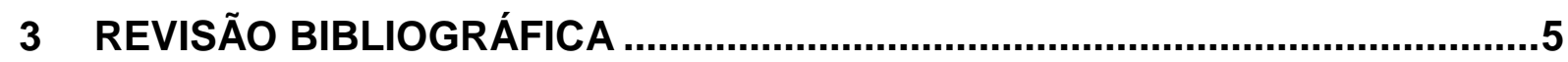

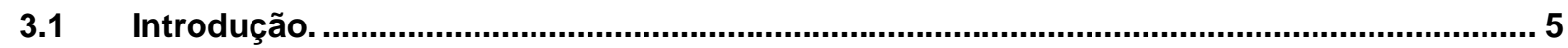

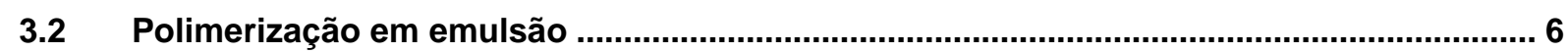

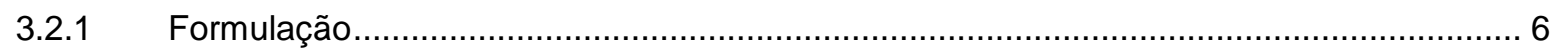

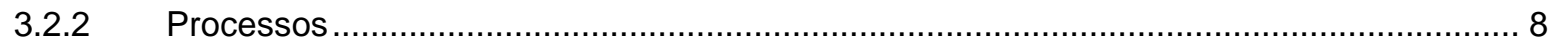

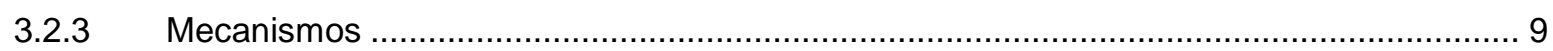

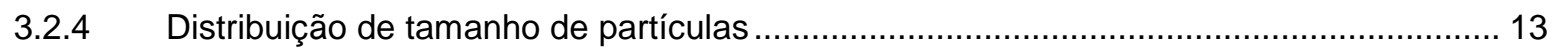

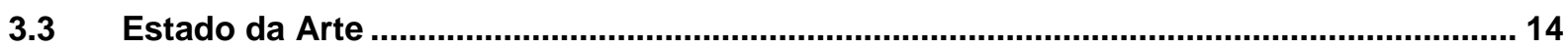

4 MODELAGEM MATEMÁTICA ...................................................................16

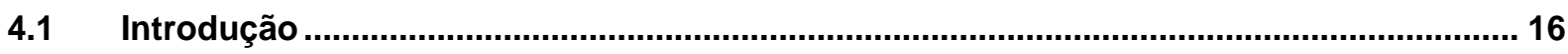

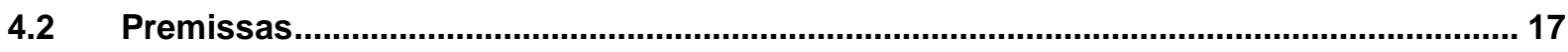

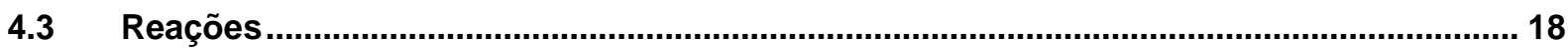

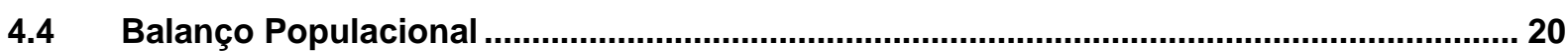

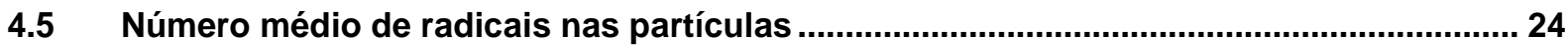

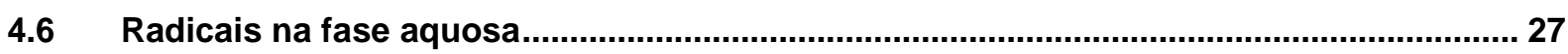

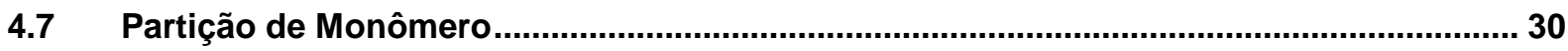

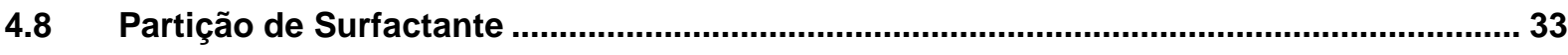

4.9 Partição de Agente de Transferência de Cadeia.............................................................. 34

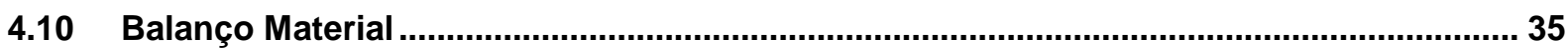

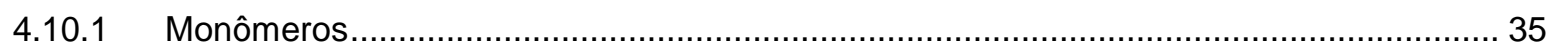

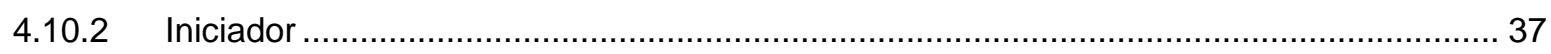

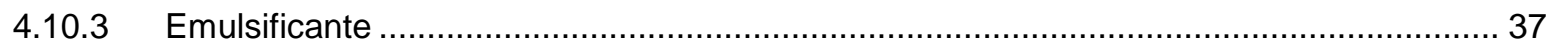

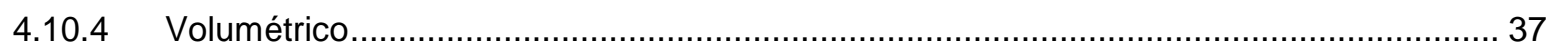

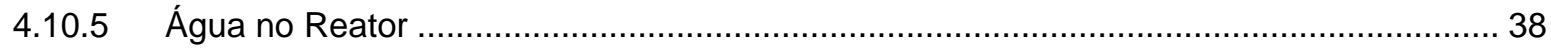

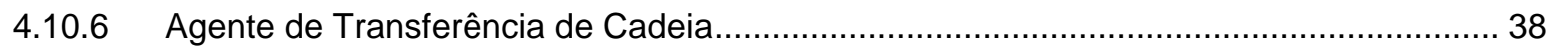

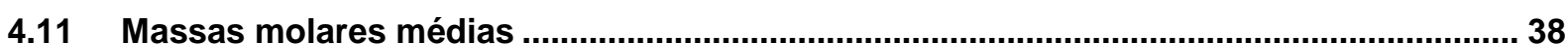

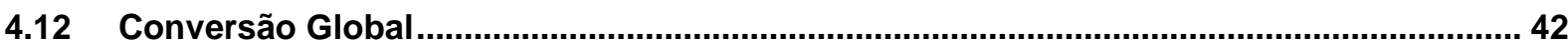

5 MATERIAIS E MÉTODOS........................................................................43

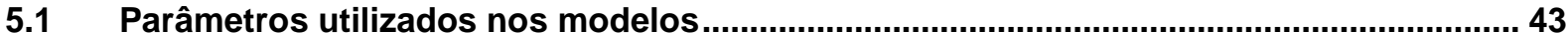

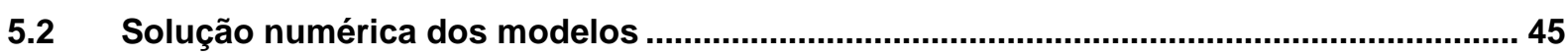

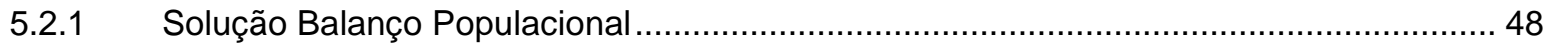

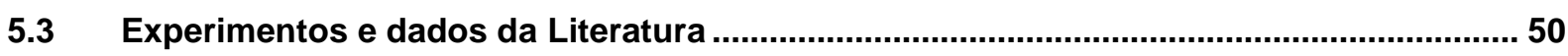

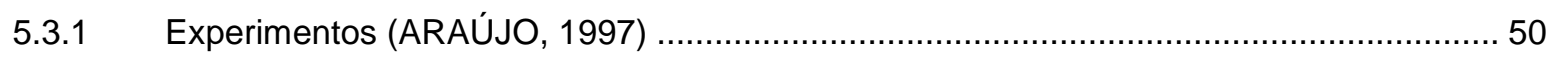

5.3.1.1 Materiais e Condições Operacionais.................................................................. 50

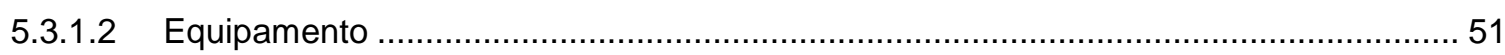




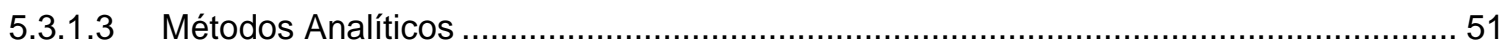

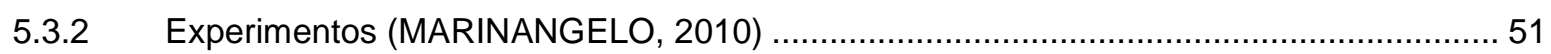

5.3.2.1 Materiais e Condições Operacionais..................................................................... 52

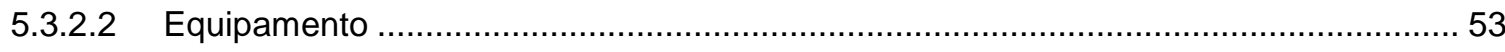

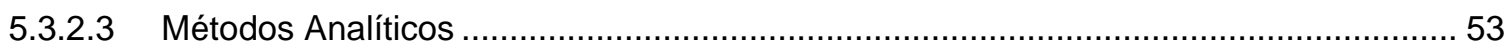

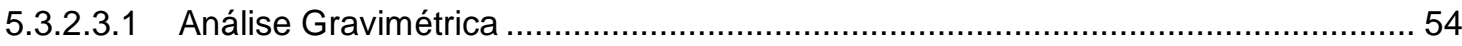

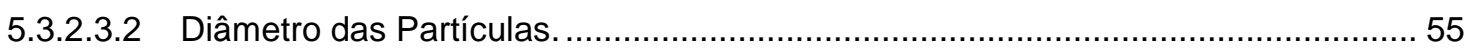

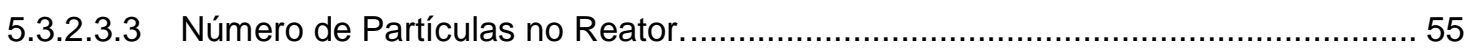

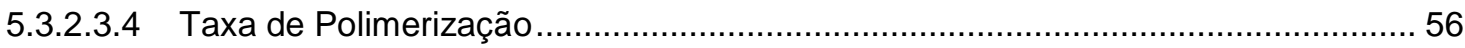

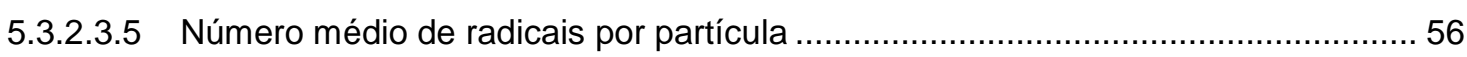

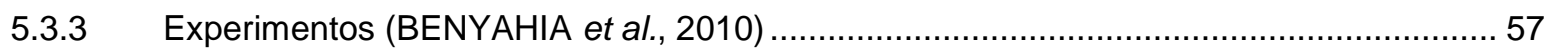

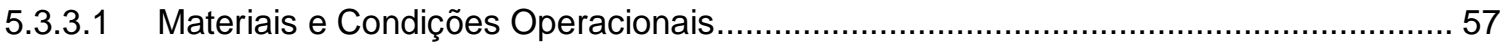

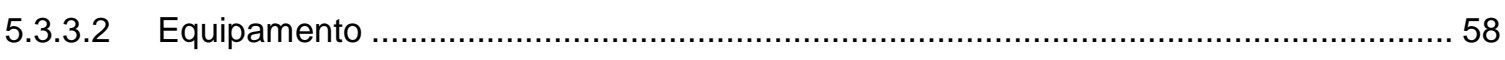

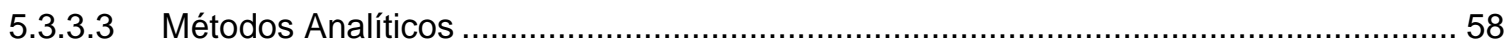

6 RESULTADOS E DISCUSSÃO ................................................................60

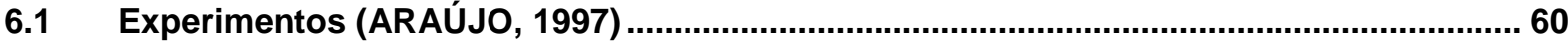

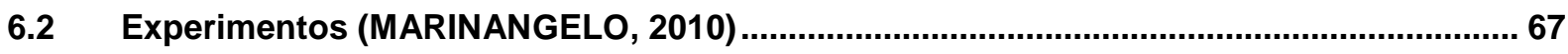

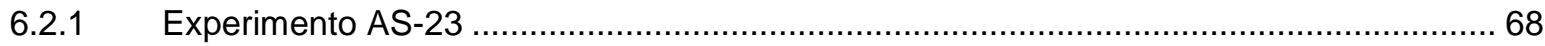

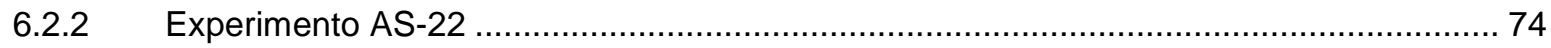

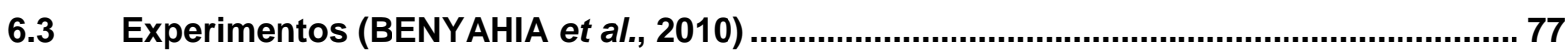

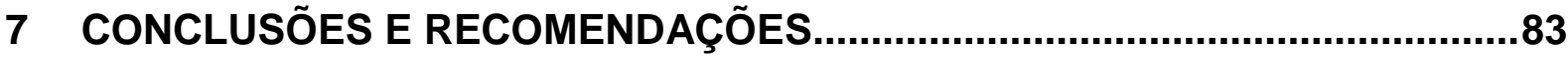

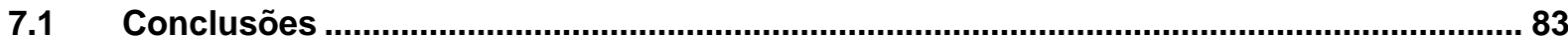

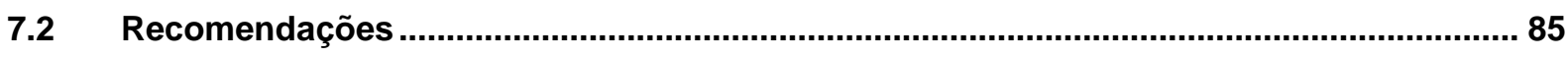

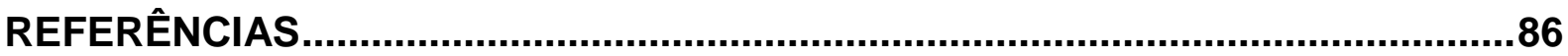

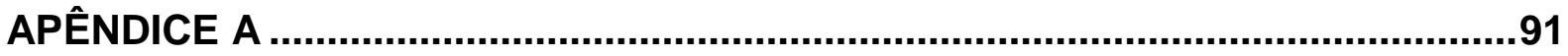

APÊNDICE B ...............................................................................................94

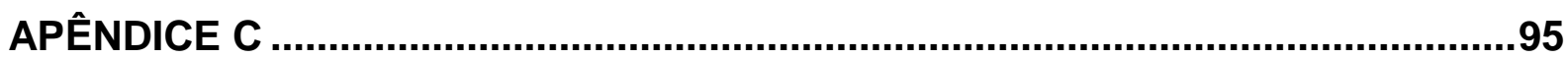




\section{INTRODUÇÃO}

O processo de polimerização em emulsão é usado para a produção de uma grande variedade de produtos incluindo adesivos, tintas, colas, aditivos para papel, aditivos para têxteis, aditivos para materiais de construção, modificadores de impactos para plásticos e em sistema de liberação de fármacos. A variedade de produtos que podem ser produzidas expandiu com o desenvolvimento de técnicas de polimerização em miniemulsão e a implementação de controle de polimerização de radicais no meio disperso (ASUA, 2003).

A estimativa da demanda global de polímeros em emulsão é de um crescimento de 5,4\% por ano considerando 12 milhões de toneladas (base seca) para 2013. Os mercados mais promissores estão pautados em tintas, revestimentos e adesivos. Em países como Brasil, China e Índia, a demanda por polímeros em emulsão deve crescer significativamente com o grande crescimento do mercado de construção nestes países (GRAND VIEW RESEARCH, 2015).

A polimerização em emulsão é reconhecida como um processo heterogêneo complexo, envolvendo transporte de monômeros, radicais livres e de outras espécies entre as fases aquosa e orgânica. Comparando com outros processos heterogêneos de polimerização, como polimerização em suspensão e precipitação, a polimerização em emulsão apresenta-se como o processo mais complexo. A taxa de polimerização na fase orgânica não é controlada somente pela partição de monômero, mas também por outros fenômenos como nucleação de partículas, absorção e dessorção de radicais. A complexidade do sistema aumenta devido às interações que existem entre as fases e os mecanismos de difusão e polimerização dos componentes envolvidos. Ainda, a estabilidade das partículas é afetada pelo tipo , quantidade e força iônica do surfactante no meio disperso (GAO e PENLIDIS, 2002; ARAÚJO e GIUDICI, 2003; ARAÚJO e GIUDICI, 2004).

A modelagem matemática e o seu papel na engenharia são pontos de constante debate, principalmente quando os modelos são implementados em ambientes industriais. Entretanto, os modelos nos auxiliam no entendimento dos fenômenos envolvidos nos processos de interesse e nos ajudam no direcionamento da experimentação. Eles funcionam como uma reserva de conhecimento e podem 
nos auxiliar no entendimento de interações que seriam dificilmente previstas somente com experimentos, principalmente quando muitos fatores estão envolvidos. Os modelos são importantes para o projeto, estimação de parâmetros, análises de sensibilidade, simulação, controle e otimização de processos e ainda evitam os desperdícios de custos com experimentos (DUBÉ et al., 1997).

Os fenômenos considerados no processo de polimerização em emulsão fazem com que a modelagem do sistema seja muito complexa, pois é necessário resolver um conjunto de equações diferenciais não-lineares, acoplada com equações algébricas onde os parâmetros geralmente não são bem conhecidos. A solução numérica deste tipo de modelo passa por um processo de computação intensiva.

Inúmeras contribuições na modelagem do processo de homopolimerização e copolimerização em emulsão foram desenvolvidas, iniciando com o modelo clássico apresentado por Smith-Ewart que identificaram os três principais estágios na polimerização em emulsão: nucleação, crescimento e término da polimerização (HARKINS, 1947). Os mais recentes modelos generalistas desenvolvidos apresentam diversas abordagens para a resolução do problema (DUBÉ et al., 1997; GAO e PENLIDIS, 2002; GINSBURGER et al., 2003; CHERN, 2006).

Os modelos matemáticos mais usados para o processo de polimerização em emulsão consideram como premissa que a distribuição de tamanho de partículas é monodispersa e a massa molar média numérica e mássica são calculadas através do método dos momentos (DUBÉ et al., 1997; GAO e PENLIDIS, 2002; ARAÚJO e GIUDICI, 2003; ARAÚJO e GIUDICI, 2004; BENYAHIA et al., 2010). Outros trabalhos apresentados na literatura resolvem 0 balanço populacional para determinar a distribuições de tamanho de partículas, porém não calculam o massa molar média numérica e mássica do polímero formado (IMMANUEL et al., 2002; MARINANGELO, HIROTA e GIUDICI, 2011).

As propriedades finais da emulsão são diretamente relacionadas com a distribuição de tamanho de partículas (DTP) do látex (IMMANUEL et al., 2002). Os fatores principais que afetam a DTP na polimerização em emulsão são a composição, a concentração e o tipo de surfactante utilizado. Estes fatores também determinam o tamanho médio das partículas, o número total de partículas e a conversão (FEIZ e NAVARCHIAN, 2012). 
A distribuição de massas molares (DMM), microestrutura, temperatura de transição vítrea $\left(T_{g}\right)$ juntamente com a DTP e a morfologia são as principais características que governam fortemente as propriedades dos produtos resultantes e suas aplicações finais. Exemplificando, a distribuição de tamanho de partículas é fortemente correlacionada às propriedades reológicas, de formação de filmes e adesivas dos produtos finais. Contudo, a DMM afeta diretamente as propriedades dos filmes, como elasticidade, resistência mecânica, dureza e resistência a solventes (BENYAHIA et al., 2010).

No capítulo 1 apresenta-se uma introdução ao tema abordado; no capítulo 2 mostra-se os objetivos da presente tese; no capítulo 3 desenvolve-se a revisão bibliográfica considerando o processo de homopolimerização e copolimerização em emulsão; no capítulo 4 descreve-se o modelo matemático desenvolvido a partir do equacionamento sugerido na literatura científica; o capítulo 5 mostra os experimentos realizados por diversos grupos de pesquisa e que serão base para a validação do modelo construído assim como os parâmetros utilizado na modelagem e a estratégia para a solução numérica adotada; o capítulo 6 apresenta e discute os resultados do modelo quando comparados aos dados experimentais; no capítulo 7 apresenta-se a conclusão e as recomendações.

Este trabalho tem como principal contribuição a elaboração de um modelo matemático robusto que tem como principais resultados a distribuição de tamanho de partículas e as massas molares médias numéricas e mássicas ao longo do tempo. Este modelo tem como base as pesquisas e desenvolvimentos realizados ao longo do tempo por diversos de colaboradores no Laboratório de Simulação e Controle de Processos (LSCP) do Departamento de Engenharia Química da Universidade de São Paulo (USP), sendo que o mesmo foi reescrito e evoluído para os resultados de interesse. O código-fonte utilizado está integralmente disponível no Apêndice $\mathrm{C}$ e poderá ser utilizado e evoluído para futuras pesquisas do mesmo grupo de pesquisa ou mesmo por outros grupos que tenham interesse no tema. $O$ modelo poderá também ser adaptado e utilizado na indústria química para prever propriedades finais do látex. 


\section{OBJETIVOS}

O objetivo do presente trabalho foi desenvolver e validar um modelo matemático para simulação da copolimerização em emulsão de acrilato de butila e estireno em reatores batelada e semi-batelada isotérmicos, com o intuito de obter a distribuição de tamanho de partículas (DTP) e a massa molar média numérica e mássica dos polímeros formados. Neste sentido, buscou-se:

- Estudar os fenômenos envolvidos no processo de copolimerização em emulsão e correlacionar com o equacionamento proposto na literatura, entendendo a influência das condições de operação do processo em relação às propriedades finais do látex obtido.

- Construir um modelo matemático para solução do problema e validar com dados da literatura. 


\section{REVISÃO BIBLIOGRÁFICA}

\subsection{Introdução.}

O processo de polimerização em emulsão foi desenvolvido durante a segunda guerra mundial para a produção de borrachas sintéticas de 1-3 butadieno e estireno. Este foi o início da indústria de borrachas sintéticas nos Estados Unidos. Este desenvolvimento teve que ser rápido, devido ao acesso das forças armadas japonesas às fontes de borracha natural, que eram necessárias para o esforço de guerra (ODIAN, 1991).

Os processos de polimerização em emulsão são predominantes para a produção de polímeros de acetato de vinila, cloropreno, copolimerização de acrilatos, copolimerização de butadieno com estireno e acrilonitrilo. Pode ser usado para a produção de metacrilatos, cloreto de vinila, acrilamida e alguns etilenos fluorados (ODIAN, 1991).

A polimerização em emulsão é uma técnica que trabalha com partículas coloidais dispersas em um meio contínuo, geralmente água. Estas dispersões poliméricas são chamadas de látex. As partículas de polímeros são na sua grande maioria esféricas, porém sua morfologia afeta fortemente as propriedades de aplicação final. O diâmetro médio das partículas varia de 50 a 1000 nm, mais comumente entre 80 e $300 \mathrm{~nm}$. Esta faixa de tamanho é uma ordem de grandeza menor que a das partículas obtidas pelo processo de polimerização em suspensão, devido ao mecanismo único para a formação de partículas. O percentual de sólidos em massa é da ordem de 40 a $65 \%$ no látex, sendo que para algumas aplicações um percentual maior é desejado (ASUA, 2007).

Uma das maiores vantagens deste processo, devido a sua natureza multifásica e compartimentada, é a possibilidade de se obter polímeros com alta massa molar a altas taxas de polimerização ao mesmo tempo, produtos com alto teor de sólidos e viscosidade moderada e uma ampla variedade de produtos com alta qualidade, devido às diferentes estratégias de adição de reagente que podem ser escolhidas durante o processo de polimerização. A facilidade de controlar a 
temperatura do reator durante o processo de polimerização também é considerada uma vantagem importante, pois como a polimerização é realizada mais comumente em meio aquoso, a capacidade térmica da água trabalha como uma aliada para esta finalidade. (ARAÚJO e GIUDICI, 2003; ARAÚJO e GIUDICI, 2004; MARINANGELO, HIROTA e GIUDICI, 2011).

O processo também apresenta desvantagens, como a grande necessidade de utilização de aditivos, o que pode afetar a qualidade do produto final, principalmente elevando o grau de impureza. O processo se torna mais caro se o produto final desejado for constituído de polímero seco, pois é preciso fazer a remoção do meio dispersante (MARINANGELO, 2010).

\subsection{Polimerização em emulsão}

O processo de polimerização em emulsão pode ser descrito com base na extensa literatura disponível e através do entendimento dos principais atores envolvidos. Neste tópico explorar-se-á a formulação, os mecanismos e os principais processos de polimerização em emulsão aplicados na indústria química.

\subsubsection{Formulação}

A formulação típica do processo de polimerização em emulsão envolve monômero, água (dispersante), emulsificante, aditivos e iniciador solúvel na fase dispersa. Os monômeros têm baixa solubilidade em água e a razão entre os monômeros duros (alta $T_{g}$ ) e monômeros moles (baixa $T_{g}$ ) são escolhidos para atingir a $T_{g}$ requerida para as aplicações industriais. Monômeros funcionais são utilizados para prover determinadas características especiais como estabilidade e adesão. Os agentes de ligação cruzada (crosslinking) e agentes de transferência de cadeia (CTA) são utilizados para controlar o a estrutura dos polímeros assim como a 
distribuição de massas molares. Uma receita típica do processo de polimerização em emulsão é apresentada na Tabela 3.1 (ASUA, 2007).

Tabela 3.1 - Formulação típica para polimerização em emulsão.

\begin{tabular}{ll}
\hline Monômeros (50-55\% massa) & Agente de Transferência de Cadeia (CTA) \\
Monômeros Principais & Água (45\% em massa) \\
Monômeros Duros (Alta $T_{g}$ ) & Surfactantes (0,5 a 3\% em massa) \\
Estireno & lônicos \\
Metacrilato de Metila & Lauril Sulfato de Sódio \\
Cloreto de Vinila & Não lônicos \\
Acetato de Vinila & Monolaurin $\left[\mathrm{C}_{12} \mathrm{H}_{25}-\left(\mathrm{CH}_{2}-\mathrm{CH}_{2} \mathrm{O}\right)_{n} \mathrm{H}\right]$ \\
Monômeros Moles (Baixa $\left.T_{g}\right)$ & Iniciadores \\
Butadieno & Térmicos \\
Acrilato de Butila & Persulfato de Potássio \\
2-Etil-hexilacrilato & Persulfato de Amônia \\
Veova 10 & Redox \\
Monômeros Secundários & Persulfato de Amônia / $\mathrm{Na}_{2} \mathrm{~S}_{2} \mathrm{O}_{5}$ \\
Ácido Acrílico & \\
Ácido Metacrílico & \\
Acrilamida & \\
Agentes de Crosslink & \\
\hline Fonte: (ASUA, 2007) &
\end{tabular}

Os monômeros são os principais componentes da polimerização e são responsáveis pelas características finais do látex. As reações de homopolimerização, copolimerização e terpolimerização são nomeadas a partir do número de monômeros utilizados. Geralmente são dispersos em água na presença de surfactantes que adsorvem nas gotas de monômero, estabilizando os mesmos.

$\mathrm{Na}$ maioria das formulações, a quantidade de surfactante excede a quantidade necessária para cobrir as gotas de monômero, saturando a fase aquosa e formando micelas que são inchadas com monômeros (ASUA, 2007). Geralmente, usa-se uma mistura de surfactantes iônicos e não-iônicos para garantir a estabilidade do látex formado.

Os iniciadores, geralmente solúveis em água, são usados para gerar os primeiros radicais para o processo de polimerização. A partir dos radicais formados, são gerados radicais livres que atacam os monômeros solubilizados na fase dispersa. Os iniciadores térmicos são usados em processo com alta temperatura 
$\left(75-90^{\circ} \mathrm{C}\right)$ e os iniciadores tipo óxido redução são usados para sistemas com temperatura mais baixa.

O meio dispersante tem papel fundamental na polimerização em emulsão, pois propicia a transferência de monômero entre as partículas as gotas, formação de radicais, decomposição do iniciador e equilíbrio entre as fases e o emulsificante (MARINANGELO, 2010). A grande maioria dos processos de polimerização em emulsão usa a água como meio dispersante, devido as suas propriedades como a baixa solubilidade dos monômeros neste meio, baixa viscosidade e alta capacidade calorifica.

A adição de aditivos é comum nos processos de polimerização em emulsão, sendo que o mais comum é o agente de transferência de cadeia (chain transfer agent - CTA) que tem como principal papel controlar a massa molar média numérica e mássica dos polímeros formados, através da formação de radicais da própria cadeia de CTA.

\subsubsection{Processos}

Os processos industriais de homopolimerização e copolimerização em emulsão são geralmente realizados em reatores semi-batelada. Os processos em batelada são somente utilizados para produzir polímeros com monômeros de reatividades próximas e com baixa taxa de geração de calor; já os reatores contínuos (CSTR) são utilizados para produção em grande escala (ASUA, 2007).

Normalmente em um processo semi-contínuo, o reator é inicialmente carregado com uma fração da formulação (monômeros, emulsificantes, água e iniciador). A carga inicial é então polimerizada em um processo em batelada por algum tempo e então o resto da formulação é adicionada por um período de tempo determinado. Os monômeros podem ser adicionados a partir de uma pré-emulsão ou mesmo puros.

A saturação com nitrogênio antes do início da polimerização é uma prática muito utilizada, pois mitiga a ação do oxigênio como um inibidor. Ainda, os monômeros geralmente são estocados com inibidores e os mesmos são usados sem purificação prévia. 
A Figura 3.1 mostra o macroprocesso para a produção de materiais poliméricos, onde o principal objetivo é a produção de materiais poliméricos com as propriedades finais desejadas para aplicação industrial.

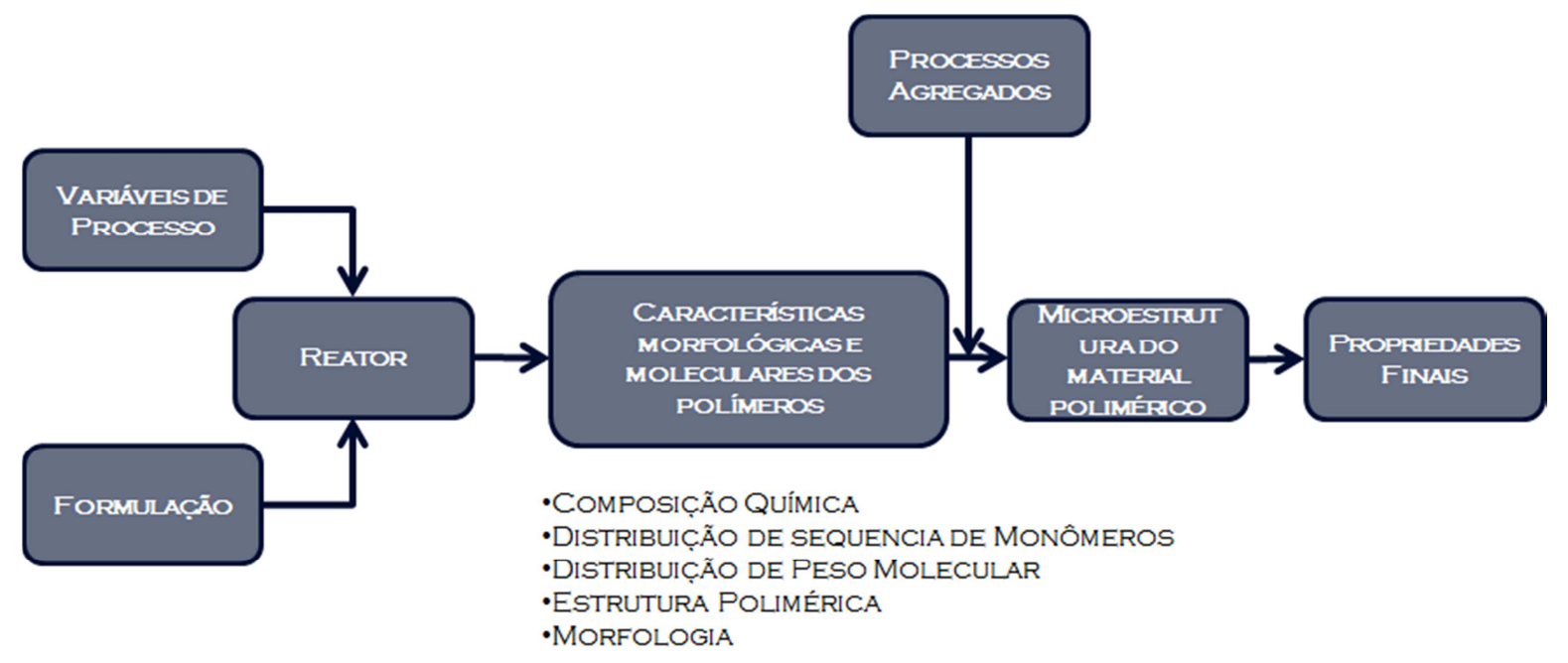

Figura 3.1 - Macroprocesso de produção de materiais poliméricos - Fonte: (ASUA, 2007)

Reatores tubulares de pulso com pratos perfurados foram usados para 0 processo de polimerização em emulsão com vantagens como minimização da variação entre as bateladas, flexibilidade operacional e redução de perdas durante o setup do equipamento. Entretanto, reatores tubulares podem apresentar pontos de incrustações $\mathrm{e}$ até entupimentos com $\mathrm{o}$ aumento da viscosidade do látex (CARVALHO et al., 2006).

\subsubsection{Mecanismos}

Apesar da polimerização em emulsão em reator batelada não ser frequentemente usada na indústria química, ela permite um melhor entendimento dos mecanismos que ocorrem de forma sequencial dentro do reator. Nos processos contínuos e semi-contínuos, os fenômenos ocorrem simultaneamente.

Os surfactantes são absorvidos na superfície das gotas de monômeros dispersos em água, estabilizando as mesmas. Os surfactantes iônicos estabilizam as 
gotas por repulsão eletrostática, enquanto os não-iônicos provêm estabilização estérica (ASUA, 2007).

A polimerização em emulsão começa na fase aquosa, onde os iniciadores hidrofílicos se decompõem e geram os radicais primários. Estes radicais primários propagam na fase aquosa reagindo com os monômeros que estão dissolvidos em água. $O$ crescimento destes radicais na fase aquosa também aumenta a sua hidrofobicidade e estes radicais entram nas micelas. A polimerização em emulsão acontece em três intervalos distintos (GAO e PENLIDIS, 2002):

- Intervalo I: é a primeira fase onde estão presente gotas de monômeros, com tamanho entre $10^{3}-10^{4} \mathrm{~nm}$, micelas e partículas de polímeros. As micelas atuam como uma reserva de monômero. Os radicais que entram nas micelas formam as partículas, com tamanho entre 50-100 nm, que crescem consumindo monômero e para estabilizá-las é necessário mais emulsificante. Devido ao equilíbrio termodinâmico, o monômero presente nas gotas migra para as partículas. Neste intervalo, a concentração de monômero nas partículas se mantém praticamente constante. O Intervalo I termina com o término das micelas.

- Intervalo II: as partículas continuam crescendo e consumindo monômero do sistema. A transferência de monômero das gotas é muito rápida devido ao equilíbrio termodinâmico, mantendo a concentração de monômeros nas partículas constante. O Intervalo II é determinado com o final das gotas de monômero.

- Intervalo III: o monômero da fase aquosa e das micelas é consumido. A concentração de monômero nas partículas e na fase aquosa decresce, devido a polimerização dentro das gotas. A concentração de polímero dentro das partículas aumenta e a reações são controladas pela difusão de radicais dentro das partículas.

As partículas podem ser formadas através da nucleação micelar ou homogênea. Na primeira delas, as partículas são formadas a partir dos radicais que entram nas micelas e se propagam dentro das mesmas. A nucleação homogênea 
ocorre quando os oligômeros formados na fase aquosa se tornam tão hidrofóbicos, devido a sua propagação, que precipitam, formando novas partículas. Os dois mecanismos de nucleação podem ocorrer simultaneamente e a força de cada mecanismo depende das condições operacionais do sistema a ser estudado, sendo a nucleação micelar predominante.

Considerando os fenômenos que ocorrem entre as fases envolvidas na polimerização em emulsão, pode-se destacar na Figura 3.2 os fenômenos que ocorrem na fase aquosa como a propagação e a terminação. As nucleações micelar e homogênea são responsáveis pela formação de partículas, onde ocorrem os fenômenos de propagação, terminação, ramificação e transferência de cadeia. $\mathrm{Na}$ fase partícula, ainda pode ocorrer o fenômeno de coagulação entre partículas. A saída de radicais ilustra o fenômeno da dessorção, sendo que estes radicais podem retornar a fase partícula.

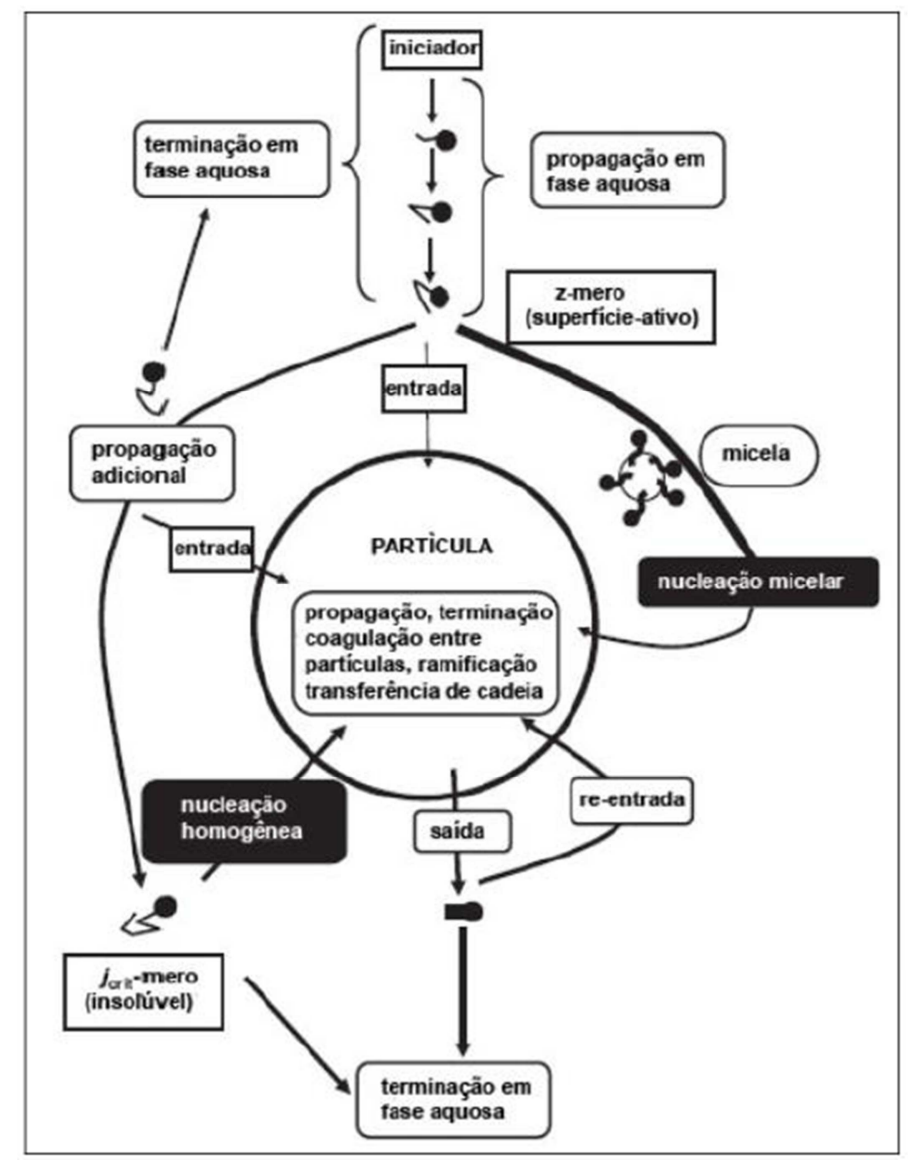

Figura 3.2 - Esquema completo dos fenômenos envolvidos polimerização em emulsão - Fonte:

(THICKETT e GILBERT, 2007) 
A Figura 3.3 mostra os mecanismos envolvidos no processo de polimerização em emulsão. Observa-se que a partir da decomposição do iniciador presente na fase aquosa, inicia-se a formação de radicais que reagem com os monômeros dissolvidos. Os oligômeros formados com seu crescimento vão se tornando cada vez mais hidrofóbicos e podem entrar na fase partícula por difusão ou precipitar formando uma nova partícula. As partículas por sua vez crescem com a propagação dos oligômeros. Mostra-se ainda que radicais pequenos formados dentro das partículas podem dessorver retornando a fase aquosa. Vale ressaltar que as partículas também podem crescer quando há coagulação entre as mesmas, sendo que na polimerização em emulsão a quebra de partículas é um fenômeno raro e pode ser desconsiderado.

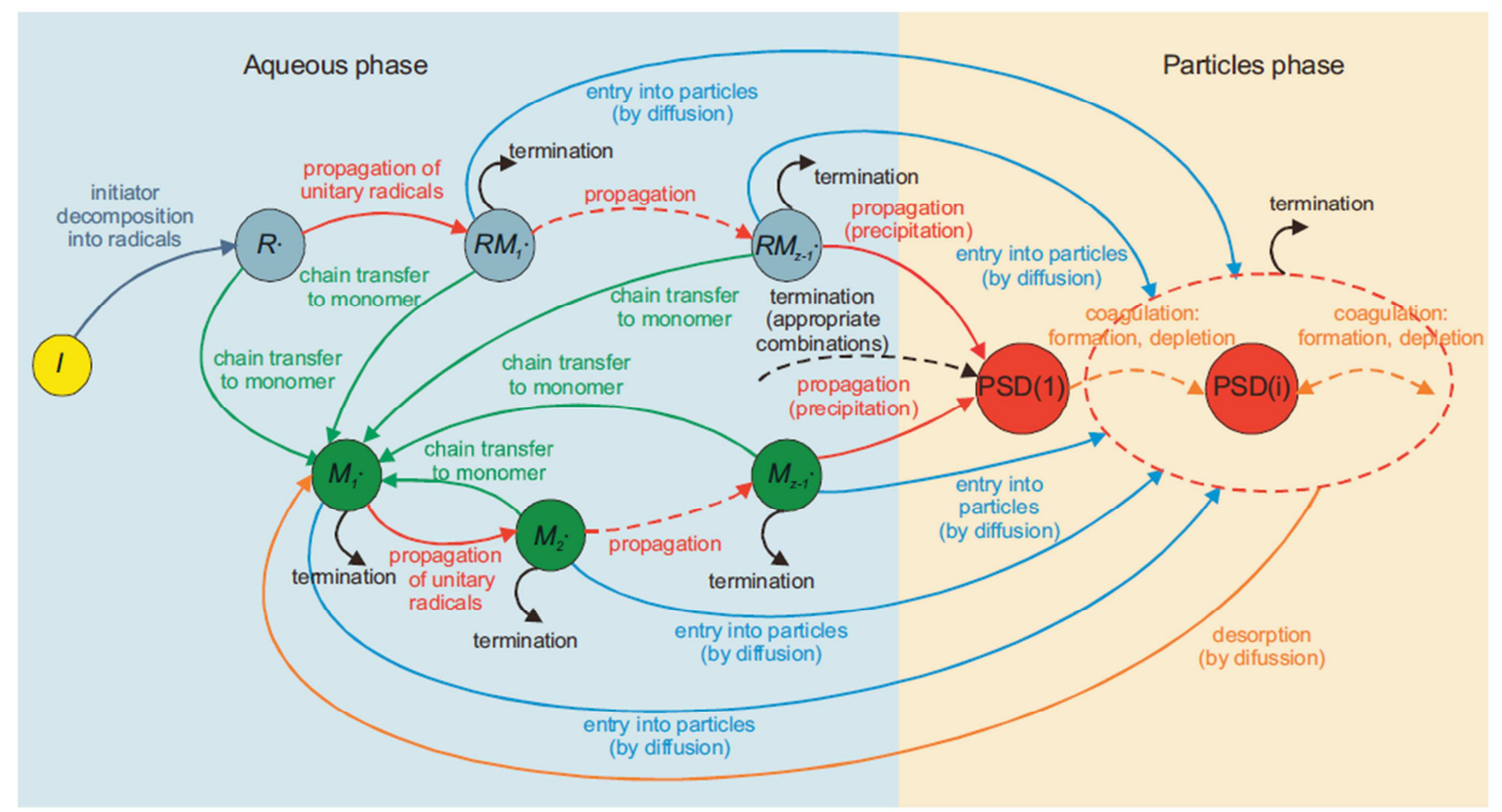

Figura 3.3 - Mecanismo: polimerização em emulsão - Fonte: (HVALA et al., 2011)

Os radicais da fase aquosa crescem até atingirem um comprimento de cadeia mínimo que proporcione um grau hidrofobicidade suficiente para que os mesmos possam entrar em uma micela ou em uma partícula. Os radicais podem crescer na fase aquosa até um tamanho crítico, sem antes entrarem nas micelas ou nas partículas, precipitando e, portanto criando novas partículas.

Vale ressaltar que o número de gotas de monômero é muito menor do que o de partículas de polímero e devido à diferença de área entre estas duas fases, a 
probabilidade de nucleação de gotas é muito pequena e pode ser desconsiderada (ASUA, 2007).

\subsubsection{Distribuição de tamanho de partículas}

A distribuição de tamanho de partículas (DTP) é uma das mais importantes características do látex, determinando suas propriedades reológicas, máximo conteúdo de sólidos, adesão, tempo de secagem, entre outras propriedades. Os látexes com alta concentração de sólidos são um ótimo exemplo para os requerimentos de controle da DTP sendo que a formulação dos mesmos requer uma DTP bem definida para manter níveis aceitáveis de viscosidade do látex (VALE e MCKENNA, 2005). Diversos fatores podem afetar a distribuição de tamanho de partículas em uma polimerização em emulsão.

Como em qualquer sistema de balanço populacional, a DTP é determinada por três grandes fenômenos: nucleação, crescimento e coagulação. Na modelagem de polimerização em emulsão estes fenômenos devem ser considerados, empregando as equações de balanço populacional (IMMANUEL e DOYLE III, 2003; MARINANGELO, 2010).

Inúmeras técnicas são empregadas para a resolução das equações de balanço populacional. As duas mais populares são baseadas no balanço dos momentos e na discretização da equação diferencial que determina a dimensão, transformando a mesma em um sistema de equações diferenciais ordinárias (IMMANUEL et al., 2002; IMMANUEL e DOYLE III, 2003).

A discretização consiste em dividir o domínio em pequenas dimensões e para cada uma das dimensões criadas, o balanço populacional é resolvido considerando a dimensão imediatamente inferior e a imediatamente superior. Todas as equações envolvidas no processo também devem ser resolvidas simultaneamente. Quando maior o número de divisões, maior a precisão do sistema real, porém maior o esforço computacional para resolver o sistema. 


\subsection{Estado da Arte}

As primeiras contribuições em temos de modelagem foram realizadas pelo grupo de Smith e Ewart que apresentaram uma equação empírica que relaciona o número de partículas, a taxa de iniciação e a concentração de emulsificante. Modelos matemáticos apresentados nas décadas de 60 e 70 são evoluções do modelo empírico de Smith e Ewart aplicados a diferentes sistemas monoméricos. Os modelos matemáticos eram passíveis de serem aplicados para sistemas com monômeros com baixa solubilidade em água, sendo que para monômeros mais solúveis apresentavam desvios (GAO e PENLIDIS, 2002).

Os avanços no entendimento da cinética (GILBERT, 1995) da polimerização em emulsão e os avanços na caracterização de polímeros levaram ao melhor entendimento dos fenômenos envolvidos, sendo que inúmeros modelos foram desenvolvidos para descrever diversos sistemas. Pode-se destacar a contribuição de alguns destes modelos na literatura:

- Um modelo detalhado de predição de polimerização em emulsão para reatores batelada e continuo foi desenvolvido para cenários de simulação. Utilizou-se colocação ortogonal em elementos finitos para a solução dos balanços populacionais. A validação experimental e de sensibilidade do modelo foi realizada para um conjunto de cenários (RAWLINGS e RAY, 1988).

- Uma metodologia generalista foi desenvolvida, aplicada à modelagem de polimerização para sistemas multicomponente de radicais livres sendo que os resultados de conversão de monômero, concentração de componentes, massa molar, microestrutura de cadeia, entre outras foram validados a dados experimentais (DUBÉ et al., 1997).

- No intuito de construir um modelo que descrevesse o complexo processo de polimerização em emulsão, uma abordagem generalista foi implementada, 
baseada em uma extensa revisão da literatura cientifica. No modelo, adotouse distribuição de tamanho de partículas monodispersa e o balanço de momentos é utilizado para determinar a massa molar média. Os resultados apresentados foram validados para um conjunto de experimentos (GAO e PENLIDIS, 2002).

- Copolimerização em emulsão de acrilato de butila e estireno foi modelada para reatores tipo batelada e semi-batelada. A determinação dos parâmetros do modelo foi obtida através do ajuste aos resultados de conversão de monômeros, taxa de polimerização e massa molecular média numérica e mássica para o processo em batelada. Adotou-se distribuição de tamanho de partículas monodispersa. Os parâmetros obtidos foram utilizado no modelo em semi-batelada e validação com dados experimentais foi apresentada (GINSBURGER et al., 2003).

- A implementação de modelos para determinação de distribuição de tamanho de partículas em processos de homopolimerização e copolimerização em emulsão foram obtidos através de técnicas de discretização para a solução do balanço populacional (IMMANUEL e DOYLE III, 2003)

- Resultados promissores foram obtidos através técnicas de computação intensiva e modelos tipo black-box como as redes neurais artificiais para descrever o processo de polimerização em emulsão (FERRARI, 2014).

Os modelos matemáticos desenvolvidos para homopolimerização e copolimerização em emulsão adotam premissas e simplificações para viabilizar a solução do conjunto de equações algébrico-diferenciais que descrevem os fenômenos envolvidos. A aplicação do modelo, o conjunto de resultados desejados e as condições operacionais determinam a complexidade do modelo e a abordagem adotada para a solução numérica. 


\section{Modelagem Matemática}

\subsection{Introdução}

Os primeiros estudos considerando a cinética de polimerização em emulsão eram limitados a aspectos como o efeito do iniciador e emulsificante no número de partículas formadas. A taxa de polimerização era tratada de forma qualitativa (GAO e PENLIDIS, 2002).

Os sistemas de polimerização em emulsão são compostos por partículas de diferentes tamanhos, considerando a entrada e saída estocásticas dos radicais com o tempo. Portanto, partículas com diferentes tamanhos têm número de radicais diferentes no seu interior. Na maioria das aplicações, os modelos deste tipo podem se tornar muito complexos, considerando que a taxa de polimerização pode ser estimada e que o sistema é representado por uma população de partículas de tamanho médio (ASUA, 2007). Portanto, pode-se escrever a equação (4.1).

$$
R_{p}=k_{p} \cdot[M]_{p} \cdot \frac{\tilde{n} \cdot N_{p}}{N_{A} \cdot V_{R}}
$$

onde, $N_{p}$ é o número de partículas no reator, $k_{p}$ é a constante da taxa de propagação, $[M]_{p}$ é concentração em monômeros nas partículas, $\tilde{n}$ é o número médio de radicais por partícula, $N_{A}$ é o número de Avogadro e $V_{R}$ é o volume do reator.

Estudos recentes investigam aspectos específicos da polimerização em emulsão. Os recentes avanços na cinética da emulsão e na caracterização dos polímeros propiciou que estudos mais profundos pudessem ser conduzidos tornando possível o melhor entendimento dos fenômenos envolvidos na polimerização em emulsão. A partir destes, pesquisadores evoluíram seus trabalhos para modelagem de homopolimerização, copolimerização e até terpolimerização em emulsão. Inúmeros modelos foram construídos por diferentes grupos de pesquisa e estes 
modelos puderam ser aprimorados para a determinação de cadeias de ramificação longas, tamanho de partículas, sequencia de cadeia e massa molar (GAO e PENLIDIS, 2002).

\subsection{Premissas}

As premissas adotadas para a confecção do modelo proposto de polimerização em emulsão são apresentadas nos tópicos a seguir.

- A distribuição de tamanho de partículas é calculada pelo balanço populacional.

- A nucleação de partículas acontece através dos mecanismos micelar e homogêneo.

- Nucleação Micelar: o radical que atinge um tamanho maior que $z$ na fase aquosa torna-se suficientemente hidrofóbico para entrar nas micelas, produzindo novas partículas ou pode entrar em partículas já formadas.

- Nucleação Homogênea: Os radicais na fase aquosa que atingem um tamanho crítico $j_{\text {crit }}$ precipitam e formam uma nova partícula.

- A propagação de radical ocorre na fase aquosa e dentro das partículas de polímero.

- Novas partículas que nucleiam pelos mecanismos homogêneo ou micelar têm o mesmo raio $r_{n u c}$. Neste modelo foi considerado que $r_{n u c}$ é igual ao raio de micela $r_{\text {mic }}$.

- A coagulação de partículas é desprezada devido à baixa concentração de sólidos no látex.

- As concentrações de monômero nas partículas de polímero, nas gotas de monômero e na fase aquosa estão em equilíbrio termodinâmico e são calculadas utilizando aproximação por coeficiente de partição a cada passo de integração.

- As constantes cinéticas não dependem do tamanho da cadeia do radical e são iguais na fase aquosa e na fase polímero. 
- A reatividade do radical depende somente da última unidade de monômero.

- Pequenos radicais formados pela transferência de cadeia para monômero podem dessorver das partículas de polímero e tem a mesma reatividade e tamanho dos radicais formados pela reação de iniciação.

- Partículas de polímero com o mesmo tamanho têm o mesmo número médio de radicais por partícula.

- A hipótese do estado pseudo-estacionário é adotada para os balanços de radicais na fase aquosa.

- As reações de transferência para polímero e para CTA são consideradas, pois apesar de não afetarem a taxa de polimerização, afetam significantemente a distribuição de massas molares.

- A aproximação pelo método das constantes pseudo-cinéticas foi adotada para calcular as taxas de reação do sistema de copolimerização.

- O efeito gel é considerado na taxa de terminação dos radicais ativos dentro das partículas.

\subsection{Reações}

As reações de radicais livres envolvidas no processo de polimerização são o passo inicial para o desenvolvimento da modelagem. A característica principal da polimerização em emulsão é que as reações ocorrem em fases distintas.

As principais reações químicas envolvidas no processo de polimerização em emulsão podem ser descritas a partir dos fenômenos envolvidos, da sua reação química e da fase em que ocorre, conforme a Tabela 4.1, onde $I$ é o iniciador, $\dot{R}_{i n}$ são radicais provenientes da decomposição de iniciadores, $M_{j}$ é o monômero de tipo $j, \dot{R}_{m, j}$ são os radicais de tamanho $m$ e terminação do tipo $j, D_{m}$ são os polímeros mortos de tamanho $m, A$ é o agente de transferência de cadeia (CTA) e $\dot{A}$ é o radical proveniente do agente de transferência de cadeia. 
Tabela 4.1 - Reações envolvidas no processo de polimerização em emulsão.

\begin{tabular}{l|l|l}
\hline Fenômeno & Reação & Fase \\
\hline Decomposição do Iniciador & $I \stackrel{k_{d}}{\longrightarrow} 2 \dot{R}_{i n}$ & Aquosa \\
\hline Iniciação* & $\dot{R}_{i n}+M_{j} \stackrel{k_{i}}{\longrightarrow} \dot{R}_{1, j}$ & Aquosa \\
\hline Propagação & $\dot{R}_{m, i}+M_{j} \stackrel{k_{p}}{\longrightarrow} \dot{R}_{m+1, j}$ & Aquosa/Polímero \\
\hline Transferência para Monômero & $\dot{R}_{m, i}+M_{j} \stackrel{k_{f m}}{\longrightarrow} D_{m}+\dot{R}_{1, j}$ & Aquosa/Polímero \\
\hline Transferência para Polímero & $\dot{R}_{m, i}+D_{r} \stackrel{k_{f p}}{\longrightarrow} D_{m}+\dot{R}_{r, j}$ & Polímero \\
\hline Transferência para CTA & $\dot{R}_{m, i}+A \stackrel{k_{f A}}{\longrightarrow} D_{m}+\dot{A}$ & Polímero \\
\hline Terminação p/ Combinação & $\dot{R}_{m, i}+\dot{R}_{r, j} \stackrel{k_{c c}}{\longrightarrow} D_{m+r}$ & Aquosa/Polímero \\
\hline Terminação p/ Desproporcionamento & $\dot{R}_{m, i}+\dot{R}_{r, j} \stackrel{k_{t d}}{\longrightarrow} D_{m}+D_{r}$ & Aquosa/Polímero \\
\hline${ }^{*} k_{i} \gg>k_{d}$ & \multicolumn{2}{|l}{}
\end{tabular}

Vale ressaltar que, as reações de transferência de cadeia para polímero e para monômero não afetam significativamente a taxa de polimerização, porém afetam a distribuição de massas molares dos polímeros (MARINANGELO, HIROTA e GIUDICI, 2011). Ainda, considerando a reação de decomposição e iniciação, pode-se afirmar que $k_{i}$ é muito maior que $k_{d}$, sendo o último o limitante. A ordem de grandeza de $k_{i}$ está no mesmo patamar da constante de propagação.

Os fenômenos relacionados à transferência entre fases também são definidos através de reações conforme a Tabela 4.2, onde, Mic. é a micela, Part.é a partícula, $j_{c r i t}$ é o índice que identifica se oligômero sofrerá precipitação gerando uma partícula por nucleação homogênea e $z$ é o índice que determina se o tamanho oligômero da fase aquosa que pode ser capturado pelas micelas ou partículas.

A nucleação heterogênea ocorre quando um radical na fase aquosa de tamanho $m$ e terminação do tipo $i$ migra para a micela, formando assim uma partícula. Este radical tem tamanho $m$ entre $z$ e $j_{c r i t}$. No caso da nucleação homogênea, os radicais de tamanho $j_{c r i t}-1$ podem reagir com o monômero na fase aquosa e precipitar, formando uma nova partícula. A captura de radicais por partículas ocorre quando um radical de tamanho $m$ na fase aquosa entra em uma partícula previamente formada por nucleação heterogênea ou homogênea. $O$ 
fenômeno de dessorção ocorre quando um radical de tamanho unitário dessorve da partícula e retorna a fase aquosa, podendo assim reagir com os monômeros dissolvidos na fase aquosa.

Tabela 4.2 - Fenômenos de transferência de radicais entre a fase aquosa e fase partícula.

\begin{tabular}{l|l|l}
\hline Fenômeno & Reações & Fase \\
\hline Nucleação Heterogênea & {$\left[\dot{R}_{m, i}\right]_{a q}+$ Mic. $\longrightarrow\left[\dot{R}_{m, i}\right]_{a q}+$ Part. } & $\begin{array}{c}\text { Aquosa } \rightarrow \text { Partícula } \\
\left(z<m<j_{c r i t}\right)\end{array}$ \\
\hline Nucleação Homogênea & {$\left[\dot{R}_{j c r i t-1, i}\right]_{a q}+M_{j} \longrightarrow\left[\dot{R}_{\text {crrit }, j}\right]_{p}+$ Part. } & Aquosa $\rightarrow$ Partícula \\
\hline Captura de Radicais & {$\left[\dot{R}_{m, i}\right]_{a q}+$ Part. $\longrightarrow\left[\dot{R}_{m, i}\right]_{p}+$ Part. } & $\begin{array}{c}\text { Aquosa } \rightarrow \text { Partícula } \\
\left(z<m<j_{\text {crit }}\right)\end{array}$ \\
\hline Dessorção & {$\left[\dot{R}_{1, j}\right]_{p} \longrightarrow\left[\dot{R}_{1, j}\right]_{a q}$} & Partícula $\rightarrow$ Aquosa \\
\hline
\end{tabular}

\subsection{Balanço Populacional}

O balanço populacional para o tamanho de partículas na polimerização em emulsão pode ser descrito, considerando os termos de nucleação, crescimento e coagulação. É importante ressaltar que na polimerização em emulsão não há efeito de quebra das partículas, como no caso da cristalização, e o mesmo pode ser desconsiderado. A equação (4.2) representa o balanço populacional:

$$
\frac{\partial F(r, t)}{\partial t}+\frac{\partial}{\partial r}\left(F(r, t) \cdot \frac{d r}{d t}\right)=\Re_{\text {Nuc }} \cdot \delta\left(r-r_{\text {nuc }}\right)+\Re_{\text {Coag }}
$$

onde, $r$ é o raio da partícula não inchada, $F(r, t)$ é a função de densidade probabilística para a distribuição de tamanho de partículas, tal que $F(r, t) \cdot d r$ é a quantidade em mols de partículas com raio entre $r$ e $r+d r, \mathfrak{R}_{\text {Nuc }}$ é a taxa de formação de novas partículas de raio $r_{\text {nuc }}$. A função $\delta\left(r-r_{\text {nuc }}\right)=1$ se $r=r_{\text {nuc }}$, senão 
$\delta\left(r-r_{n u c}\right)=0$. Pode-se calcular a taxa de crescimento das partículas $\left(\frac{d r}{d t}\right)$ através da equação (4.3).

$\frac{d r}{d t}=\frac{1}{4 \pi \cdot r^{2} \cdot \rho_{p o l}} \sum_{i}^{A, B} \sum_{j}^{A, B} k_{p i j} \cdot p_{i} \cdot \frac{\tilde{n}(r, t)}{N_{A}} \cdot\left[M_{j}\right]_{p} \cdot M M_{j}$

onde, $\left[M_{j}\right]_{p}$ é a concentração do monômero $j$ nas partículas de polímero, $k_{p i j}$ é a constante da taxa de propagação entre o radical do tipo $i$ e o monômero do tipo $j$, $N_{A}$ é o número de Avogadro, $M M_{j}$ é a massa molar do monômero do tipo $j, \rho_{p o l}$ é densidade do polímero e $p_{i}$ é a probabilidade do radical do tipo $i$ ser o ativo dentro das partículas. O cálculo desta probabilidade é dado pela equação (4.4).

$$
p_{i}=\frac{k_{p j i} \cdot\left[M_{i}\right]_{p}}{k_{p j i} \cdot\left[M_{i}\right]_{p}+k_{p i j} \cdot\left[M_{j}\right]_{p}} \quad(i, j=A, B \text { e } i \neq j)
$$

A taxa de nucleação pode ser definida como a soma das contribuições dos mecanismos de nucleação micelar e nucleação homogênea.

$$
\mathfrak{R}_{\text {Nuc }}=\mathfrak{R}_{\text {Mic }}+\mathfrak{R}_{\text {Homog }}
$$

onde, $\Re_{\text {Mic }}$ é a taxa de nucleação pelas micelas (heterogênea) $\mathrm{e} \mathfrak{R}_{\text {Homog }}$ é taxa de nucleação homogênea. A taxa de nucleação micelar pode ser descrita através da equação (4.6)

$$
\Re_{M i c}=\sum_{m=z}^{j c r i t-1} \sum_{i}^{A, B} k_{e m i c, i} \cdot p_{a q, i} \cdot\left[\dot{R}_{m}\right]_{a q} \cdot N_{m i c}
$$


onde $k_{e m i c, i}$ é o coeficiente de captura de radicais pelas micelas, $p_{a q, i}$ é a probabilidade do radical de tipo $i$ na fase aquosa entrar na micela, $\left[\dot{R}_{m}\right]_{a q}$ é a concentração de radicais na fase aquosa que pode entrar na micela com $z<m<j$ jrit e $N_{m i c}$ é o número de micelas.

O cálculo de $p_{a q, i}$ é realizado com o mesmo procedimento que o apresentado na equação (4.4), entretanto as concentrações são referentes à fase aquosa. 0 calculo de $k_{e m i c, i}$ é apresentado através da equação (4.7).

$k_{\text {emic }, i}=f_{\text {kemic }} \cdot 4 \cdot \pi \cdot D_{a q, i} \cdot N_{A} \cdot r_{\text {mic }}$

onde, $f_{\text {kemic }}$ é uma constante de eficiência da captura de radicais pelas micelas, $D_{a q, i}$ é a difusividade do monômero $i$ na fase aquosa e $r_{\text {mic }}$ é raio da micela. Pode-se escrever a nucleação homogênea através da equação (4.8).

$\Re_{\text {Homog }}=\left(\sum_{i}^{A, B} \sum_{j}^{A, B} k_{p i j} \cdot p_{a q, i} \cdot\left[M_{j}\right]_{a q}\right) \cdot\left[\dot{R}_{j c r i t-1}\right]_{a q} \cdot V_{a q} \cdot N_{A}$

onde $k_{p i j}$ é a constante de propagação do radical $i$ com o monômero $j,\left[M_{j}\right]_{a q}$ é a concentração de monômero $j$ na fase aquosa e $V_{a q}$ é o volume da fase aquosa no reator.

Usualmente quando a concentração de sólidos na emulsão é baixa, pode-se negligenciar a taxa total de coagulação (IMMANUEL et al., 2002). Entretanto, este termo da modelagem pode ser determinado (IMMANUEL e DOYLE III, 2003).

$\Re_{\text {Coag }}=H\left(r_{\text {sup erior }}-r\right) \cdot \Re_{\text {Formą̧ão }}-H\left(r_{\text {corte }}-r\right) \cdot \Re_{\text {Esgotamento }}$

onde, $H$ é a função de Heaviside (resulta em uma unidade quando o argumento é positivo e zero caso contrário), $r_{\text {corte }}$ é raio mínimo que as partículas podem sofrem coagulação, $r_{\text {Superior }}$ é o raio máximo formado entre partículas menores que 
coagulam, $\Re_{\text {Formasăo }}$ é a taxa de formação de partículas de raio $r$ devido à coagulação e $\Re_{\text {Esgotamento }}$ é taxa de esgotamento de partículas de tamanho $r$ devido à coagulação.

$$
r_{\text {Superior }}=\left(2^{\frac{1}{3}}\right) \cdot r_{\text {corte }}
$$

Os valores de $\Re_{\text {Formaşão }}$ e $\Re_{\text {Essotamento }}$ são apresentados nas equações (4.11) e (4.12).

$\Re_{\text {Formas̆a } a}=\frac{1}{V_{a q}} \int_{r^{\prime}=r_{\text {me }}}^{\frac{r}{2^{1 / 3}}} \beta^{\prime}\left(r^{\prime}, r^{\prime \prime}\right) \cdot F\left(r^{\prime}, t\right) \cdot F\left(r^{\prime \prime}, t\right) \cdot\left\{\frac{r^{2}}{\left[r^{3}-\left(r^{\prime}\right)^{3}\right]^{2 / 3}}\right\} \cdot d r^{\prime}$

$$
\Re_{\text {Esgotamento }}=\frac{1}{V_{a q}} \int_{r_{\operatorname{muc}}}^{r_{\max }} \beta^{\prime}\left(r^{\prime}, r^{\prime \prime}\right) \cdot F\left(r^{\prime}, t\right) \cdot F\left(r^{\prime \prime}, t\right) d r^{\prime}
$$

onde, $\beta^{\prime}\left(r^{\prime}, r^{\prime \prime}\right)$ é a taxa de coagulação intrínseca dependente entre partículas de raio $r^{\prime}$ e $r^{\prime \prime}$, que depende da concentração de surfactante e, portanto é variável no tempo. Este parâmetro depende também dos potenciais de atração e repulsão agindo entre as partículas. O potencial de atração é atribuído à força de Van der Walls e o de repulsão depende da quantidade de emulsificante colocado na receita para a polimerização em emulsão (IMMANUEL e DOYLE III, 2003).

Neste modelo, a coagulação de partículas não foi considerada e portanto $\Re_{\text {Coag }}=0$ devido ao baixo teor de sólidos no reator e a falta de informações do sistema utilizado, necessárias para determinar os parâmetros deste termo da modelagem. 


\subsection{Número médio de radicais nas partículas}

O número médio de radicais por partícula pode também ser modelado através de um balanço populacional, considerando as taxas de absorção, dessorção e terminação. Este método apresenta resultados acurados para um amplo conjunto de condições operacionais (LI e BROOKS, 1993).

$$
\frac{d \tilde{n}(r, t)}{d t}=\sigma-k_{d e s} \cdot \tilde{n}(r, t)-\frac{c \cdot k_{t}}{v_{p}(r) \cdot N_{A}} \cdot \tilde{n}(r, t)^{2}
$$

onde, $\sigma$ é a taxa de absorção de radicais nas partículas, $k_{\text {des }}$ é a constante de dessorção, $k_{t}$ é a constante média de terminação na fase partícula, $v_{p}(r)$ é o volume de uma partícula com raio $r$ e $c$ é um coeficiente calculado.

A taxa de absorção dos radicais pelas partículas pode ser calculada através da equação (4.14).

$$
\sigma=k_{e}(r) \cdot\left[\dot{R}_{m}\right]_{a q} \quad(z<m<j c r i t)
$$

Diversos métodos podem ser encontrados na literatura para o cálculo da constante de dessorção, porém os mesmos apresentam pequena diferença entre seus resultados (DUBÉ et al., 1997). A dessorção ocorre quando um radical pequeno migra da partícula para a fase aquosa. Assume-se que estes radicais tem tamanho de uma unidade monomérica e a sua formação depende da transferência de cadeia para monômero e para polímero, sendo que o último fenômeno é considerado desprezível para modelagem. Vale ressaltar que o radical advindo de dessorção pode reagir na fase aquosa por propagação ou terminação. O conjunto de equações (4.15), (4.16) e (4.17) mostra a taxa global de dessorção (FORCADA e ASUA, 1990). 
$k_{\text {des }}=\sum_{i}^{A, B} k_{d e s}^{i}$

$k_{d e s}^{i}=\left(k_{f m A i} \cdot p_{A}+k_{f m B i} \cdot p_{B}\right) \cdot\left[M_{i}\right]_{p} \cdot\left[\frac{k_{o i}}{\beta_{i} \cdot k_{o i}+k_{p i A} \cdot\left[M_{A}\right]_{p}+k_{p i B} \cdot\left[M_{B}\right]_{p}}\right] \quad(i=A, B)$

onde, $k_{d e s}^{i}$ é o coeficiente global de dessorção de radicais do tipo $i$ para a fase aquosa, $k_{f m j i}$ é a constante da taxa de transferência de monômero entre monômero $j$ e radicais de tipo $i,\left[M_{i}\right]_{p}$ é concentração e monômero $i$ nas partículas, $k_{o i}$ é a constante da taxa de dessorção de radicais de radicais do tipo $i$ e $\beta_{i}$ probabilidade de um radical monomérico do tipo $i$ reagir na fase aquosa por propagação ou terminação.

$k_{o i}=\frac{12 \cdot D_{a q, i}}{K_{p}^{i} \cdot\left(2 \cdot r_{s}\right)^{2}} \cdot\left(1+\frac{2 \cdot D_{a q, i}}{K_{p}^{i} \cdot D_{p, i}}\right)^{-1}$

sendo que $D_{p, i}$ é a difusividade do monômero $i$ na fase partícula, $r_{s}$ é o raio das partículas inchadas, $K_{p}^{i}$ é o coeficiente e partição do monômero $i$ entre as partículas e a fase aquosa.

A equação (4.18) mostra a probabilidade de um radical do tipo $i$ reagir na fase aquosa por propagação ou terminação (CASELLA, ARAUJO e GIUDICI, 2003).

$$
\beta_{i}=\frac{\left(k_{p i A} \cdot\left[M_{A}\right]_{a q}+k_{p i B} \cdot\left[M_{B}\right]_{a q}\right)+\left(k_{t i A} \cdot p_{A, a q}+k_{t i B} \cdot p_{B, a q}\right) \cdot\left[\dot{R}_{T}\right]_{a q}}{\left(k_{p i A} \cdot\left[M_{A}\right]_{a q}+k_{p i B} \cdot\left[M_{B}\right]_{a q}\right)+\left(k_{t i A} \cdot p_{A, a q}+k_{t i B} \cdot p_{B, a q}\right) \cdot\left[\dot{R}_{T}\right]_{a q}+\frac{k_{e}}{V_{R}} \cdot \int_{r_{n u c}}^{r_{\max }} \cdot F(r, t) \cdot d r}
$$


onde, $k_{t i j}$ é a constante de terminação entre radicais com terminação tipo $i$ e tipo do $j$. A constante $c$ depende dos valores de $\sigma, k_{\text {des }}$ e $k_{t}$ pode ser calculada através da equação (4.19).

$$
c=\frac{2 \cdot\left(2 \cdot \sigma+k_{d e s}\right)}{2 \cdot \sigma+k_{d e s}+\frac{k_{t}}{v_{p}(r) \cdot N_{A}}}
$$

O coeficiente de entrada de radicais nas partículas de raio inchado $r_{s}$ pode ser calculado através da equação (4.20).

$$
k_{e}\left(r_{s}\right)=f_{k e} \cdot 4 \cdot \pi \cdot N_{A} \cdot r_{s} \cdot\left(D_{a q, A} \cdot p_{A, a q}+D_{a q, B} \cdot p_{B, a q}\right)
$$

onde, $f_{k e}$ é uma constante de eficiência da captura de radicais pelas partículas e $r_{s}$ é o raio das partículas inchadas com monômero. Para determinação de $r_{s}$, pode-se utilizar a equação (4.21) (IMMANUEL et al., 2002).

$$
r_{s}^{3}=\frac{r^{3}}{1-\sum_{j}^{A, B} \frac{M M_{j} \cdot\left[M_{j}\right]_{p}}{\rho_{j}}}
$$

onde, $\rho_{j}$ é a densidade do monômero $j$. A constante de terminação média na fase partícula pode ser calculada a partir das constantes de terminação e do coeficiente do efeito gel.

$$
k_{t}=g \cdot \sum_{i}^{A, B} \sum_{j}^{A, B} p_{i} \cdot p_{j} \cdot k_{t i j}
$$


onde, $k_{t i j}$ é a constante da taxa de terminação entre radicais do tipo $i$ e os radicais do tipo $j$. A constante g pode ser calculada através da equação (4.23) e (4.24) (FRIIS e HAMIELEC, 1976; PLESSIS et al., 2001).

$g=\exp \left[g_{A} Y_{B A}+g_{B}\left(1-Y_{B A}\right)\right]$

$g_{i}=-m_{i} \cdot\left[B_{i} \cdot \boldsymbol{\phi}_{p}^{p}+C_{i} \cdot\left(\phi_{p}^{p}\right)^{2}+D_{i} \cdot\left(\phi_{p}^{p}\right)^{3}\right]$

onde $Y_{B A}$ é composição de polímero em relação ao monômero $A$, $\phi_{p}^{p}$ é a fração volumétrica de polímero nas partículas, $m_{i}, B_{i}, C_{i}$ e $D_{i}$ são constantes que dependem do monômero $i$.

\subsection{Radicais na fase aquosa}

A primeira etapa da polimerização, fase de iniciação, envolve a formação de radicais livres extremamente reativos. As reações de polimerização em massa, suspensão ou em solução envolvem iniciadores orgânicos, que são decompostos em radicais livres através do aumento de temperatura ou por reação fotoquímica. No caso da polimerização em emulsão, há dois métodos para iniciação, sendo que o mais comum deles é o da decomposição do iniciador por reação tipo óxido-redução (redox), que pode ser utilizada para reações a baixas temperaturas, ou decomposição térmica quando o processo pode ocorrer em altas temperaturas, sendo esta menos usual (DUBÉ et al., 1997; GAO e PENLIDIS, 2002).

Considerando a iniciação através de reações tipo redox, o cálculo de radicais na fase aquosa é feito através da hipótese do estado pseudo-estacionário. O radical de tamanho $j_{\text {crit }}$ define o tamanho que os radicais passam a ser insolúveis na fase aquosa e geram uma nova partícula de polímero. Este tamanho crítico depende da solubilidade do monômero na fase aquosa e da temperatura do meio. 
O radical de tamanho $z$ é o menor radical que pode entrar em uma micela ou em uma partícula de polímero (ARAÚJO e GIUDICI, 2003; CASELLA, ARAUJO e GIUDICI, 2003; ARAÚJO e GIUDICI, 2004; MARINANGELO, HIROTA e GIUDICI, 2011).

$\left[\dot{R}_{T}\right]_{a q}=\sum_{n=1}^{j c r i t-1}\left[\dot{R}_{n}\right]_{a q}$

onde, $\left[\dot{R}_{T}\right]_{a q}$ é a concentração de radicais livres totais na fase aquosa e $\left[\dot{R}_{n}\right]_{a q}$ concentração de radicais de tamanho $n$ na fase aquosa.

$\left[\dot{R}_{1}\right]_{a q}=\frac{2 \cdot f \cdot k_{d} \cdot[I]+\frac{k_{d e s}}{V_{R}} \cdot\left[\int_{r_{\text {ruc }}}^{r_{\max }} \tilde{n}(r, t) \cdot F(r, t) \cdot d r\right]}{\sum_{j}^{A, B} k_{p j, a q} \cdot\left[M_{j}\right]_{a q}+k_{t, a q} \cdot\left[\dot{R}_{T}\right]_{a q}}$.

$(n=2,3, \ldots, z-1):$

$\left[\dot{R}_{n}\right]_{a q}=\frac{\left[\dot{R}_{n-1}\right]_{a q} \cdot \sum_{j}^{A, B} k_{p j, a q} \cdot\left[M_{j}\right]_{a q}}{\sum_{j}^{A, B} k_{p j, a q} \cdot\left[M_{j}\right]_{a q}+k_{t, a q} \cdot\left[\dot{R}_{T}\right]_{a q}}$

$\alpha=\frac{\sum_{j}^{A, B} k_{p j, a q} \cdot\left[M_{j}\right]_{a q}}{\sum_{j}^{A, B} k_{p j, a q} \cdot\left[M_{j}\right]_{a q}+k_{t, a q} \cdot\left[\dot{R}_{T}\right]_{a q}}$

$\left[\dot{R}_{n}\right]_{a q}=\left[\dot{R}_{n-1}\right]_{a q} \cdot \alpha$

$\left(n=z, \ldots, j_{\text {crit }}-1\right)$ : 


$$
\left[\dot{R}_{n}\right]_{a q}=\frac{\left[\dot{R}_{n-1}\right]_{a q} \cdot \sum_{j}^{A, B} k_{p j, a q} \cdot\left[M_{j}\right]_{a q}}{\sum_{j}^{A, B} k_{p j, a q} \cdot\left[M_{j}\right]_{a q}+k_{t, a q} \cdot\left[\dot{R}_{T}\right]_{a q}+\left(\frac{k_{e} \cdot N_{A} \cdot \int_{r_{n u c}}^{r_{\max }} F(r, t) \cdot d r+k_{\text {emic }} \cdot N_{m i c}}{V_{R} \cdot N_{A}}\right)}
$$

$$
\beta=\frac{\sum_{j}^{A, B} k_{p j, a q} \cdot\left[M_{j}\right]_{a q}}{\sum_{j}^{A, B} k_{p j, a q} \cdot\left[M_{j}\right]_{a q}+k_{t, a q} \cdot\left[\dot{R}_{T}\right]_{a q}+\left(\frac{k_{e} \cdot N_{A} \cdot \int_{r_{\text {muc }}}^{r_{\max }} F(r, t) \cdot d r+k_{\text {emic }} \cdot N_{\text {mic }}}{V_{R} \cdot N_{A}}\right)}
$$

$$
\left[\dot{R}_{n}\right]_{a q}=\left[\dot{R}_{n-1}\right]_{a q} \cdot \beta
$$

onde, $f$ é o fator que determina a eficiência do iniciador, $[I]$ é a concentração de iniciador, $k_{d}$ é a constante de decomposição do iniciador e $k_{t, a q}$ é a constante da taxa de terminação de polímeros na fase aquosa. Os valores de $k_{e}$ e $k_{\text {emic }}$ provém das equações (4.20) e (4.7) respectivamente.

A partir do equacionamento apresentado, pode-se mostrar que o número de radicais totais na fase aquosa depende de $\left[\dot{R}_{1}\right]_{a q}, \alpha$ e $\beta$.

$$
\begin{aligned}
& {\left[\dot{R}_{n}\right]_{a q}=\left[\dot{R}_{1}\right]_{a q} \cdot \alpha^{z-2} \cdot \beta^{n-(z-1)}} \\
& {\left[\dot{R}_{j_{c r i t}-1}\right]_{a q}=\left[\dot{R}_{1}\right]_{a q} \cdot \alpha^{z-2} \cdot \beta^{j_{c r i t}-z}} \\
& {\left[\dot{R}_{T}\right]_{a q}=\left[\dot{R}_{1}\right]_{a q} \cdot\left[1+\sum_{n=2}^{z-1} \alpha^{n-1}+\alpha^{z-2} \cdot \sum_{n=z}^{j_{c r i t-1}} \beta^{n-(z-1)}\right]}
\end{aligned}
$$


$\left[\dot{R}_{m}\right]_{a q}=\left[\dot{R}_{1}\right]_{a q} \cdot \alpha^{z-2} \cdot \sum_{n=z}^{j_{c r i t}-1} \beta^{n-(z-1)}$

onde, $\left[\dot{R}_{m}\right]_{a q}$ é a concentração de radicais na fase aquosa que podem entrar nas micelas ou partículas.

Vale ressaltar que o equacionamento apresentado é geral para casos onde $z>2$. Entretanto, para casos onde $z=1$ ou $z=2$ pode-se reescrever as equações para o cálculo das concentrações de radicas na fase aquosa conforme apresentado no Apêndice A.

A obtenção dos valores de $\left[\dot{R}_{1}\right]_{a q}$ e $\left[\dot{R}_{T}\right]_{a q}$ podem ser obtidos através de um procedimento numérico iterativo para a solução do sistema de equações não lineares.

\subsection{Partição de Monômero}

A complexidade do processo de polimerização em emulsão também vem do fato que todos os ingredientes da receita estão presentes em todas as fases. Acompanhar a concentração de todos os componentes em cada fase não é uma tarefa fácil, pois não há teoria satisfatória que possa descrever de forma robusta o fenômeno da partição. Há um agravamento desta complexidade quando os processos são de copolimerização ou terpolimerização, onde diversos monômeros e os polímeros coexistem na mesma fase. A maioria dos grupos de pesquisa busca resolver o problema da partição através de correlações empíricas, o que implica em realização de experimentos. Os coeficientes de partição são facilmente aplicados aos modelos, porém quando o número de monômeros envolvidos aumenta este método se torna intensivo e incerto. Ainda, há a necessidade de se prever a partição de monômeros para uma nova emulsão sem precisar da medida dos coeficientes de partição (DUBÉ et al., 1997; GAO e PENLIDIS, 2002).

A abordagem teórica tem algumas vantagens sobre a abordagem empírica em alguns casos. O princípio por trás desta abordagem mostra que o potencial 
químico de cada espécie é igual para todas as fases envolvidas sobre a premissa que o equilíbrio químico pode se estabelecer rapidamente sem limitação de transferência de massa. Entretanto, o modelo teórico mostra-se mais difícil para resolver devido às equações não lineares presentes em adição à dificuldade de se obter parâmetros para o modelo (GAO e PENLIDIS, 2002).

Abordagem empírica sugere que a concentração de cada componente em cada uma das fases está em equilíbrio termodinâmico e as suas concentrações são calculadas pelos coeficientes de partição.

$K_{d}^{j}=\frac{V_{d}^{j} / V_{d}}{V_{a q}^{j} / V_{a q}}$

$(j=A, B)$

$$
K_{p}^{j}=\frac{V_{p}^{j} / V_{p}}{V_{a q}^{j} / V_{a q}}
$$$$
(j=A, B)
$$

onde $V_{d}^{j}$ é volume de monômero $j$ nas gotas, $V_{d}$ é o volume das gotas, $V_{p}^{j}$ é o volume de monômero $j$ nas partículas, $V_{p}$ é o volume das partículas, $V_{a q}^{j}$ é 0 volume de monômero $j$ na fase aquosa, $V_{a q}$ é volume total da fase aquosa, $K_{p}^{j}$ é o coeficiente e partição do monômero $j$ entre as partículas e a fase aquosa e $K_{d}^{j}$ é o coeficiente e partição do monômero $j$ entre as gotas e a fase aquosa.

Os balanços volumétricos para cada uma das fases presentes, partículas, gotas e fase aquosa pode ser descrito.

$V_{a q}=V_{H_{2} O}+V_{a q}^{A}+V_{a q}^{B}$

$V_{p}=V_{p o l}+V_{p}^{A}+V_{p}^{B}$ 
$V_{d}=V_{d}^{A}+V_{d}^{B}$

onde, $V_{H_{2}} \mathrm{O}$ é o volume de água no reator e $V_{p o l}$ é o volume de polímero no reator. $\mathrm{O}$ balanço de volume de cada um dos monômeros distribuído entre as fases pode ser descrito conforme a equação (4.42). Adota-se a hipótese de solução ideal para este caso onde $\Delta V=0$ e o volume de CTA pode ser desprezado.

$$
\begin{aligned}
& V^{j}=V_{d}^{j}+V_{p}^{j}+V_{a q}^{j} \quad \quad(j=A, B) \\
& V_{R}=V_{d}+V_{a q}+V_{p}
\end{aligned}
$$

A cada instante da reação, os valores de $V_{R}, V_{\mathrm{H}_{2} \mathrm{O}}, V_{p o l}, V^{A}$ e $V^{B}$ devem ser calculados utilizando as equações (4.44), (4.45) e (4.46).

$$
V_{\mathrm{H}_{2} \mathrm{O}}=V_{R} \cdot \varphi
$$

$$
V_{p o l}=\sum_{j}^{A, B}\left(\left[M_{j 0}\right]-\left[M_{j}\right]\right) \cdot \frac{V_{R} \cdot M M_{j}}{\rho_{p o l}}
$$

$$
V^{j}=\frac{\left[M_{j}\right] \cdot V_{R} \cdot M M_{j}}{\rho_{j}} \quad(j=A, B)
$$

onde, $\varphi$ é a fração volumétrica de água, $\left[M_{j 0}\right]$ é a concentração acumulada de monômero $j$ alimentado no reator e $\rho_{p o l}$ é a densidade do polímero formado. As concentrações de monômero na fase partícula e na fase aquosa podem ser calculadas pelas equações (4.47) e (4.48) respectivamente. 
$\left[M_{j}\right]_{p}=\frac{V_{p}^{j} \cdot \rho_{j}}{V_{p} \cdot M M_{j}}$

$(j=A, B)$

$\left[M_{j}\right]_{a q}=\frac{V_{a q}^{j} \cdot \rho_{j}}{V_{a q} \cdot M M_{j}}$

$(j=A, B)$

As equações acima apresentadas não consideram o volume do agente de transferência de cadeia (CTA). Entretanto, a difusão do CTA das gotas para a fase aquosa é um passo limitante do processo de polimerização em emulsão (BENYAHIA et al., 2010) e será tratada no tópico 4.9. O Apêndice B mostra o algoritmo interativo para a solução da partição de monômeros.

\subsection{Partição de Surfactante}

O emulsificante pode estar presente na fase aquosa livre ou como uma micela e também pode estar adsorvido na superfície das partículas de polímero ou nas gotas de monômero. Apesar das gotas de monômero serem maiores que as partículas de polímero, o número de gotas é muito menor, consequentemente, possuem uma área total muito menor que área total das partículas poliméricas. Portanto, pode-se desprezar a quantidade de emulsificante adsorvido nas gotas de monômero (ARAÚJO e GIUDICI, 2003; ARAÚJO e GIUDICI, 2004).

$V_{R} \cdot[S]=V_{a q} \cdot[S]_{a q}+N_{m i c} \cdot \gamma+A_{p T} \cdot[S]_{a d s}$

$\gamma=\frac{4 \cdot \pi \cdot r_{m i c}^{2}}{a_{s}}$ 
onde $A_{p T}$ representa a área total das partículas, $[S]$ é a concentração de emulsificante no reator, $[S]_{a q}$ é a concentração de emulsificante na fase aquosa, $[S]_{a d s}$ é a concentração de emulsificante adsorvido nas micelas por unidade área e $a_{s}$ é a área que um mol de emulsificante recobre das partículas.

$$
A_{p T}=\int_{r_{m u c}}^{r_{\max }} 4 \cdot \pi \cdot r_{s} \cdot N_{A} \cdot F(r, t) \cdot d r
$$

Portanto, caso a concentração de emulsificantes na fase aquosa seja maior que a concentração micelar crítica CMC, pode-se reescrever a equação (4.49) e isolar o termo que determina o número de micelas. Caso contrário, o número de micelas é igual a zero.

$$
\begin{aligned}
& V_{R} \cdot[S]=V_{a q} \cdot C M C+N_{m i c} \cdot \gamma+A_{p T} \cdot[S]_{a d s} \\
& {[S]_{a d s}=\frac{1}{a_{s}}} \\
& N_{m i c}=\frac{V_{R} \cdot[S]-C M C \cdot V_{a q}-\frac{A_{p T}}{a_{s}}}{\gamma}
\end{aligned}
$$

\subsection{Partição de Agente de Transferência de Cadeia.}

A difusão de CTA das gotas para a fase aquosa é o limitante para a distribuição de CTA entre as fases, consequentemente a concentração nas partículas fica abaixo da concentração de saturação. As equações (4.55) e (4.56) 
podem ser escritas para calcular a concentração de CTA dentro das partículas (BENYAHIA et al., 2010).

$$
[A]_{p}=\frac{[A]_{p}^{e}}{1+\frac{\sum_{j}^{A, B} k_{f A j} \cdot p_{j} \int_{r_{n u c}}^{r_{\max }} \tilde{n}(r, t) \cdot F(r, t) \cdot d r}{k_{f A, a q} \cdot A_{d} \cdot K_{p A}}}
$$

$$
[A]_{p}^{e}=\frac{[A] \cdot V_{R} \cdot V_{p}}{V_{p}+V_{d} \cdot \theta+V_{a q} \cdot K_{p A}}
$$

onde, $[A]_{p}$ é a concentração de CTA dentro das partículas, $A_{d}$ é a área das gotas, $K_{p A}$ é constante de partição do CTA entre a fase aquosa e as partículas, $[A]_{p}^{e}$ é a concentração de partículas em equilíbrio termodinâmico e $\theta$ é coeficiente relacionado ao grau de saturação das partículas.

\subsection{Balanço Material}

O balanço material do processo de polimerização em emulsão pode ser descrito considerando cada um dos componentes. As reações químicas apresentadas na Tabela 4.1 são a fonte principal para a construção do sistema de equações diferenciais apresentadas nos tópicos abaixo.

\subsubsection{Monômeros}

Os balanços de monômeros do tipo $i$ presentes no reator e o total número de monômeros $i$ introduzidos no reator podem ser descritos pelas equações (4.57) e (4.58) respectivamente (MARINANGELO, HIROTA e GIUDICI, 2011). 
$\frac{d\left(\left[M_{i}\right] \cdot V_{R}\right)}{d t}=-\Re_{p i}-\Re_{f i}+Q_{f} \cdot\left[M_{i, f}\right]$

$\frac{d\left(\left[M_{i 0}\right] \cdot V_{R}\right)}{d t}=Q_{f} \cdot\left[M_{i, f}\right]$

$\Re_{p i}=\sum_{j}^{A, B} k_{p j i, a q} \cdot p_{j, a q} \cdot\left[\dot{R}_{T}\right]_{a q} \cdot V_{a q} \cdot\left[M_{i}\right]_{a q}+\sum_{j}^{A, B} k_{p j i} \cdot p_{j} \cdot\left[M_{i}\right]_{p} \cdot \int_{r_{n u c}}^{r_{\max }} \tilde{n}(r, t) \cdot F(r, t) \cdot d r$

$\mathfrak{R}_{f i}=\sum_{j}^{A, B} k_{f n j i} \cdot p_{j} \cdot\left[M_{i}\right]_{p} \cdot \int_{r_{n u c}}^{r_{\max }} \tilde{n}(r, t) \cdot F(r, t) \cdot d r$

$k_{p i}=\sum_{j}^{A, B} k_{p j i} \cdot p_{j}$

onde $k_{p i}$ é a constante da taxa de propagação para o monômero $i$. Vale ressaltar que a taxa de propagação cruzada $k_{p j i}$ relaciona os monômeros $A$ e $B$ com as taxas de propagação através da razão de reatividade $r_{i j}$ que pode ser calculada através da equação (4.62).

$r_{i j}=\frac{k_{p i i}}{k_{p_{i j}}}$

$$
(i \neq j)
$$




\subsubsection{Iniciador}

A equação de balanço para o iniciador é escrita na forma:

$\frac{d\left([I] \cdot V_{R} \cdot \varphi\right)}{d t}=-k_{d} \cdot[I] \cdot V_{R} \cdot \varphi+Q_{f} \cdot\left[I_{f}\right]$

onde, $\left[I_{f}\right]$ é a concentração de iniciador na corrente de entrada e $k_{d}$ é a constante da taxa de decomposição do iniciador em meio aquoso.

\subsubsection{Emulsificante}

O balanço para o emulsificante é dado por:

$\frac{d\left([S] \cdot V_{R} \cdot \varphi\right)}{d t}=Q_{f} \cdot\left[S_{f}\right]$

onde, $\left[S_{f}\right]$ é a concentração de emulsificante na corrente de entrada do reator.

\subsubsection{Volumétrico}

O balanço volumétrico deve levar em conta a diferença de densidade entre 0 monômero e o polímero. Admite-se nesta equação, aditividade dos volumes dos componentes.

$$
\frac{d\left(V_{R}\right)}{d t}=\sum_{i}^{A, B}\left(\frac{1}{\rho_{p o l}}-\frac{1}{\rho_{i}}\right) \cdot\left(\Re_{p i}+\Re_{f i}\right) \cdot M M_{i}+Q_{f}
$$




\subsection{5 Água no Reator}

O balanço de água fica escrito na forma:

$\frac{d\left(V_{R} \cdot \varphi\right)}{d t}=Q_{f} \cdot \varphi_{f}$

onde, $\varphi_{f}$ é a fração volumétrica de água na corrente de entrada do reator.

\subsubsection{Agente de Transferência de Cadeia}

O balanço do agente de transferência é essencial para o modelo, pois este componente afeta diretamente as massas molares do polímero (BENYAHIA et al., 2010).

$\frac{d\left([A] \cdot V_{R}\right)}{d t}=[A] \cdot \sum_{i}^{A, B}\left(-k_{f A i} \cdot p_{i}\right) \cdot \int_{r_{n u c}}^{r_{\max }} \tilde{n}(r, t) \cdot d t+Q_{f} \cdot\left[A_{f}\right]$

onde $[A]$ é a concentração de agente de transferência de cadeia no reator, $k_{f A i}$ é a constante da taxa de transferência de cadeia para o monômero $i$ e $\left[A_{f}\right]$ é a concentração do agente de transferência de cadeia na corrente de entrada.

\subsection{Massas molares médias}

Na polimerização de radicais livres, as cadeias crescem em um período muito curto de tempo $(0,5$ - $10 \mathrm{~s})$ e o tamanho das macromoléculas depende das condições do meio em que elas crescem. Como o principal locus da polimerização é dentro das partículas de polímero, as massas molares médias são controlados pelo 
número de radicais por partícula (ASUA, 2007). As propriedades físicas e mecânicas dos polímeros dependem fortemente da distribuição de massas molares (BENYAHIA et al., 2010). As massas molares médias da polimerização em emulsão podem ser calculadas através do método dos momentos (ARAÚJO e GIUDICI, 2003; ARAÚJO e GIUDICI, 2004).

$\lambda_{k}=\sum_{n=1}^{\infty} n^{k} \cdot \dot{R}_{n}$

$\mu_{k}=\sum_{n=1}^{\infty} n^{k} \cdot D_{n}$

$\lambda_{o}=\int_{r_{\text {muc }}}^{r_{\max }} \tilde{n}(r, t) \cdot F(r, t) \cdot d r$

$\lambda_{1}=\frac{\lambda_{o} \cdot N_{A} \cdot V_{p} \cdot \sum_{i}^{A, B} k_{p i} \cdot\left[M_{i}\right]_{p}+\lambda_{o} \cdot N_{A} \cdot V_{p} \cdot \sum_{i}^{A, B} k_{f m i} \cdot\left[M_{i}\right]_{p}+k_{f p} \cdot \mu_{2} \cdot \lambda_{o}+\lambda_{o} \cdot N_{A} \cdot V_{p} \cdot k_{f A} \cdot[A]_{p}+k_{t} \cdot \lambda_{o}^{2}}{N_{A} \cdot V_{p} \cdot \sum_{i}^{A, B} k_{f m i} \cdot\left[M_{i}\right]_{p}+k_{f p} \cdot \mu_{1}+k_{t} \cdot \lambda_{o}+k_{f A} \cdot N_{A} \cdot V_{p}}$

$\lambda_{2}=\frac{\left(2 \cdot \lambda_{1}+\lambda_{o}\right) \cdot N_{A} \cdot V_{p} \cdot \sum_{i}^{A, B} k_{p i} \cdot\left[M_{i}\right]_{p}+\lambda_{o} \cdot N_{A} \cdot V_{p} \cdot \sum_{i}^{A, B} k_{f n i i} \cdot\left[M_{i}\right]_{p}+k_{f p} \cdot \mu_{3} \cdot \lambda_{o}+\lambda_{o} \cdot N_{A} \cdot V_{p} \cdot k_{f A} \cdot[A]_{p}+k_{t} \cdot \lambda_{o}^{2}}{N_{A} \cdot V_{p} \cdot \sum_{i}^{A, B} k_{f n n i} \cdot\left[M_{i}\right]_{p}+k_{f p} \cdot \mu_{1}+k_{t} \cdot \lambda_{o}+k_{f A} \cdot N_{A} \cdot V_{p}}$ 
As equações dos momentos dependem de parâmetros médios das constantes de taxa de reação que pode ser obtidos através das equações (4.73), (4.74), (4.75) e (4.76).

$$
\begin{aligned}
& k_{f m i}=\sum_{j}^{A, B} k_{f m i j} \cdot p_{j} \\
& k_{f p}=\sum_{i}^{A, B} k_{f p i} \cdot p_{i}
\end{aligned}
$$

$$
k_{t}=g \cdot \sum_{i}^{A, B} \sum_{j}^{A, B} k_{t i j} \cdot p_{i} \cdot p_{j}
$$

$$
k_{f A}=\sum_{i}^{A, B} k_{f A i} \cdot p_{i}
$$

$$
\frac{d\left(\mu_{o}\right)}{d t}=\lambda_{o} \cdot \sum_{i}^{A, B} k_{f m i} \cdot\left[M_{i}\right]_{p}+\frac{1}{2} \cdot \frac{k_{t c}}{N_{A} \cdot V_{p o l}} \cdot \lambda_{o}^{2}+\frac{k_{t d}}{N_{A} \cdot V_{p o l}} \cdot \lambda_{o}^{2}+k_{f A} \cdot[A]_{p} \cdot \lambda_{o}
$$

$$
\frac{d\left(\mu_{1}\right)}{d t}=\lambda_{o} \cdot \sum_{i}^{A, B} k_{p i} \cdot\left[M_{i}\right]_{p}+\lambda_{o} \cdot \sum_{i}^{A, B} k_{f m i} \cdot\left[M_{i}\right]_{p}+\frac{k_{t c}}{N_{A} \cdot V_{p o l}} \cdot \lambda_{o}^{2}+\frac{k_{t d}}{N_{A} \cdot V_{p o l}} \cdot \lambda_{o}^{2}+k_{f A} \cdot[A]_{p} \cdot \lambda_{o}
$$

$$
\frac{d\left(\mu_{2}\right)}{d t}=\left(2 \cdot \lambda_{1}+\lambda_{o}\right) \cdot \sum_{i}^{A, B} k_{p i} \cdot\left[M_{i}\right]_{p}+\lambda_{o} \cdot \sum_{i}^{A, B} k_{f i n i} \cdot\left[M_{i}\right]_{p}+\frac{k_{t c}}{N_{A} \cdot V_{p o l}} \cdot \lambda_{1}^{2}+\frac{k_{t d}}{N_{A} \cdot V_{p o l}} \cdot \lambda_{o}^{2}+k_{f A} \cdot[A]_{p} \cdot \lambda_{1}
$$


A relação entre $k_{t c}$ e $k_{t d}$ pode ser obtida através da equação (4.80) e (4.81), respeitando a premissa que o coeficiente da taxa de terminação é a somatória entre os termos de desproporcionamento e combinação (BENYAHIA et al., 2010).

$k_{t d}=\frac{\tau \cdot k_{t}}{1+\tau}$

$$
k_{t c}=\frac{k_{t}}{1+\tau}
$$

$$
M_{n}=\frac{\mu_{1}}{\mu_{o}} \cdot\left(\sum_{i}^{A, B} M M_{i} \cdot y_{i}\right)
$$

$$
M_{w}=\frac{\mu_{2}}{\mu_{1}} \cdot\left(\sum_{i}^{A, B} M M_{i} \cdot y_{i}\right)
$$

onde $y_{i}$ é a fração molar do monômero de tipo $i$ no copolímero formado. Pode-se ainda calcular o índice de polidispersidade IPD através da razão entre $M_{w}$ e $M_{n}$ conforme a equação (4.84).

$$
I P D=\frac{M_{w}}{M_{n}}
$$




\subsection{Conversão Global}

A conversão global pode ser calculada através da equação (4.85)

$$
X=\frac{\sum_{i}^{A, B} M M_{i} \cdot\left(\left[M_{i 0}\right]-\left[M_{i}\right]\right)}{\sum_{i}^{A, B} M M_{i} \cdot\left[M_{i 0}\right]}
$$

onde $X$ é a conversão global do processo. 


\section{MATERIAIS E MÉTODOS}

\subsection{Parâmetros utilizados nos modelos}

Modelos de polimerização em geral são dependentes de inúmeras constantes que determinam a qualidade dos resultados. A literatura apresenta diversas relações que nem sempre podem ser comparáveis, pois os procedimentos experimentais e os ajustes realizados raramente provêm os mesmos resultados. A Tabela 5.1 mostra cada um dos parâmetros, valor, unidade e a referência bibliográfica usados no modelo desenvolvido neste trabalho. Estes parâmetros não foram ajustados.

Tabela 5.1 - Parâmetros do modelo - A: Acrilato de Butila / B: Estireno

\begin{tabular}{|c|c|c|c|}
\hline Parâmetro & Valor & Unidade & Referência \\
\hline$k_{d, K P S}$ & $1,8 \cdot 10^{17} \cdot \exp (-17161 / T)$ & $s^{-1}$ & (RAWLINGS e RAY, 1988) \\
\hline$k_{d, N P S}$ & $2,6 \cdot 10^{17} \cdot \exp (-16719 / T)$ & $s^{-1}$ & (DUBÉ et al., 1997) \\
\hline$f$ & $\exp \left(-0,92 \cdot f_{b}\right)$ & - & (GINSBURGER et al., 2003) \\
\hline$k_{p A A}$ & $2,73 \cdot 10^{7} \cdot \exp (-3171 / T)$ & $\mathrm{m}^{3} \cdot \mathrm{kmol}^{-1} \cdot \mathrm{s}^{-1}$ & $\begin{array}{l}\text { (MCKENNA, GRAILLAT e } \\
\text { GUILLOT, 1995) }\end{array}$ \\
\hline$k_{p B B}$ & $4,57 \cdot 10^{7} \cdot \exp (-3921 / T)$ & $\mathrm{m}^{3} \cdot \mathrm{kmol}^{-1} \cdot \mathrm{s}^{-1}$ & (MANDERS et al., 1996) \\
\hline$r_{A B}$ & $\exp (1,3510-1034.1 / T)$ & - & $\begin{array}{l}\text { (WANG e HUTCHINSON, } \\
2010)\end{array}$ \\
\hline$r_{B A}$ & $\exp (0,05919-131.6 / T)$ & - & $\begin{array}{l}\text { (WANG e HUTCHINSON, } \\
2010)\end{array}$ \\
\hline$z_{A}$ & 1 & - & (ARAÚJO e GIUDICI, 2003) \\
\hline$z_{B}$ & 2 & - & (THICKETT e GILBERT, 2007) \\
\hline$k_{f m A A}$ & $5 \cdot 10^{-3} \cdot \exp (7,45-2405 / T)$ & $\mathrm{m}^{3} \cdot \mathrm{kmol}^{-1} \cdot \mathrm{s}^{-1}$ & (GINSBURGER et al., 2003) \\
\hline$k_{f m B B}$ & $8,636 \cdot 10^{-3} \cdot \exp (20,8-6723 / T)$ & $\mathrm{m}^{3} \cdot \mathrm{kmol}^{-1} \cdot \mathrm{s}^{-1}$ & (MEDOWS et al., 2003) \\
\hline$k_{f p A}$ & $2,48 \cdot 10^{3} \cdot \exp (-27700 / T)$ & $\mathrm{m}^{3} \cdot \mathrm{kmol}^{-1} \cdot \mathrm{s}^{-1}$ & (HAMZEHLOU et al., 2014) \\
\hline$k_{f p B}$ & $1,9 \cdot 10^{-4} \cdot k_{p B B}$ & $\mathrm{~m}^{3} \cdot \mathrm{kmol}^{-1} \cdot \mathrm{s}^{-1}$ & (HAMZEHLOU et al., 2014) \\
\hline$k_{f a A}$ & $0,4 \cdot 7,166 \cdot 10^{11} \cdot \exp (-7577 / T)$ & $\mathrm{m}^{3} \cdot \mathrm{kmol}^{-1} \cdot \mathrm{s}^{-1}$ & (BENYAHIA et al., 2010) \\
\hline
\end{tabular}




\begin{tabular}{|c|c|c|c|}
\hline$k_{f a B}$ & $0,4 \cdot 1,286 \cdot 10^{8} \cdot \exp (-4089 / T)$ & $\mathrm{m}^{3} \cdot \mathrm{kmol}^{-1} \cdot \mathrm{s}^{-1}$ & (BENYAHIA et al., 2010) \\
\hline$k_{t A A}$ & $7,5 \cdot 10^{14} \cdot \exp (-9216 / T)$ & $\mathrm{m}^{3} \cdot \mathrm{kmol}^{-1} \cdot \mathrm{s}^{-1}$ & $\begin{array}{ll}\text { (CASELLA, } & \text { ARAUJO } \\
\text { GIUDICI, 2003) } & \end{array}$ \\
\hline$k_{t B B}$ & $1,341 \cdot 10^{10} \cdot \exp (-1725 / T)$ & $\mathrm{m}^{3} \cdot \mathrm{kmol}^{-1} \cdot \mathrm{s}^{-1}$ & (TAYLOR et al., 2010) \\
\hline$j_{\text {crit } A}$ & 8 & - & (ARAÚJO e GIUDICI, 2003) \\
\hline$j_{\text {crit } B}$ & 5 & - & (THICKETT e GILBERT, 2007) \\
\hline$D_{a q, A}$ & $1,1 \cdot 10^{-9}$ & $m^{2} \cdot s^{-1}$ & (ARAÚJO e GIUDICI, 2003) \\
\hline$D_{a q, B}$ & $1,5 \cdot 10^{-9}$ & $m^{2} \cdot s^{-1}$ & (ZEAITER et al., 2002) \\
\hline$D_{p, A}$ & $1,1 \cdot 10^{-10}$ & $m^{2} \cdot s^{-1}$ & $\begin{array}{ll}\text { (CASELLA, } & \text { ARAUJO } \\
\text { GIUDICI, 2003) } & \end{array}$ \\
\hline$D_{p, B}$ & $1,5 \cdot 10^{-10}$ & $m^{2} \cdot s^{-1}$ & $\begin{array}{l}\text { (CASELLA, } \\
\text { GIUDICI, 2003) }\end{array}$ \\
\hline$K_{d}^{A}$ & 471 & - & (ARBINA et al., 1997) \\
\hline$K_{p}^{A}$ & 724 & - & (ARBINA et al., 1997) \\
\hline$K_{d}^{B}$ & 2714 & - & (ARBINA et al., 1997) \\
\hline$K_{p}^{B}$ & 1629 & - & (ARBINA et al., 1997) \\
\hline$r_{m i c}$ & $2,5 \cdot 10^{-9}$ & $m$ & (GILBERT, 1995) \\
\hline$\tau$ & $2 / 3$ & - & (BENYAHIA et al., 2010) \\
\hline$\theta$ & 1,03 & - & (BENYAHIA et al., 2010) \\
\hline$K_{p A}$ & 0,55 & - & (BENYAHIA et al., 2010) \\
\hline$k_{f A, a q} \cdot A_{d}$ & $5 / 6$ & $m^{3} \cdot s^{-1}$ & (SALAZAR et al., 1998) \\
\hline$B_{A}$ & 2,23 & - & (PLESSIS et al., 2001) \\
\hline$C_{A}$ & 0 & - & (PLESSIS et al., 2001) \\
\hline$D_{A}$ & 0 & - & (PLESSIS et al., 2001) \\
\hline$m_{A}$ & 1 & - & (PLESSIS et al., 2001) \\
\hline$B_{B}$ & $2,57-0,00505 \cdot T$ & - & (FRIIS e HAMIELEC, 1976) \\
\hline$C_{B}$ & $9,56-0,0176 \cdot T$ & - & (FRIIS e HAMIELEC, 1976) \\
\hline$D_{B}$ & $-3,03-0,00785 \cdot T$ & - & (FRIIS e HAMIELEC, 1976) \\
\hline$m_{B}$ & 2 & - & (FRIIS e HAMIELEC, 1976) \\
\hline
\end{tabular}




\subsection{Solução numérica dos modelos}

A solução numérica das equações diferenciais e do balanço populacional foi realizada utilizando como plataforma o MATLAB 7.1 R2010a. O problema descrito foi resolvido através da solução numérica de um conjunto de equações algébricodiferenciais, onde a solução da partição de monômeros, assim como a solução da concentração de radicais na fase aquosa foi resolvida através da solução de sistema de equações não lineares. Assim, um processo iterativo foi implementado para a solução do conjunto de equações não lineares e o mesmo é acionado a cada passo de integração.

A solução das equações de partição dos monômeros entre as fases, equações (4.37) a (4.48), foram obtidas através de um algoritmo iterativo descrito na literatura (OMl et al., 1985).

O método usado para solução do conjunto de equações diferenciais é baseado em fórmulas de diferenciação numérica (NDF) de ordem variada (ODE15S). Vale ressaltar que o sistema de equações é stiff devido às diferentes ordens de grandeza encontradas das variáveis do problema, e principalmente devido à presença de balanços de espécies de diferentes naturezas (radicais livres e espécies não radicalares). As equações diferenciais podem ser divididas em três grandes grupos: balanço de massa, balanço de momentos e balanços populacionais. Neste último conjunto, cada classe é uma equação diferencial, considerando o número de partículas e número médio de radicais por partícula.

Outras dificuldades encontradas na solução de modelos de polimerização em emulsão são relativas à intensidade numérica na solução do sistema de equações diferenciais não-lineares acopladas às equações algébricas e a falta de informação de parâmetros (GAO e PENLIDIS, 2002).

Alguns autores recomendam que a solução numérica das equações diferenciais e das equações algébricas não-lineares sejam feitas em conjunto através do método de DDASSL (Differential Algebric System Solver), apresentando bons resultados (IMMANUEL et al., 2002; IMMANUEL e DOYLE III, 2003). 
Um modelo auxiliar simplificado foi construído de modo que a distribuição de tamanho de partículas fosse considerada monodispersa. Este modelo foi construído para que os ajustes de parâmetros pudessem ser feitos com maior agilidade, pois o conjunto de equações diferenciais a ser resolvido é reduzido consideravelmente e consequentemente se reduz o tempo computacional da solução numérica.

Diferentes abordagens encontradas em literatura (GINSBURGER et al., 2003; BENYAHIA et al., 2010) utilizam técnicas de otimização para ajustar um conjunto considerável de parâmetros (inclusive constantes cinéticas de propagação), o que leva a modelos melhor ajustados aos dados experimentais, porém com menor capacidade preditiva para condições experimentais distintas daquelas para as quais o modelo foi ajustado. A abordagem escolhida para a esta modelagem teve como objetivo construir um modelo que primasse pela generalidade, ou seja, que o mesmo pudesse ser usado em diversos casos, portanto somente são ajustados dois parâmetros.

Vale ressaltar que alguns autores abordam o problema através de métodos estocásticos, pois consideram que as soluções pelos métodos tradicionais não levam em conta a característica estocástica do crescimento das partículas na polimerização em emulsão (HOSSEINI, BOUASWAIG e SEBASTIAN, 2012).

A Figura 5.1 mostra o fluxograma de todas as etapas consideradas na modelagem matemática para a simulação do processo de copolimerização em emulsão isotérmico em reator tipo batelada ou semi-batelada. Os parâmetros considerados no modelo são de acrilato de butila e estireno. Portanto, para a simulação de outro conjunto de monômeros, os parâmetros devem ser ajustados e caso necessário, as equações dos fenômenos físico-químicos. Os parâmetros $f_{k e}$ e $f_{\text {kemic }}$ devem ser ajustados para um conjunto de experimento para que possam ser utilizados em simulações com condições operacionais diferentes.

O Apêndice C apresenta o programa completo em linguagem MATLAB 7.1 R2010a que pode ser utilizado e evoluído para futuras pesquisas em temas afins, ou mesmo adaptado para aplicações em processos industriais. As equações utilizadas são os intervalos de (4.2) a (4.8) e (4.13) a (4.83). Ainda, foram usadas as equações (5.1), (5.2) e (5.3) no intuito de melhorar a robustez da solução. 


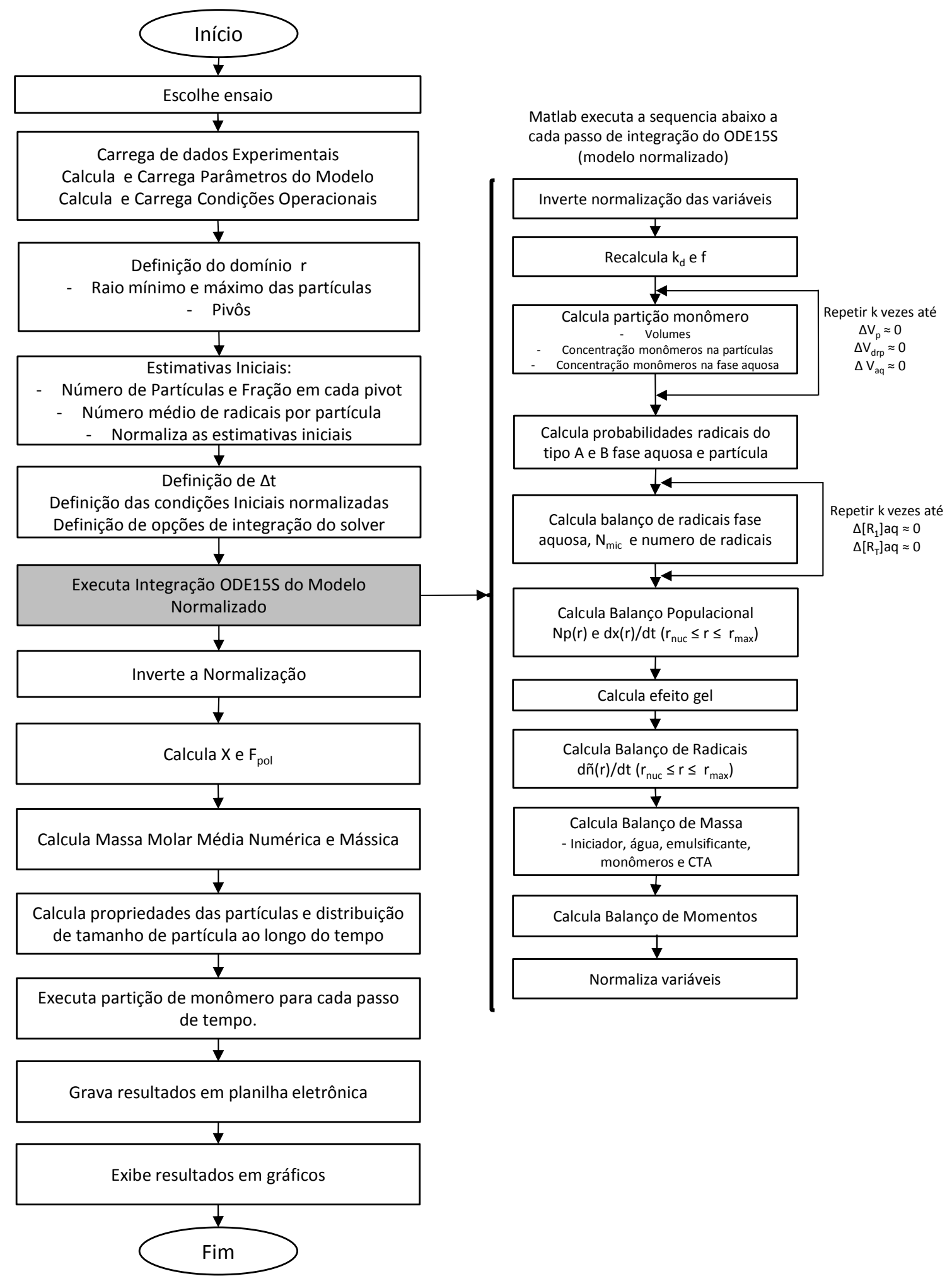

Figura 5.1 - Fluxograma do modelo implementado 


\subsubsection{Solução Balanço Populacional}

Diferentes métodos vêm sendo empregados para a solução das equações de balanço populacional. Existem dois tipos de modelo para tal, os modelos de nível 1 (level-one models), que são baseados na aproximação de uma distribuição monodispersa, ou seja, as partículas têm o mesmo volume médio. Em contrapartida os modelos de nível 2 (level-two models) consideram a DTP através de balanços populacionais. Os métodos mais usados para a solução de equações de balanço populacional são (VALE e MCKENNA, 2005):

- Colocação ortogonal em Elementos Finitos: as equações de balanço populacional são semi-discretizadas e transformadas de um sistema de equações algébricas, onde as incógnitas são os valores de densidade populacional nos pontos de colocação ortogonal. Estes modelos em geral não consideram efeitos de coagulação, pois apresentam problemas de difusão numérica.

- Diferenças finitas: método de simples implementação para resolução de problemas equações diferenciais parciais que pode ser aplicado para a resolução de balanços populacionais com algumas vantagens, entretanto pode apresentar limitações quando aplicado a problemas hiperbólicos.

- Volumes finitos (Pivots Fixos): relacionado ao método das diferenças finitas, é o método que apresenta vantagens como a mitigação da difusão numérica devido à natureza hiperbólica das equações de balanço populacional, garante que o número de partículas é conservado; apresenta facilidade para alocação da fonte de nucleação e a formulação é compatível com os métodos de discretização desenvolvidos para a coagulação. 
O método dos pivots fixos foi utilizado para discretizar o domínio de tamanho de partículas em classes igualmente espaçadas. Cada classe representa um diâmetro de partícula no balanço populacional. O balanço populacional para o número de radicais por partícula e para o número de partículas formam um sistema de equações diferenciais, onde cada classe representa uma equação. $O$ termo de nucleação das partículas é aplicado somente na primeira classe, onde o raio da partícula é igual ao raio da micela.

A normalização das variáveis do sistema de equações foi realizada para ajudar a convergência da solução numérica. No intuito de construir um modelo genérico que pode ser executado tanto para o sistema em semi-batelada quanto em batelada, adequou-se o balanço populacional separando o número total de partículas e a fração de partículas em cada pivot. As equações (5.1), (5.2) e (5.3) foram implementadas no modelo. A condição de não-negativos foi ativada no solver para todos os resultados, garantindo estabilidade ao modelo.

$$
N_{p}=N_{p i} \cdot x_{i}
$$

$$
\frac{d N_{p}}{d t}=\Re_{n u c}
$$

$$
\frac{d x_{i}}{d t}=\frac{\left[\frac{d N_{p i}}{d t}-x_{i} \cdot \Re_{\text {uuc }}\right]}{N_{p}}
$$

onde $x_{i}$ é fração de partícula presentes em cada pivot $i$ e $N_{p i}$ é o número de partículas em cada pivot $i$. O termo $d N_{p i} / d t$ pode ser calculado através do balanço populacional. 


\subsection{Experimentos e dados da Literatura}

\subsubsection{Experimentos (ARAÚJO, 1997)}

Um planejamento de experimentos foi realizado no intuito de investigar experimentalmente diferentes condições operacionais para diferentes combinações e monômeros em sistemas isotérmicos em batelada. Os monômeros utilizados foram estireno, metacrilato de metila, acrilato de butila, acetato de vinila, butadieno, ácido acrílico e etilenos. O iniciador utilizado foi o persulfato de amônia e o emuslficante utilizado foi dodecil sulfato de sódio. Os experimentos cobrem uma grande variação de concentração de iniciador, concentração de emulsificante, razão entre monômero e temperatura.

\subsubsection{Materiais e Condições Operacionais}

A Tabela 5.2 mostra as condições operacionais experimentais realizadas para os experimentos em batelada. $O$ tempo de processo para todos os experimentos foi de 120 minutos.

Tabela 5.2 - Condições dos experimentos (ARAÚJO, 1997)

\begin{tabular}{c|c|c|c|c|c}
\hline Experimento & $\begin{array}{c}\mathrm{T} \\
\left({ }^{\circ} \mathrm{C}\right)\end{array}$ & $\begin{array}{c}\mathrm{B} / \mathrm{A} \\
(\% \mathrm{molar})\end{array}$ & $\begin{array}{c}\text { Iniciador } \\
\left(\mathrm{kmol} / \mathrm{m}^{3}\right)\end{array}$ & $\begin{array}{c}\text { Emulsificante } \\
\left(\mathrm{kmol} / \mathrm{m}^{3}\right)\end{array}$ & $\begin{array}{c}\text { Monômero/Água } \\
(\mathrm{kg} / \mathrm{kg} \text { água })\end{array}$ \\
\hline BSBA-1 & 60 & $70 / 30$ & $410^{-3}$ & $2,810^{-2}$ & 0,34 \\
BSBA-5 & 60 & $30 / 70$ & $210^{-3}$ & $2,810^{-2}$ & 0,34 \\
BSBA-6 & 60 & $70 / 30$ & $210^{-3}$ & $2,810^{-2}$ & 0,34 \\
BSBA-7 & 60 & $70 / 30$ & $210^{-3}$ & $1,410^{-2}$ & 0,55 \\
BSBA-8 & 60 & $30 / 70$ & $410^{-3}$ & $1,410^{-2}$ & 0,34 \\
BSBA-9 & 60 & $30 / 70$ & $210^{-3}$ & $2,810^{-2}$ & 0,55 \\
BSBA-12 & 60 & $70 / 30$ & $210^{-3}$ & $1,410^{-2}$ & 0,34 \\
BSBA-13 & 60 & $30 / 70$ & $210^{-3}$ & $1,410^{-2}$ & 0,34 \\
BSBA-2 & 70 & $70 / 30$ & $210^{-3}$ & $1,410^{-2}$ & 0,55
\end{tabular}




\begin{tabular}{c|c|c|c|c|c} 
BSBA-3 & 70 & $30 / 70$ & 2 & 1,4 & 0,34 \\
BSBA-4 & 70 & $70 / 30$ & 2 & 1,4 & 0,34 \\
BSBA-10 & 70 & $30 / 70$ & 4 & 2,8 & 0,34 \\
BSBA-11 & 70 & $70 / 30$ & 4 & 1,4 & 0,34 \\
\hline
\end{tabular}

A: Acrilato de Butila / B: Estireno

\subsubsection{Equipamento}

Os experimentos foram conduzidos em um reator de 2,4 litros encamisado, equipado com um agitador magnético e uma turbina de quatro pás, operando a 250 RPM. O controle de temperatura foi realizado por meio de um banho de água com uma bomba de circulação e um controlador (CN76000 Omega). A temperatura do banho orbitava em torno de $0,5^{\circ} \mathrm{C}$ em relação a temperatura desejada.

\subsubsection{Métodos Analíticos}

A conversão foi calculada através de técnicas gravimétricas e o tamanho de partícula por espalhamento de luz dinâmica. Deste modo, amostras foram retiradas periodicamente do reator e a polimerização era cessada adicionando-se a solução de hidroquinina (1\%), previamente colocada em cápsulas de alumínio. A massa do látex úmido foi medida e as cápsulas foram conduzidas para a secagem até a que a massa seja constante.

\subsubsection{Experimentos (MARINANGELO, 2010)}

Os experimentos realizados pelo grupo de polimerização do Departamento de Engenharia Química da Escola Politécnica da USP e serviram como validação do modelo. 


\subsubsection{Materiais e Condições Operacionais}

Acrilato de butila e estireno fornecidos pela Rhodia e usados sem purificação prévia, ambos com 50 ppm de Inibidor. Ultrawet 230 da Oxiteno e Disponil 25S da Cognis foram usados como emulsificantes não-iônico e iônico respectivamente. Persulfato de sódio foi utilizado como iniciador e N-dodecilmercaptana como agente de transferência de cadeia. Água deionizada foi usada em todos os experimentos e processos analíticos. A Tabela 5.3 mostra as condições operacionais e a formulação de cada experimento.

Tabela 5.3 - Condições dos experimentos (MARINANGELO, 2010)

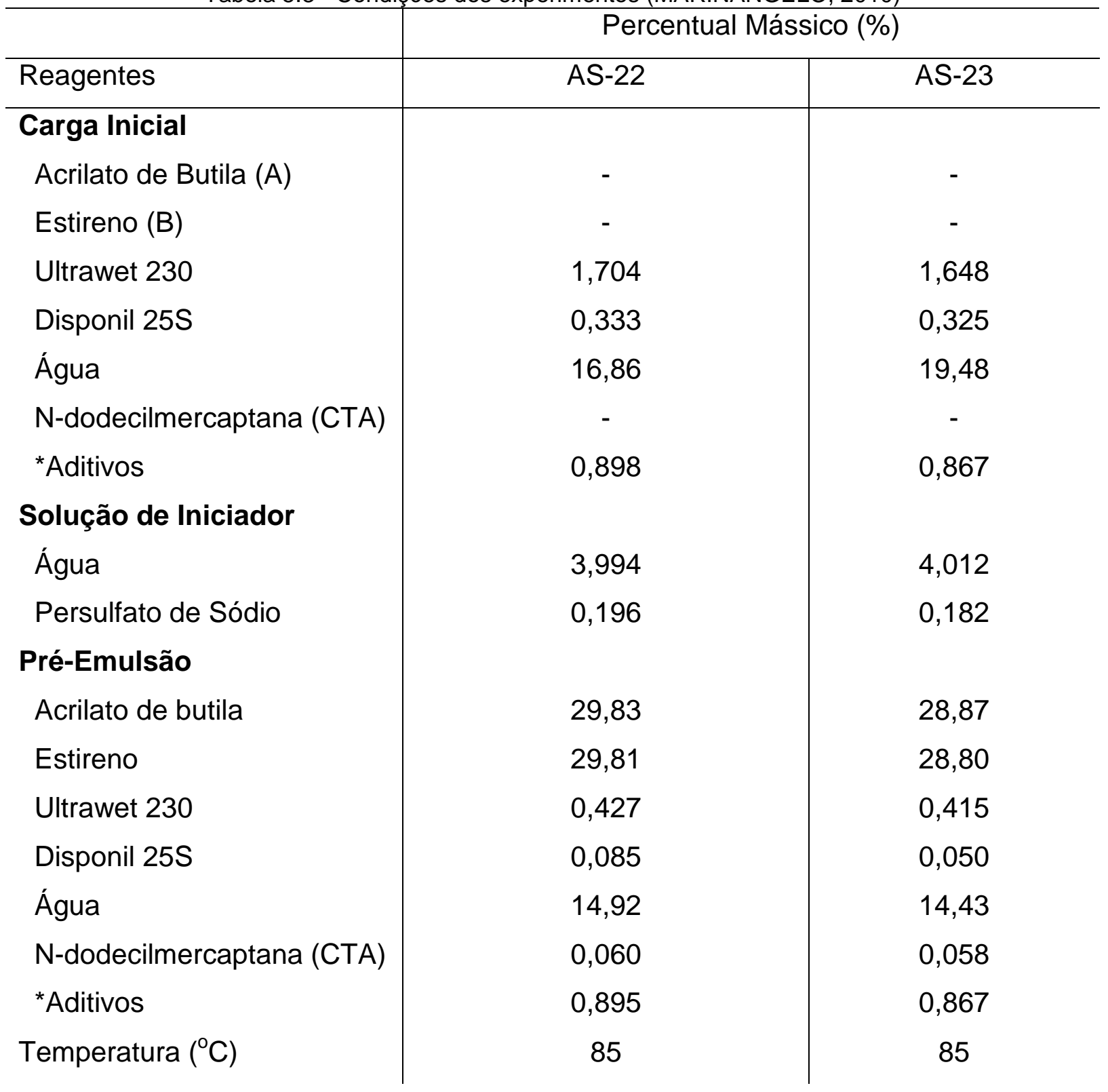




\begin{tabular}{l|c|c} 
Tempo Total de alimentação & 210 & 210 \\
$\begin{array}{l}\text { Solução Iniciador (min) } \\
\text { Tempo total de Alimentação }\end{array}$ & 180 & 180 \\
de pré-emulsão (min) & & \\
\hline
\end{tabular}

*Aditivos: Acido Acrílico; $\mathrm{NaHCO}_{3}$

\subsubsection{Equipamento}

Um reator de vidro encamisado com um agitador de quatro pás foi usado para os experimentos. A temperatura do reator foi controlada por dois banhos com solução de etileno glicol aquecida e gelada. As soluções eram misturadas na camisa do reator. A alimentação da pré-emulsão era controlada por uma bomba de diafragma e checada com uma balança eletrostática. A alimentação da solução de iniciação era feita através de uma bomba peristáltica a partir de uma bureta. Um condensador com carvão ativado foi usado para reduzir as perdas de monômero (MARINANGELO, HIROTA e GIUDICI, 2011).

\subsubsection{Métodos Analíticos}

Durante os ensaios de polimerização, amostras foram retiradas periodicamente do reator e a polimerização era cessada adicionando-se $0,5 \mathrm{~g}$ de solução de hidroquinina (1\%). A conversão de monômeros foi analisada através de gravimetria. Amostras foram coletadas periodicamente e acondicionadas em capsulas de alumínio contendo de $0,3 \mathrm{~g}$ a $0,5 \mathrm{~g}$ de solução de hidroquinona (1\%). A massa do látex úmido foi medida e as capsulas foram conduzidas para a estufa a $100^{\circ} \mathrm{C}$ por um período de 24 horas, ou até que a massa seja constante. A fração de sólidos na amostra pode ser determinada pela razão entre a massa do látex após secagem e a massa do látex previamente à secagem, conforme a equação (5.4).

O diâmetro médio das partículas foi determinado por espalhamento de luz dinâmica e espectroscopia de correlação de fótons (DLS/PCS) em um equipamento Coulter N4Plus. A concentração de monômeros no reator foi determinada por cromatografia gasosa em um equipamento Shimadzu HSS-4A. A distribuição de 
tamanho de partícula foi pautada em análises de imagens por meio de um microscópio de transmissão eletrônica.

\subsection{Análise Gravimétrica}

A análise gravimétrica é um método simples e baixo custo, que não requer muitos equipamentos, apresentando resultados precisos, pois a medida é feita através de relações estequiométricas com auxilio de uma balança calibrada. A principal desvantagem do método de gravimétrico está na necessidade de grande quantidade de amostras e consequentemente acumulação de erros. Entre os métodos gravimétricos, pode-se destacar a precipitação e volatilização. A fração de sólidos no reator pode ser calculada pela equação (5.4).

$$
F_{S}=\frac{M_{3}-M_{1}}{M_{2}-M_{4}}
$$

onde, $F_{S}$ é a fração de sólidos na amostra, $M_{1}$ é a massa da placa vazia, $M_{2}$ é a massa da placa com amostra úmida, $M_{3}$ é a massa da placa com amostra seca, e $M_{4}$ é a massa da placa com solução de hidroquinona.

A fração de polímeros na amostra pode ser obtida subtraindo a fração de aditivos da fração de sólidos não polimerizáveis, contida nos aditivos.

$$
F_{P}=F_{S}-F_{T}
$$

$$
F_{T}=\frac{M_{5}}{M_{T}}
$$

onde, $F_{P}$ é a fração de polímeros na amostra, $F_{T}$ é a fração total de sólidos não polimerizáveis, $M_{5}$ é a massa de sólidos não polimerizáveis adicionados e $M_{T}$ é a massa total da amostra. 
A conversão global de monômeros pode ser calculada através da razão entre a fração de polímero na amostra e a fração de monômero total na receita, conforme mostrado pela equação (5.7).

$$
X=\frac{F_{p}}{F_{M T}}
$$

onde $X$ é a conversão global e $F_{M T}$ é a fração de monômero total na receita.

\subsection{Diâmetro das Partículas.}

A difração dinâmica de luz é utilizada na área de polímeros para determinar o diâmetro médio das partículas poliméricas. Através da medida da velocidade de difusão de partículas pelo movimento browniano, ou seja, à medida que as partículas se movimentam durante a passagem do feixe de luz, o tipo de interferência e a intensidade da luz são detectados a cada instante fornecendo assim a taxa de difusão das partículas na amostra, a partir da qual o diâmetro médio é obtido (HIROTA, 2009).

\subsection{Número de Partículas no Reator.}

A equação (5.8) permite calcular o diâmetro das partículas e se pode calcular a concentração média de partículas no látex.

$$
\left[N_{p}\right]=\frac{F_{p}}{\frac{\rho_{p o l}}{\rho_{l}}\left(\frac{\pi}{6} d_{p}^{3}\right)}
$$

onde, $\left\lfloor N_{p}\right\rfloor$ é o número de partículas por litro no reator, $\rho_{l}$ é a densidade do látex e $d_{p}$ é o diâmetro da partícula. 


\subsection{Taxa de Polimerização}

A taxa de polimerização entre dois instantes, $t$ e $t+\Delta t$ pode ser calculada através da equação (5.9) (MARINANGELO, 2010).

$$
R_{p}=\frac{\left\lfloor F_{p} \cdot M_{\text {total }}\right\rfloor_{t+\Delta t}-\left\lfloor F_{p} \cdot M_{\text {total }}\right\rfloor_{t}}{\Delta t}
$$

onde $R_{p}$ é a taxa de polimerização e $M_{\text {total }}$ é a massa adicionada ao reator até o instante $t$ indicado.

\subsection{Número médio de radicais por partícula}

O número médio de radicais por partículas foi obtido a partir dos coeficientes de partição entre os monômeros e dos volumes de monômero, água e partículas no reator. Os volumes foram obtidos através do balanço de massa. O mesmos parâmetros utilizados na modelagem, foram aplicados na obtenção dos dados experimentais.

$$
[M]_{p}=\left(\frac{V_{p}^{m}}{V_{p}^{m}+V_{p}^{p}}\right) \cdot \frac{\rho_{\text {mon }}}{M M_{m o n}}
$$

onde, $V_{p}^{m}$ é o volume de monômero na fase polimérica, $V_{p}^{p}$ é volume de polímeros na fase polimérica, $\rho_{m o n}$ é a densidade média dos monômeros e $M M_{m o n}$ é a massa molar média dos monômeros. O número médio de radicais por partícula pode ser calculado através da taxa de polimerização e concentração e monômeros no reator. O valor da constante de propagação $k_{p}$ médio pode ser calculado através de relações apresentadas na literatura (KONG, PICHOT e GUILLOT, 1988). 


$$
R_{p}=k_{p} \cdot[M]_{p} \cdot \frac{N_{p}}{N_{A}} \cdot \tilde{n} \cdot M M_{\text {mon }}
$$

Vale ressaltar que estas relações empíricas podem apresentar desvio para os cálculos dos resultados do sistema.

\subsubsection{Experimentos (BENYAHIA et al., 2010)}

Os experimentos obtidos da literatura foram utilizados para validar o modelo construído. Neste tópico o foco principal está relacionado à validação dos resultados de simulação para a massa molar média numérica e mássica.

\subsubsection{Materiais e Condições Operacionais}

Acrilato de butila e estireno previamente estabilizados com 15 ppm de 4terbutilcatecol adquirido da Acros Organics. Persulfato de Amônio fornecido pela Sigma-Aldrich foi utilizado como iniciador para estes experimentos. O surfactante escolhido foi REWOPOL SBFA 50 adquirido da Goldschimidt. O Agente de transferência de cadeia escolhido foi o n-dodecilmercaptana, também da Acros Organics.

Tabela 5.4 - Condições dos experimentos (BENYAHIA et al., 2010)

\begin{tabular}{l|c|c|c|c}
\hline & \multicolumn{4}{|c}{ Massa (g) } \\
\hline Experimentos & $\mathrm{R} 1$ & $\mathrm{R} 2$ & $\mathrm{R} 3$ & $\mathrm{R} 4$ \\
\hline Carga Inicial & & & & \\
Acrilato de butila & 60 & 60 & 60 & 60 \\
Estireno & 60 & 60 & 60 & 60 \\
Rewopol SBFA & 15 & 15 & 15 & 15 \\
50 & 570 & 570 & 570 & 570 \\
Água & 0,3 & 0,6 & 0,3 & 0,6 \\
N- & & & &
\end{tabular}




\begin{tabular}{|c|c|c|c|c|}
\hline $\begin{array}{l}\text { dodecilmercaptan } \\
\text { a (CTA) }\end{array}$ & & & & \\
\hline $\begin{array}{l}\text { Persulfato de } \\
\text { Amônio }\end{array}$ & 1 & 1 & 1 & 1 \\
\hline Temperatura $\left({ }^{\circ} \mathrm{C}\right)$ & 60 & 60 & 70 & 70 \\
\hline $\begin{array}{l}\text { Tempo total da } \\
\text { batelada (min) }\end{array}$ & 100 & 100 & 100 & 100 \\
\hline
\end{tabular}

\subsubsection{Equipamento}

Um reator de vidro de 1 litro equipado com um agitador de aço inox, um condensador de refluxo, um criostato, uma entrada de nitrogênio e um equipamento para retirada de amostras. O misturador é composto de um agitador fixo no fundo. A rotação é mantida a $200 \mathrm{rpm}$ e a temperatura constante durante todo o experimento. Amostras eram tiradas do reator em intervalos de tempos apropriados e a polimerização era cessada com hidroquinina a baixa temperatura (BENYAHIA et al., 2010).

\subsubsection{Métodos Analíticos}

As amostras recuperadas dos experimentos foram caracterizadas em termos de conversão global por gravimetria usando um analisador halógeno de umidade Mettler Toledo HG53. Aproximadamente $1 \mathrm{~g}$ látex foi colocado em uma placa de alumínio que foi introduzida no analisador e aquecida a $175^{\circ} \mathrm{C}$ até a evaporação completa da água e monômeros residuais. A massa seca final foi medida automaticamente. Foram realizadas correções da quantidade de iniciador e surfactantes residuais e a conversão final foi então calculada.

No intuito de controlar o consumo de cada monômero durante a polimerização, uma determinação quantitativa mais precisa destes monômeros foi necessária. A técnica de cromatografia a gás foi escolhida utilizando um VARIAN CG3900 equipado com uma coluna capilar e com uma pré-coluna de aço inox recheada de fibras de vidro. O diâmetro das partículas foi determinado usando um 
Malvern 4700 quasi-elástico de varredura de luz, após a diluição com água deionizada, o diâmetro médio das partículas foi medido (BENYAHIA et al., 2010).

A massa molar média numérica e mássica foi determinada por cromatografia de exclusão, usando um refratômetro diferencial como detector. Os dados coletados no detector foram analisados, utilizando o software Astra da Wyatt Tecnologia, o que permite a determinação a distribuição de massa molar e as massas molares médias numéricas e mássicas. 


\section{RESULTADOS E DISCUSSÃO}

Neste capítulo, são apresentados os resultados de validação do modelo desenvolvido, comparando os mesmos com dados experimentais disponíveis na literatura, de diferentes fontes, obtidos sob distintas condições experimentais. Somente serão apresentados neste capítulo os resultados do modelo passíveis de serem comparados com experimentos. Os parâmetros foram ajustados por tentativa e erro.

\subsection{Experimentos (ARAÚJO, 1997)}

Os parâmetros $f_{k e}$ e $f_{\text {kemic }}$, apresentados na Tabela 6.1, foram ajustados por tentativa e erro para que os dados do experimento BSBA-1 tivessem maior aderência com os resultados do modelo. Os parâmetros ajustados foram utilizados para simular os outros experimentos. As propriedades do emulsionante também são apresentadas devido à escolha de cada autor.

Tabela 6.1 - Parâmetros estimados na modelagem (ARAÚJO, 1997)

\begin{tabular}{|c|c|c|c|}
\hline$f_{k e}$ & $f_{\text {kemic }}$ & ${ }^{*} \mathrm{CMC}\left(\mathrm{kmol} / \mathrm{m}^{3}\right)$ & $\begin{array}{l}{ }^{*} a_{e}=a_{s} \cdot N_{A}^{-1} \\
\left(\mathrm{~m}^{2} / \text { molécula }\right)\end{array}$ \\
\hline $0,5 \cdot 10^{-4}$ & $1,5 \cdot 10^{-5}$ & $2,43 \cdot 10^{-3}$ & $5 \cdot 10^{-19}$ \\
\hline
\end{tabular}

*parâmetros disponíveis em literatura

Os resultados de conversão e diâmetros médios das partículas obtidos experimentalmente foram comparados aos resultados do modelo. As condições de operacionais e a receita foram apresentadas na Tabela 5.2. $O$ valor de $f_{\text {kemic }}$ apresentou a mesma ordem de grandeza quando comparado aos valores apresentados na literatura para o mesmo conjunto de experimentos (CASELLA, ARAUJO e GIUDICI, 2003). Entretanto, o parâmetro $f_{k e}$ obtido foi muito menor. 


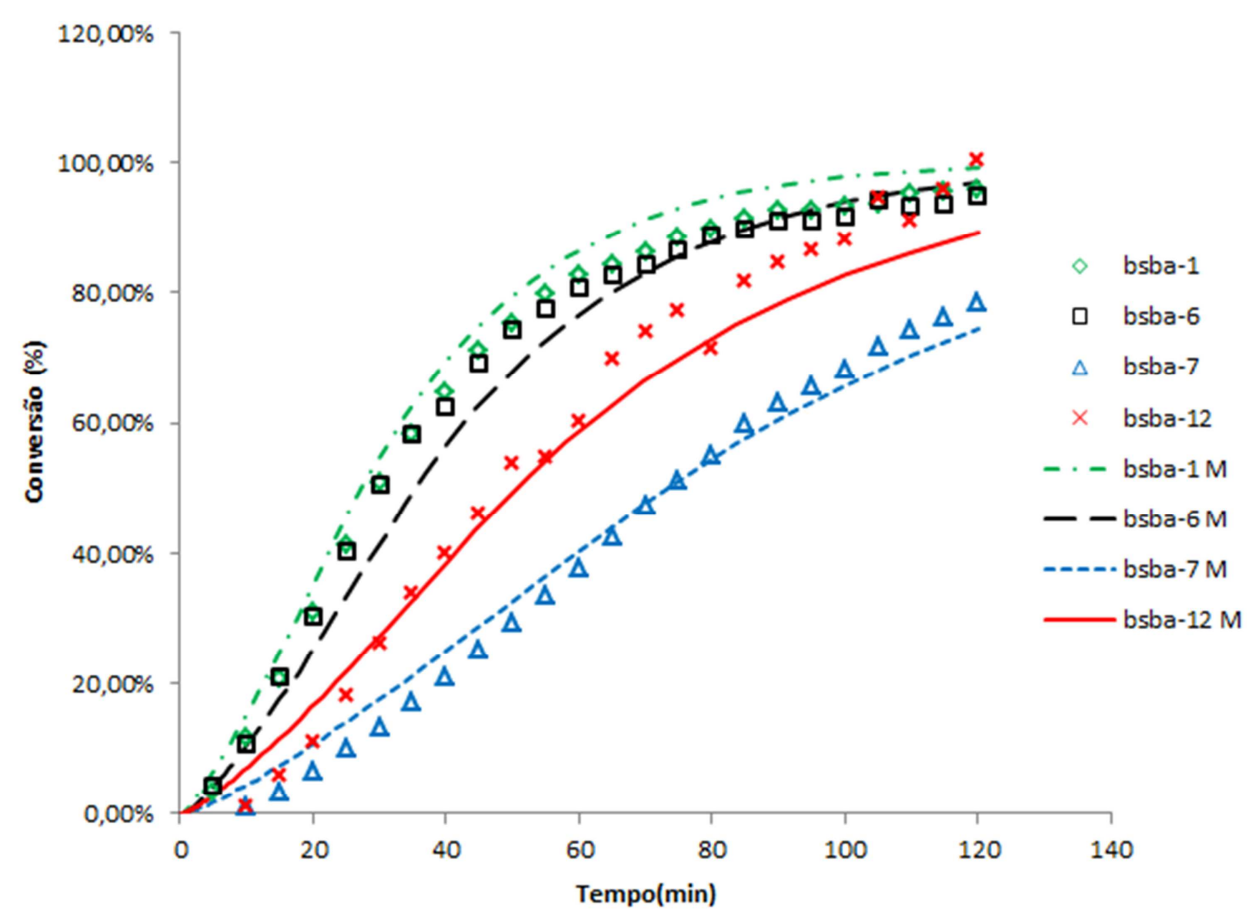

Figura 6.1 - Conversão Experimentos BSBA-1, BSBA-6, BSBA-7 e BSBA-12

Os experimentos apresentados na Figura 6.1 são todos rodados a temperatura de $60^{\circ} \mathrm{C}$ e apresentam uma razão de monômero 30/70 para acrilato de butila e estireno. Mostra-se que o modelo tem uma boa aderência com os valores experimentais mesmo com a mudança das condições operacionais. Neste caso, o perfil da curva variou com as diferenças na quantidade de emulsificante, na concentração de iniciador e na razão entre monômero e água.

A concentração de emulsificante determina o número de partículas no reator e consequentemente a taxa de propagação. Considerando que os ensaios bsba-1 e bsba-6 apresentam as maiores concentrações de emulsificante, nota-se que as taxas de propagação foram maiores, conforme apresentado nas curvas de conversão da Figura 6.1.

O diâmetro médio das partículas em relação à conversão pode ser observado na Figura 6.2. Observa-se que o modelo não tem o mesmo patamar de erro que os resultados de conversão, sendo pior, entretanto consegue capturar qualitativamente a relação entre o diâmetro médio e a conversão dos experimentos.

Observa-se que a concentração de emulsificante nos ensaios BSBA-1 e BSBA-6 são o dobro do que aquelas apresentadas pelos experimentos BSBA-7 e BSBA-12. Portanto, considerando a equação (4.53), espera-se um aumento no 
número de micelas e de partículas, proporcionando redução no diâmetro médio das mesmas. O comportamento é descrito na Figura 6.2, tanto para os dados experimentais quanto para os resultados do modelo.

Vale ressaltar que medidas abaixo de $20 \%$ de conversão, onde há baixa concentração de partículas, são difíceis de obter devido às limitações da técnica de espalhamento de luz, onde partículas maiores têm maior peso no cálculo da média. Em baixas conversões, a maioria das partículas é pequena, mas poucas partículas grandes interferem na medida do resultado (CASELLA, ARAUJO e GIUDICI, 2003; SALDÍVAR et al., 2001).

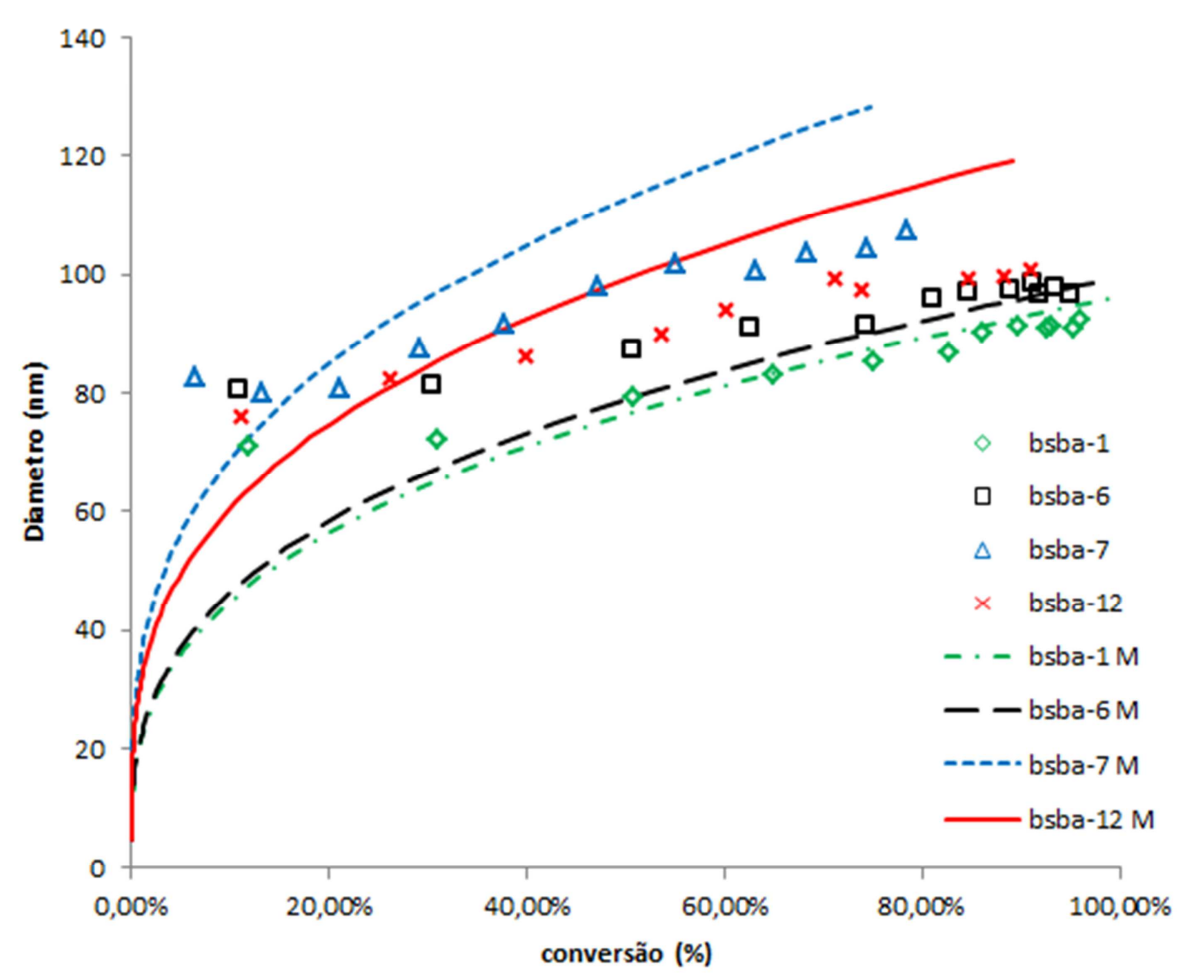

Figura 6.2 - Diâmetro das Partículas para os Experimentos BSBA-1, BSBA-6, BSBA-7 e BSBA-12

A Figura 6.3 mostra a evolução de conversão para as bateladas BSBA-2 e BSBA-4, onde a diferença das bateladas é somente na razão entre a massa de monômero e massa de água, apresentando uma boa aderência com relação aos resultados do modelo. Pode-se observar que a taxa de conversão é menor para uma maior concentração de monômero, pois a concentração de monômero nas partículas, onde ocorre a propagação, é dada pelo equilíbrio termodinâmico entre as fases gota, partícula e aquosa. Portanto, quanto maior a concentração, maior o 
tempo para que os monômeros sejam totalmente consumidos, sendo que os intervalos I, II e III, descritos na revisão bibliográfica, são as etapas físico-químicas que regem a polimerização em emulsão em batelada.

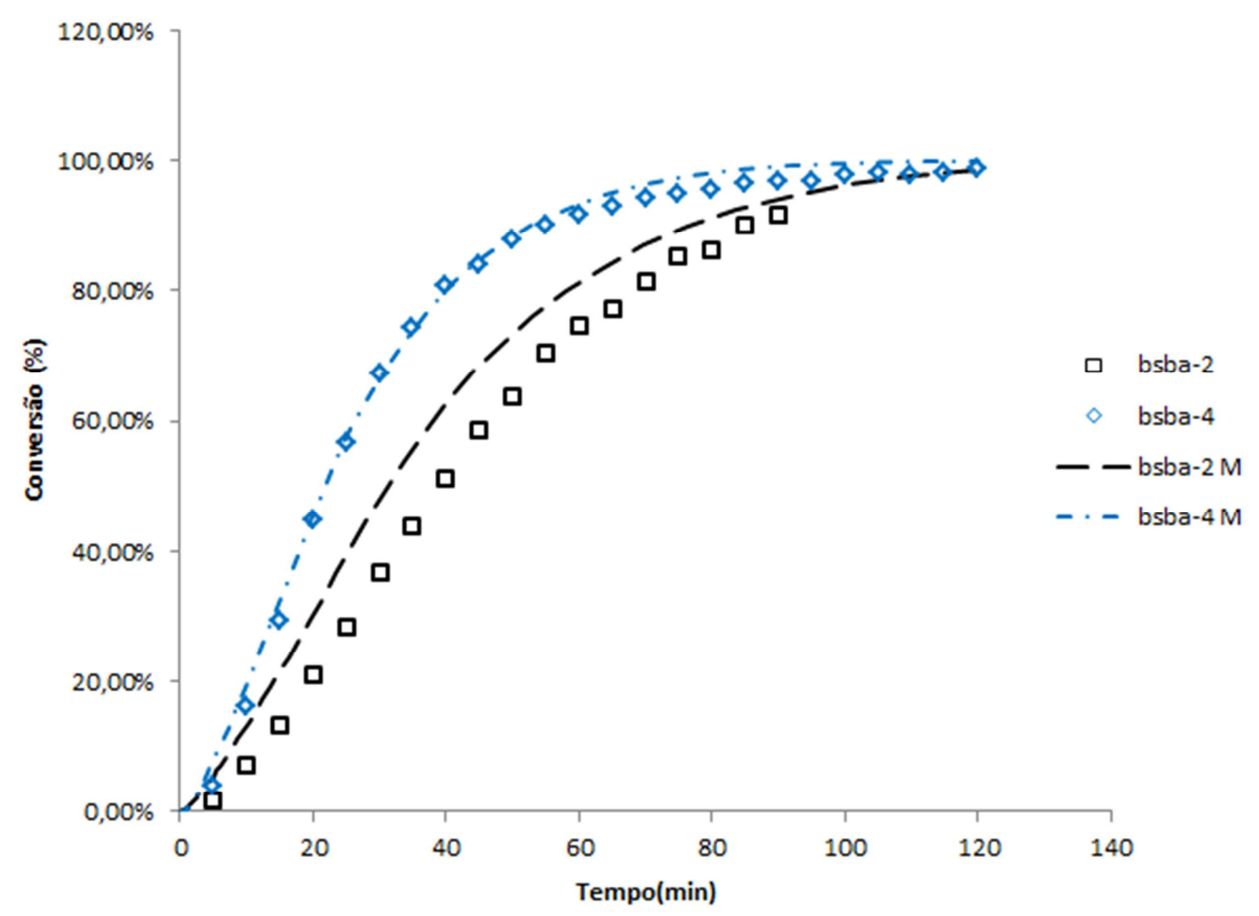

Figura 6.3- Conversão para os Experimentos BSBA-2 e BSBA-4

A Figura 6.4. mostra que o diâmetro médio das partículas é um pouco maior no caso de uma maior concentração de monômeros. Observa-se que os valores simulados para o número de partículas refletem o comportamento do perfil de diâmetros. 


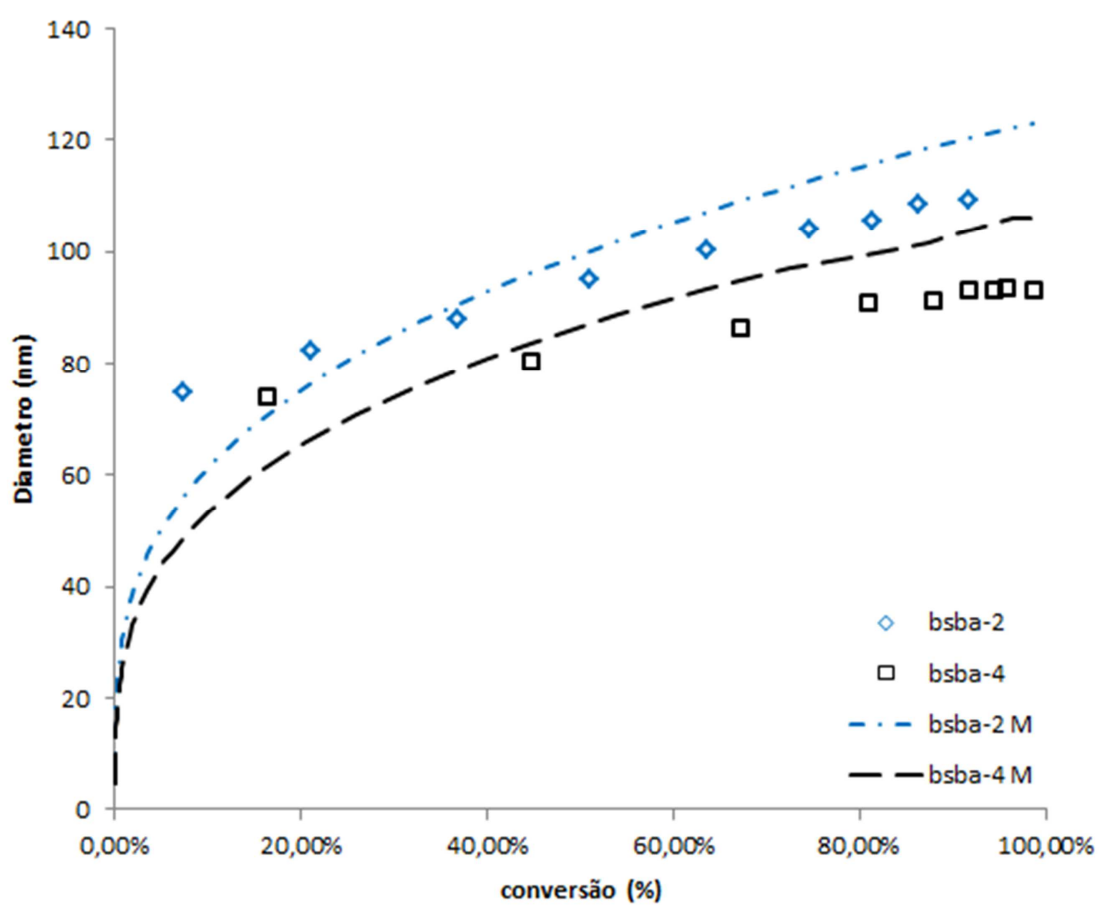

Figura 6.4 - Diâmetro Médio das Partículas Experimentos BSBA-2 e BSBA-4

Observa-se na Figura 6.5, onde só há somente alteração da temperatura da batelada entre os experimentos BSBA-4 e BSBA-12, que a taxa de conversão é maior com o aumento da temperatura. Neste ponto, é importante frisar que a temperatura é determinante para os fenômenos de propagação e de transferência de cadeia para monômero, pois são diretamente proporcionais as suas constantes. 


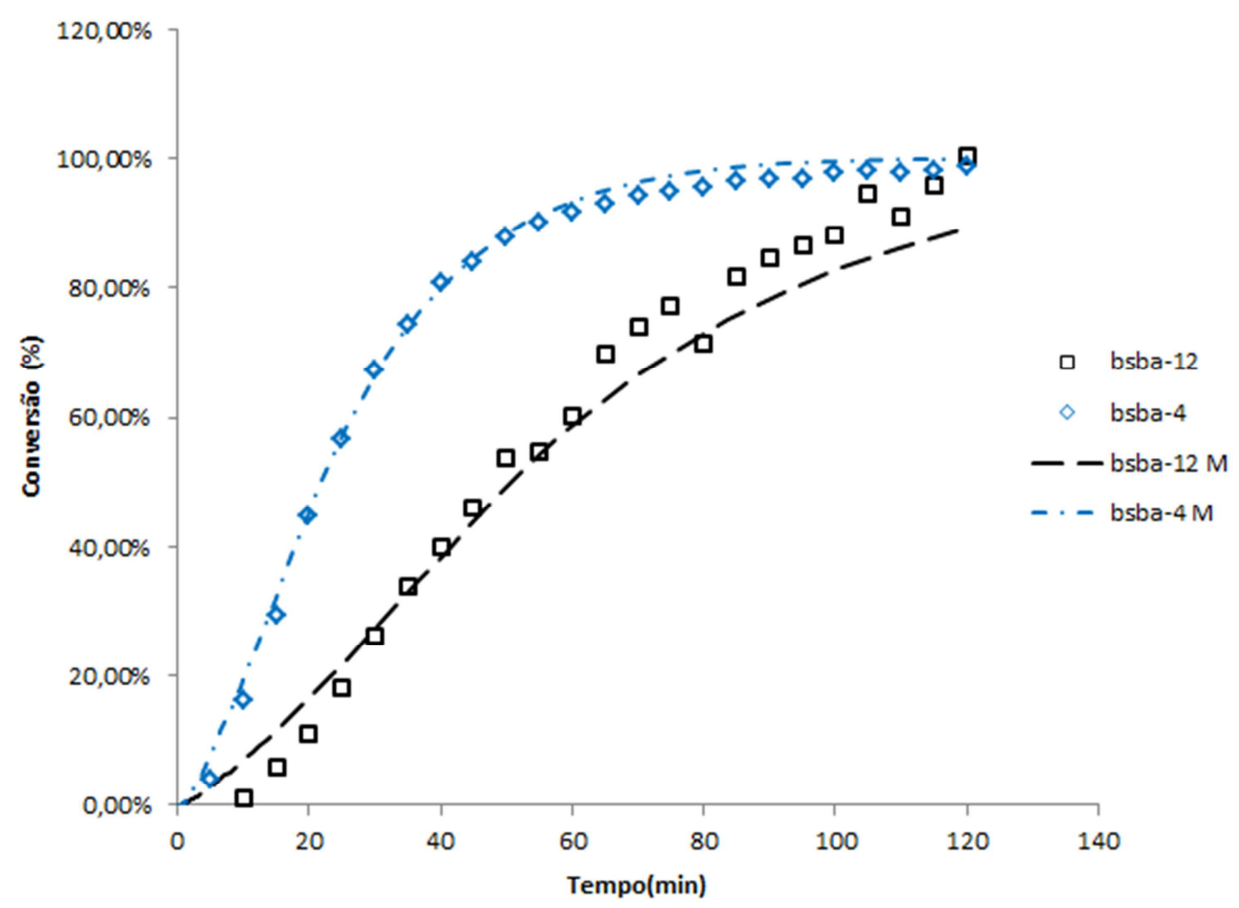

Figura 6.5 - Conversão para os Experimentos BSBA-4 e BSBA-12

A Figura 6.6 mostra o diâmetro das partículas em relação a conversão comparando os dois experimentos com o valores obtidos através da modelagem.

$\mathrm{O}$ ajuste destas curvas aos dados experimentais não se mostrou adequada, pois nas duas curvas a distância entre os pontos experimentais e o modelo é significativa. Os ajustes dos parâmetros $f_{\text {ke }}$ e $f_{\text {kemic }}$ foram ajustados por tentativa e erro. O ajuste dos parâmetros através de técnicas de otimização podem melhorar estes resultados, porém não foram acoplados a este modelo de simulação. Possíveis erros nas medições dos resultados experimentais não podem ser explicados pelo ajuste destes parâmetros.

Estes parâmetros também podem também sofrer alteração em relação à temperatura do processo, pois influenciam diretamente a taxa de captura de radicais pelas partículas e pelas micelas (CASELLA, ARAUJO e GIUDICI, 2003). 


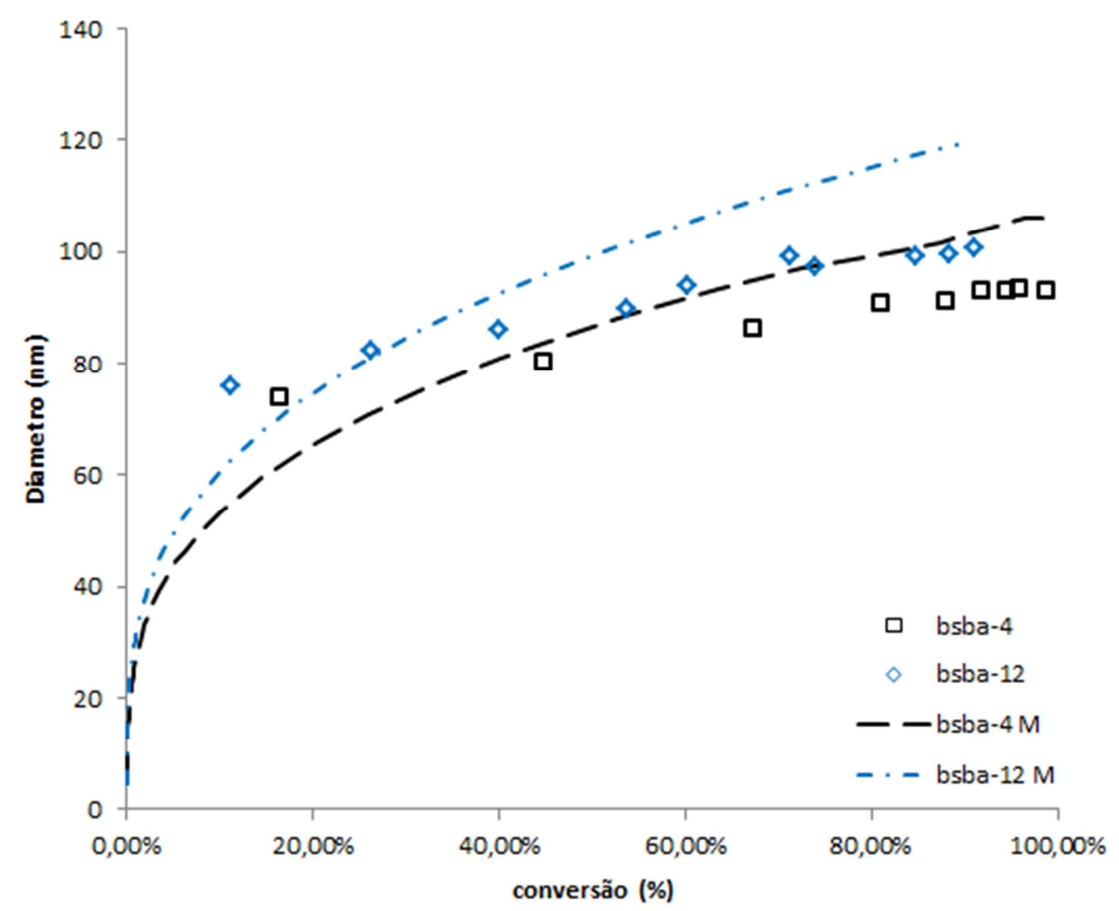

Figura 6.6 - Diâmetro Médio das Partículas Experimentos BSBA-4 e BSBA-12

Todos os experimentos foram modelados utilizando a modelagem proposta, entretanto observou-se que para experimentos com alto teor de acrilato de butila (BSBA-13 e BSBA-5), os resultados da modelagem ficam muito aquém dos erros aceitáveis. A Figura 6.7 mostra diferença entre os dados experimentais e o modelo.

Este fenômeno provém do fato de que o acrilato de butila tem a sua taxa de terminação alterada dependo das condições operacionais. Há uma intensificação deste efeito com o aumento da concentração deste monômero devido a efeitos de crosslinking (SALDíVAR et al., 2001). Os ajustes dos parâmetros relacionados ao efeito gel, que interferem diretamente a taxa de terminação, podem levar a uma boa aderência entre os dados experimentais para os casos de alta concentração de acrilato de butila no reator.

Este ajuste também pode ser feito diretamente na constante de propagação do acrilato de butila, porém não se considera adequado tal ajuste na abordagem adotada. Medições precisas das constantes de propagação dos monômeros utilizados em função da temperatura podem ser encontradas na literatura científica, sendo que alterações nestes valores, com intuito de melhorar ajustes dos resultados, podem deixar o modelo útil somente para os casos particulares. 


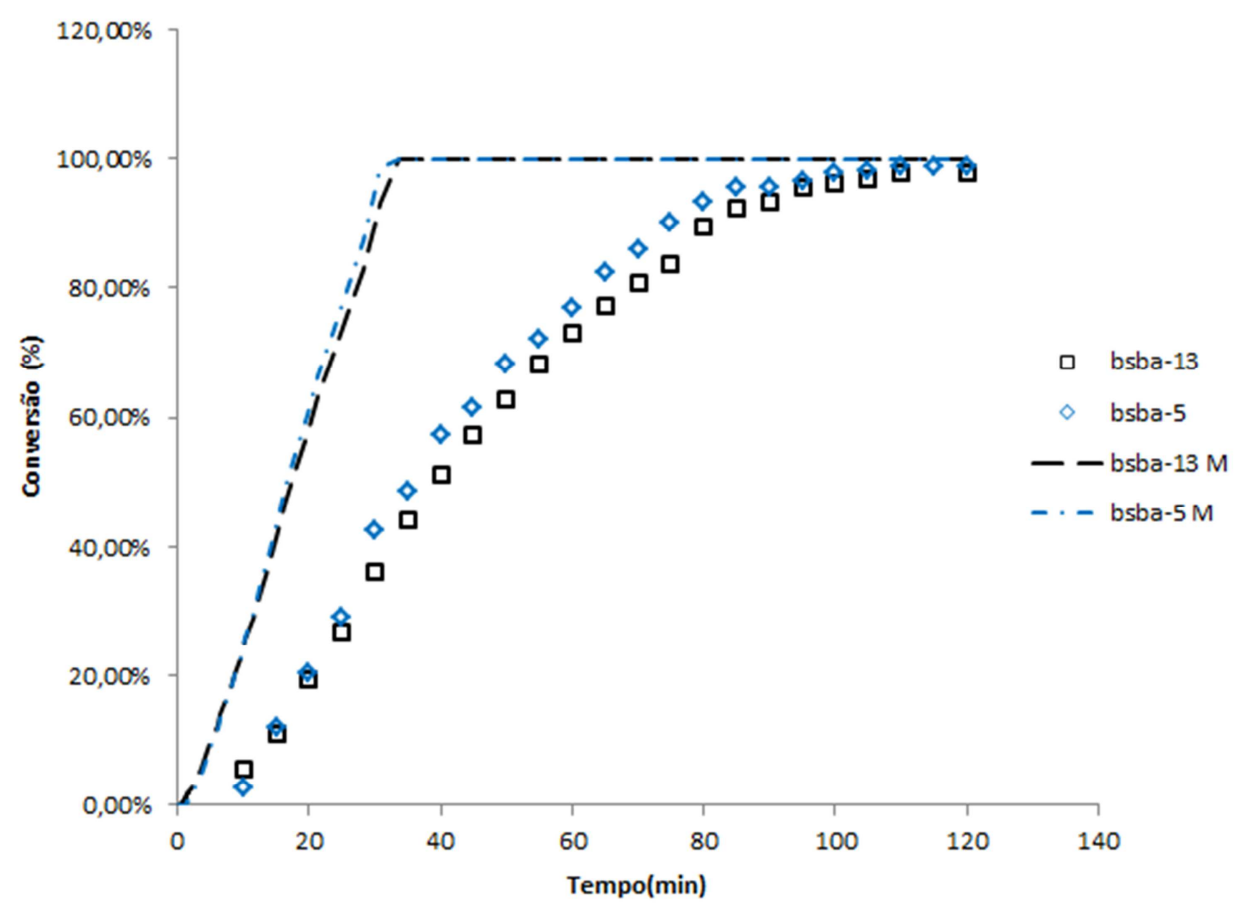

Figura 6.7 - Conversão para os Experimentos BSBA-5 e BSBA-13

\subsection{Experimentos (MARINANGELO, 2010)}

A Tabela 6.2 mostra os parâmetros $f_{k e}$ e $f_{\text {kemic }}$ ajustados para que os dados experimentais tivessem melhor aderência com os resultados do experimento AS-23. Este experimento foi escolhido, pois dentre os ensaios realizados pelo autor, apresenta uma gama de resultados mais completa e não há adição de um shot de emulsificante durante o processo de copolimerização. Este shot de emulsificante foi utilizado para aumentar a concentração de partículas no látex final nos ensaios realizados em 2010. Os resultados do modelo construído podem ser visualizados em uma série de gráficos e comparados aos valores experimentais.

Tabela 6.2 - Parâmetros estimados na modelagem (MARINANGELO, 2010)

\begin{tabular}{|c|c|c|c|}
\hline$f_{k e}$ & $f_{\text {kemic }}$ & ${ }^{\star} \mathrm{CMC}\left(\mathrm{kmol} / \mathrm{m}^{3}\right)$ & $\begin{array}{l}{ }^{*} a_{e}=a_{s} \cdot N_{A}{ }^{-1} \\
\left(\mathrm{~m}^{2} / \text { molécula }\right)\end{array}$ \\
\hline $1,5 \cdot 10^{-1}$ & $0,25 \cdot 10^{-8}$ & $2,679 \cdot 10^{-4}$ & $5,7 \cdot 10^{-19}$ \\
\hline
\end{tabular}

*parâmetros disponíveis em literatura 


\subsubsection{Experimento AS-23}

O diâmetro das partículas da simulação bem como nos dados experimentais são apresentados na Figura 6.8. Observa-se uma boa aderência entre os valores experimentais e os de simulação, apesar do patamar acima dos valores experimentais quando se compara com o número de partículas no reator, como apresentado na Figura 6.9.

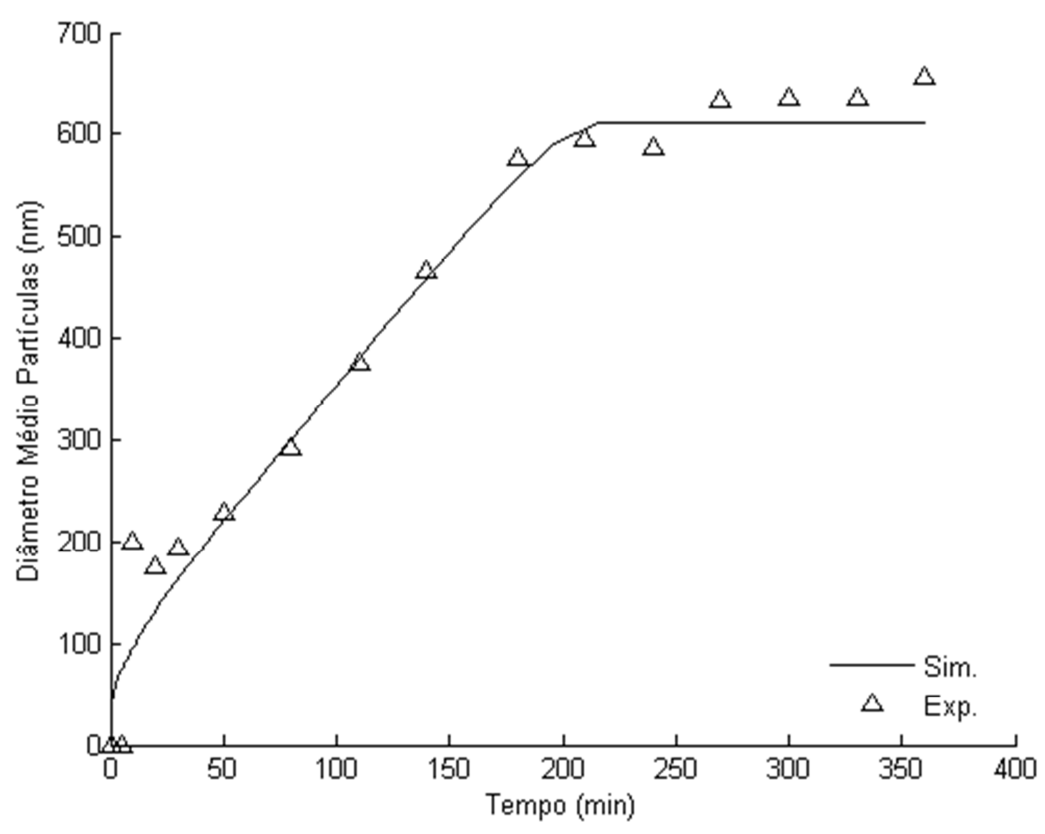

Figura 6.8 - Diâmetro médio das partículas ao longo do tempo - AS23

A Figura 6.9 mostra claramente a rápida nucleação de partículas em uma primeira fase da polimerização, chegando a uma concentração máxima de partículas tempo de reação em torno de 50 minutos. Neste momento todas as partículas provenientes da nucleação heterogênea foram formadas com o consumo total das micelas do meio. Após este instante, observa-se decrescimento da concentração de partículas que é explicado pela continua alimentação de pré-emulsão no reator até o tempo de batelada de 180 minutos. Observa-se o fenômeno de crescimento das partículas até o final da polimerização. O pequeno crescimento após o fim da 
alimentação é proveniente da nucleação homogênea que é predominante nesta etapa da reação.

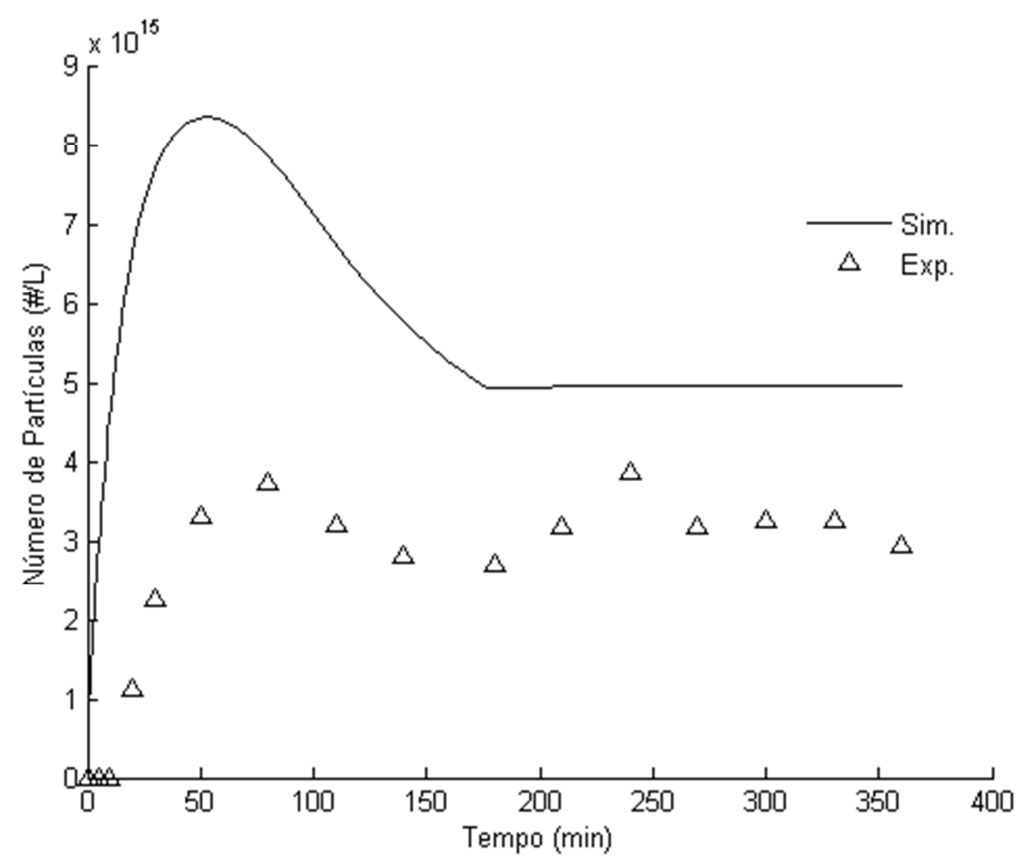

Figura 6.9 - Número de Partículas por Litro de Emulsão - AS23

Apesar da ordem de grandeza para o número de partículas por litro de emulsão ser a mesma dos dados experimentais, o patamar esta acima do encontrado experimentalmente. Este fenômeno pode ser explicado pelo ajuste não otimizado de parâmetros do modelo ou mesmo por imprecisões nas medidas experimentais.

Observa-se que o formato da curva apresentada pelos dados experimentais e aquela apresentada pelo modelo são semelhantes, excluindo-se o ponto que está em torno do tempo de reação de 250 minutos, que pode ser tratado como um outlier.

A evolução da fração de polímeros no reator, apresentado na Figura 6.10, cresce um pouco mais rapidamente que o medido em laboratório. Este comportamento era esperado, devido ao resultado de simulação para o número de partículas por litro de emulsão estar um pouco acima do experimental e o resultado de simulação para o diâmetro médio das partículas ficar bastante aderente aos experimentos. 


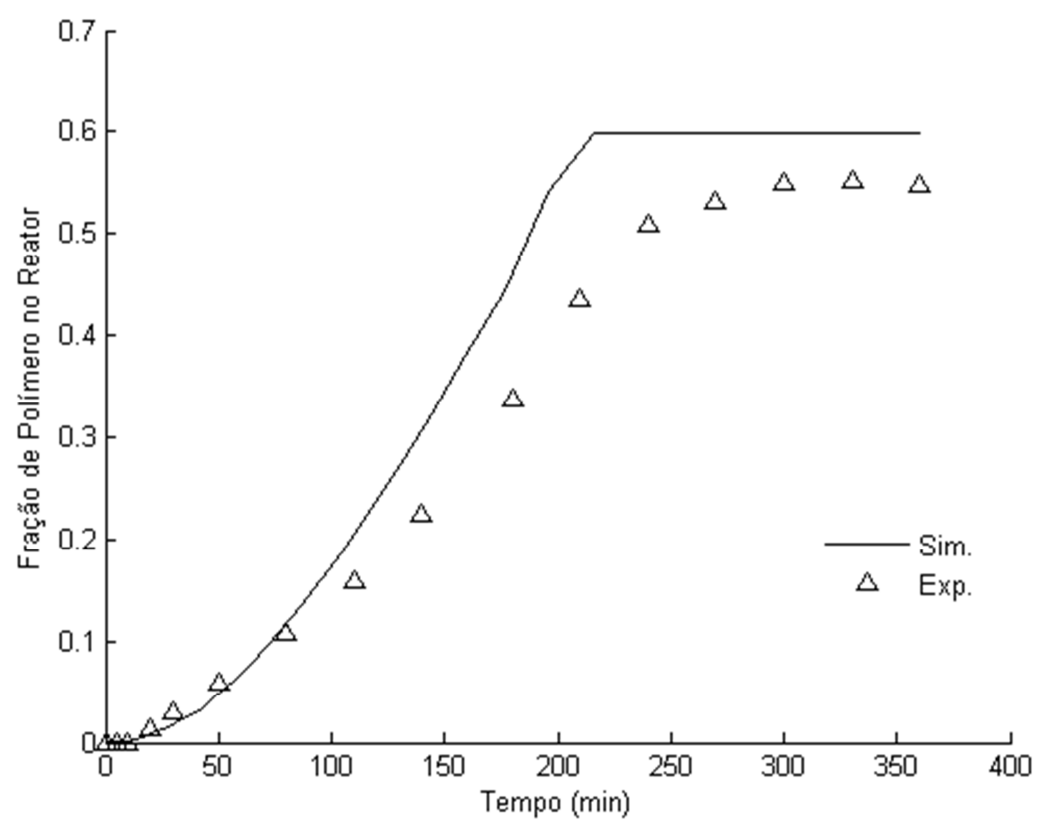

Figura 6.10 - Fração de Polímero no Reator - AS23

Observa-se que a fração de polímeros para estes experimentos chegou a um patamar de $55 \%$ enquanto que o modelo apresentou um patamar de $60 \%$ no final da reação, esta diferença pode ser atribuída à imprecisão na coleta dos dados experimentais. Ressalta-se que a fração de polímeros no reator tem uma relação direta com a conversão.

O perfil apresentado na Figura 6.11 para o número médio de radicais por partículas tanto para o experimento quanto para o modelo, apresenta um máximo no em torno de 210 minutos de batelada, onde a conversão está muito próxima do máximo e ocorre o final da alimentação. O perfil modelado mostra-se aderente no início e no final da batelada, porém há uma aceleração em torno de 150 minutos de batelada. Observa-se o mesmo crescimento e o decréscimo do número de radicais por partícula nos dados experimentais ao longo do tempo, porém em um patamar muito menor quando comparado aos resultados do modelo. 


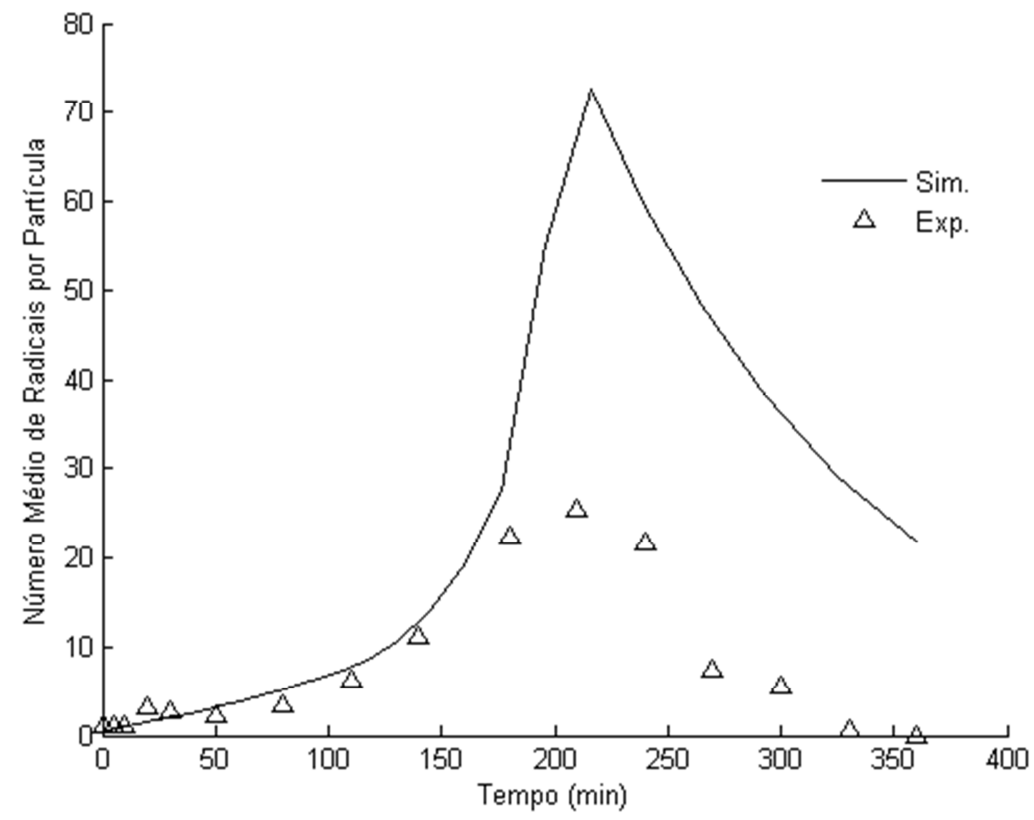

Figura 6.11 - Número de médio de radicais por partícula - AS23

A determinação do número de radicais por partícula experimentalmente incorre em muitos erros, porém é parte fundamental para o ajuste dos parâmetros. No âmbito da modelagem, esta é uma das equações diferenciais (Equação (4.13)) fundamentais para o bom entendimento do fenômeno. Apesar das curvas não coincidirem, o perfil é similar.

A Figura 6.12, Figura 6.13 e a Figura 6.14 mostram os resultados da solução do balanço populacional para o diâmetro das partículas, comparando os dados experimentais da fração volumétrica de partículas com o modelo ao longo do tempo de reação. 


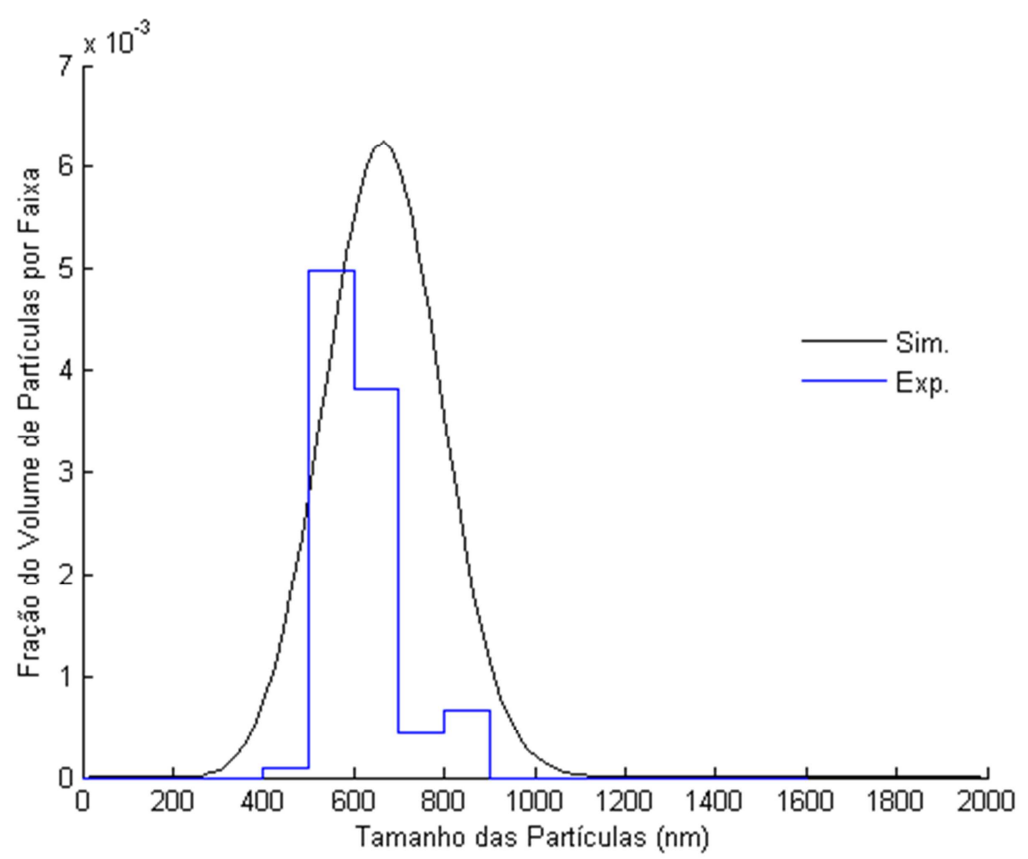

Figura 6.12 - Distribuição da fração volumétrica das partículas para o último instante de simulação AS23

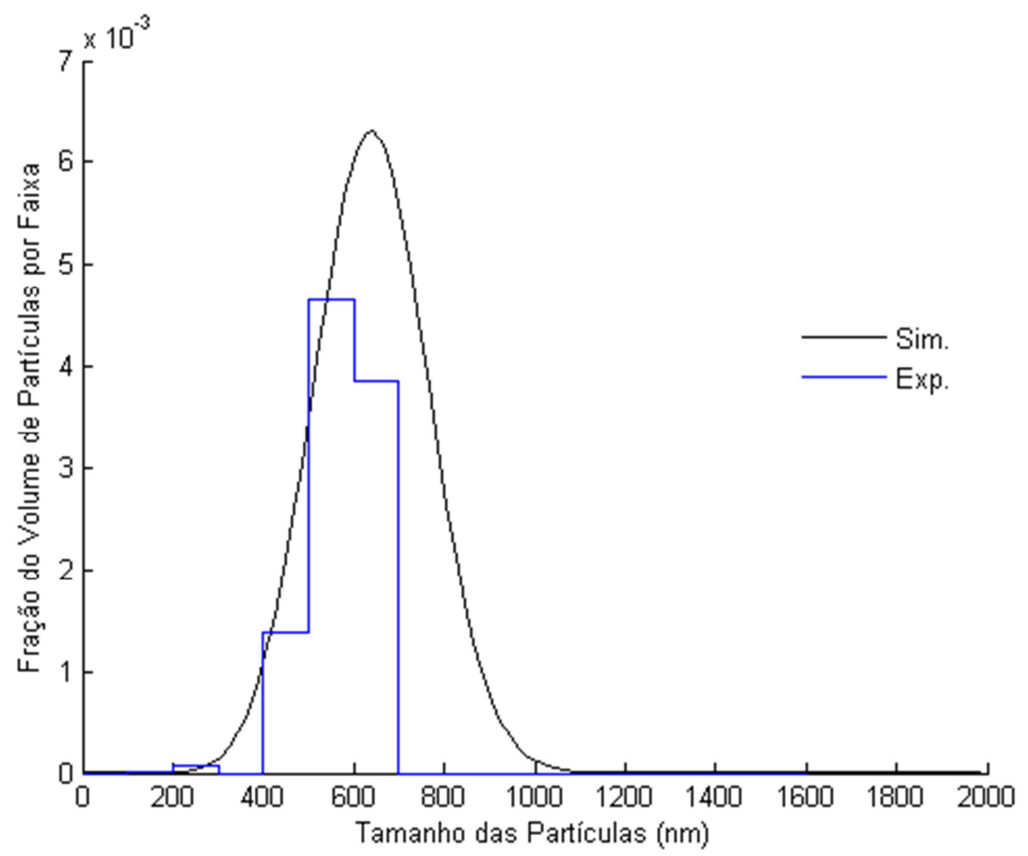

Figura 6.13 - Distribuição da fração volumétrica das partículas para o t=180 min de simulação - AS23 


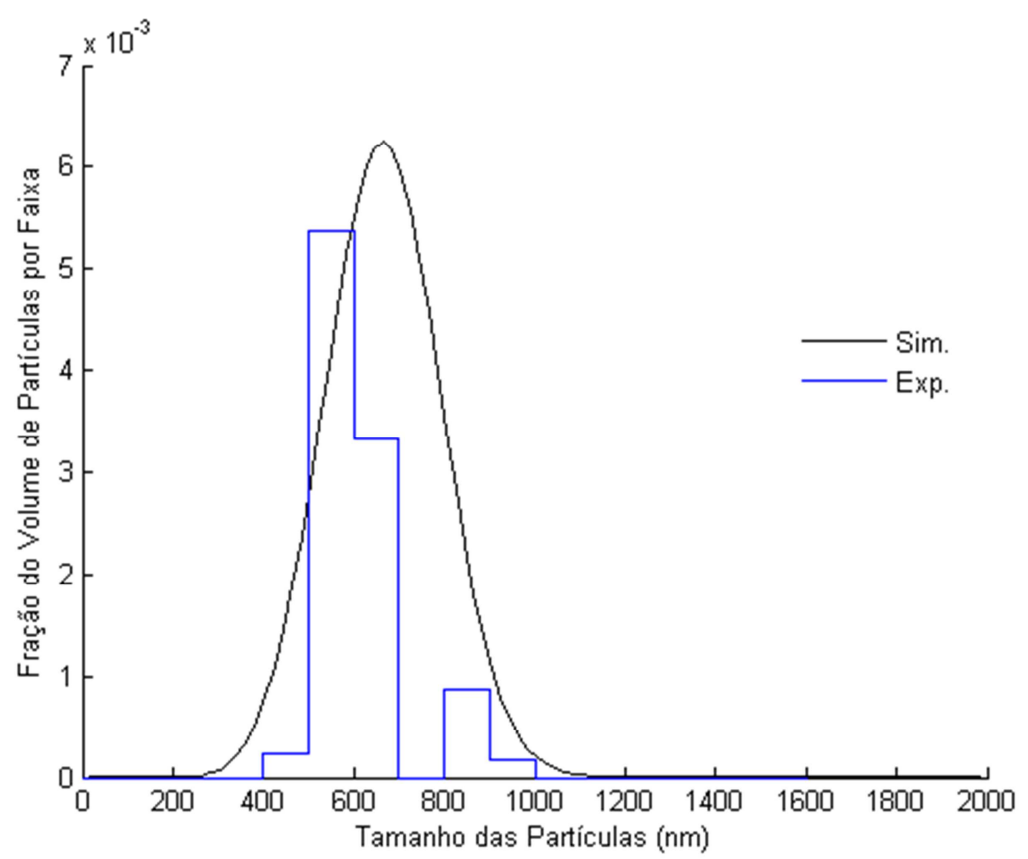

Figura 6.14 - Distribuição da fração volumétrica das partículas para o t=270 min de simulação - AS23

Mostra-se uma boa aderência do modelo com a fração volumétrica de partículas com os resultados experimentais. Entretanto, visualmente pode-se verificar um pequeno viés à direita dos resultados da simulação para os instantes simulados quando comparados com os dados experimentais.

A Figura 6.15 ilustra a evolução da distribuição do número de partículas ao longo do tempo, resultado do modelo em cada passo de integração. Pode-se observar que há um crescimento do diâmetro médio e da dispersão da distribuição ao longo do tempo. 


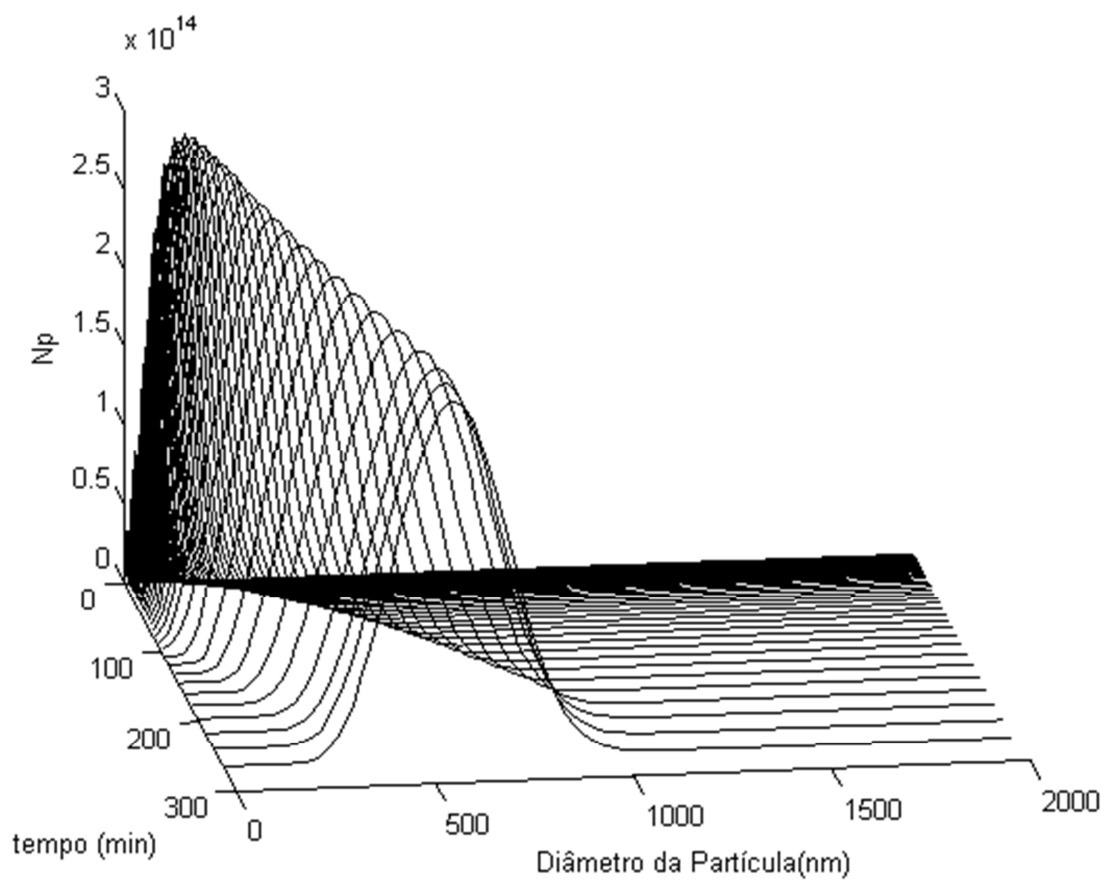

Figura 6.15 - Evolução da distribuição de tamanho de partículas ao longo do tempo.

\subsubsection{Experimento AS-22}

O diâmetro médio das partículas da simulação bem como os dados experimentais são apresentados na Figura 6.16. Observa-se que os dados experimentais estão com um patamar acima daqueles encontrados pela simulação. Aplicaram-se os mesmos parâmetros ajustados ao experimento AS-23. 


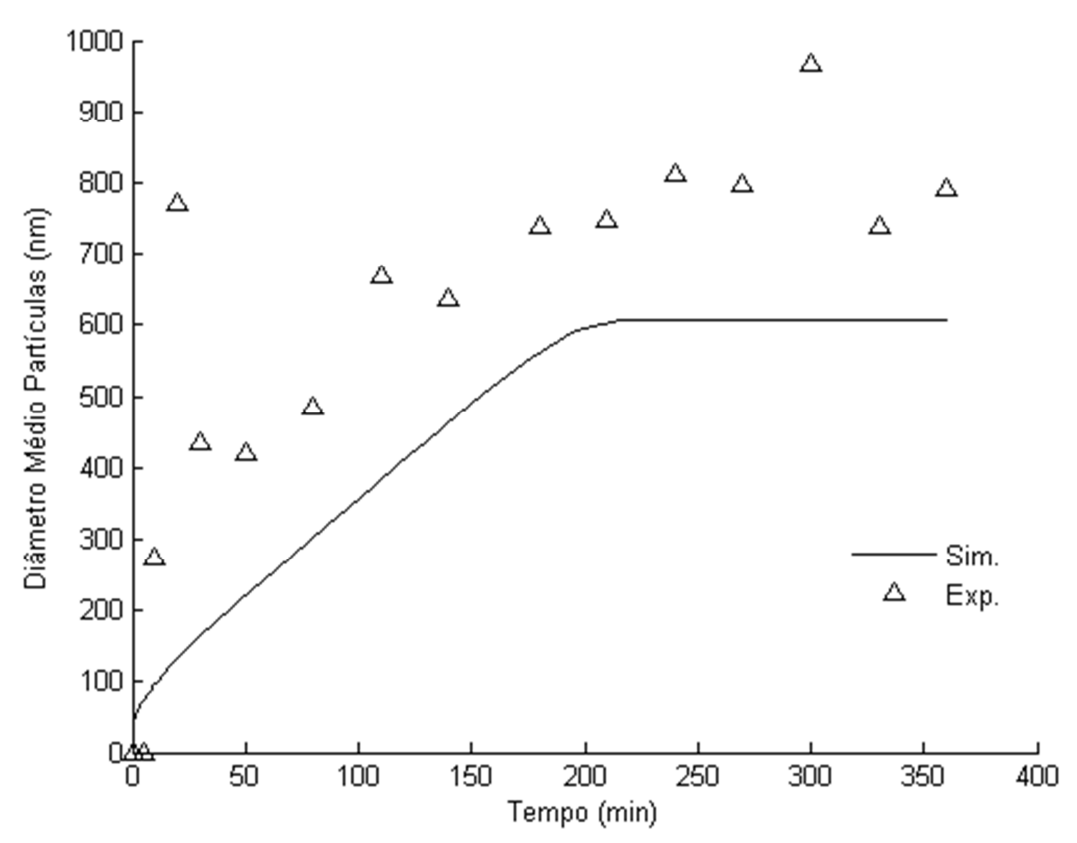

Figura 6.16 - Diâmetro médio das partículas ao longo do tempo - AS22

Na Figura 6.17, observa-se um patamar acima do desejado para o número de partículas por litro de emulsão. A influência do ajuste de parâmetro $f_{\text {kemic }}$ pode explicar tal diferença, sendo que a calibragem do mesmo se torna imprescindível para uma boa aderência do modelo aos dados experimentais. Como o patamar modelado da concentração de partículas do experimento AS-23 estava acima dos dados observados, apesar da mesma ordem de grandeza, os valores modelados do experimento AS-22 se comportaram de forma semelhante e também se apresentaram acima que os dados experimentais. Vale ressaltar que o número de partículas é calculado a partir do diâmetro médio das partículas e da conversão, o que pode levar a imprecisões nas medições experimentais. 


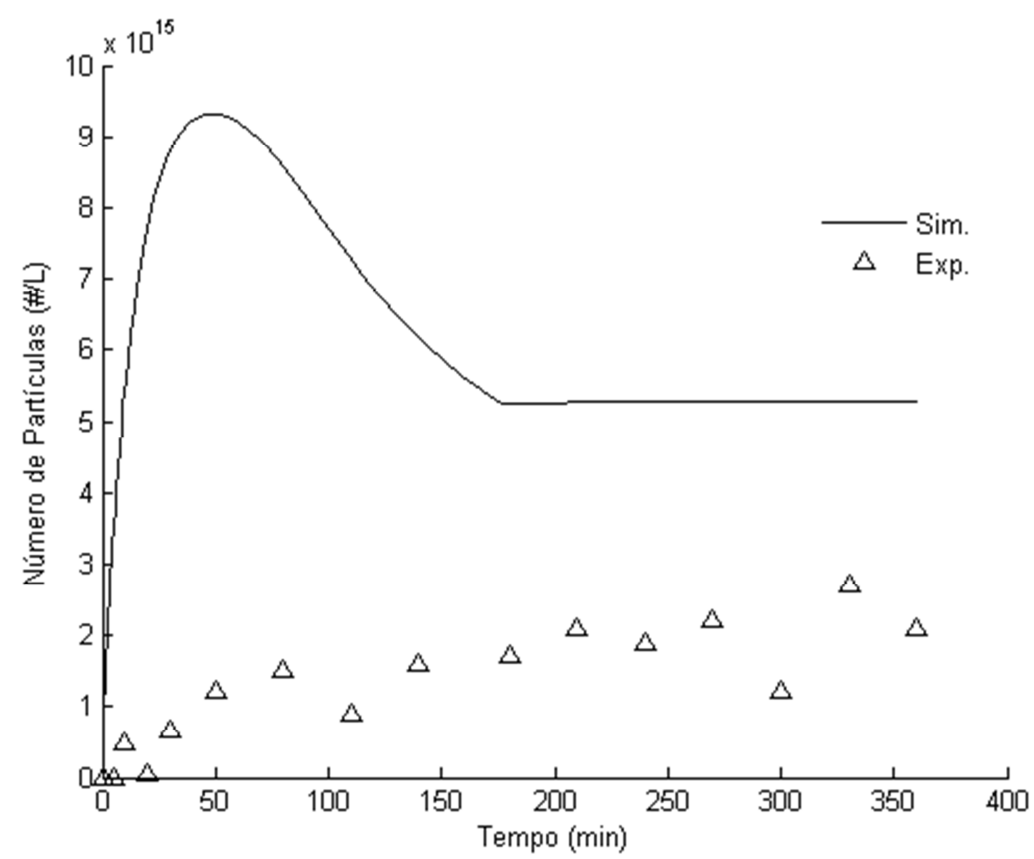

Figura 6.17 - Número de Partículas por Litro de Emulsão - AS22

Os valores experimentais para a fração de polímero no reator, Figura 6.18 , apresentam a mesma característica do experimento AS-23, sendo que o crescimento e o patamar modelado ficam ligeiramente acima dos dados experimentais.

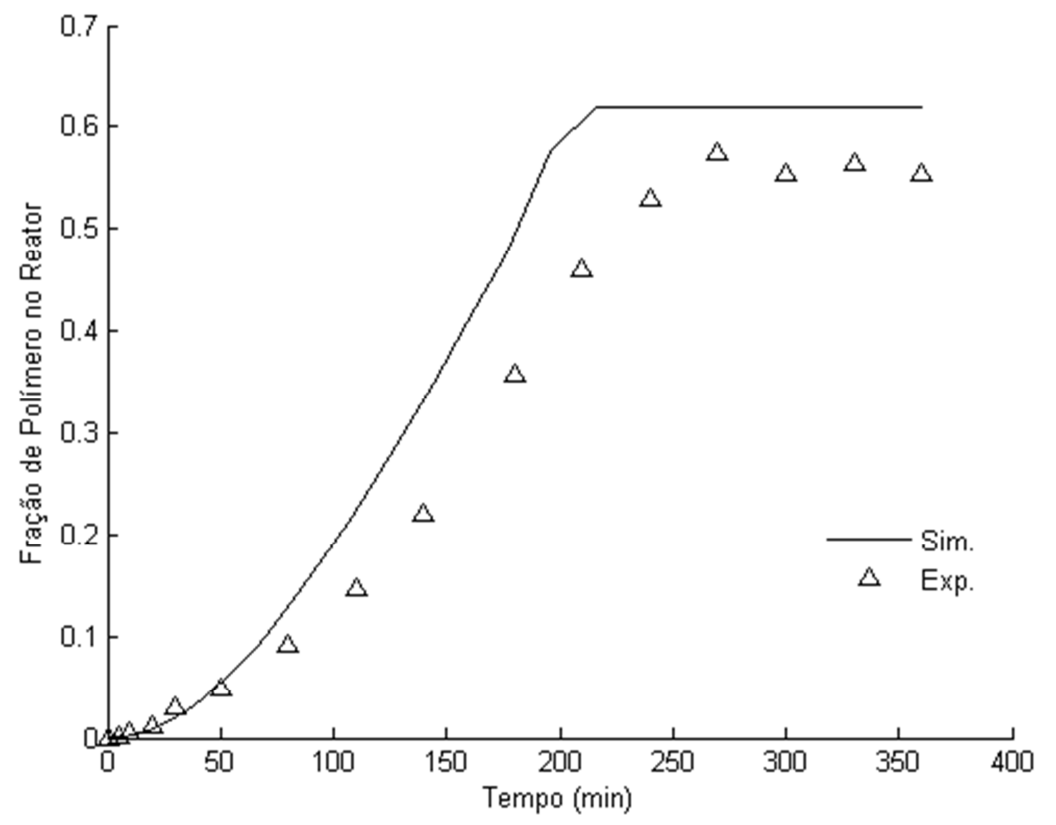

Figura 6.18 - Fração de Polímero no reator - AS22 
O número médio de radicais por partícula, Figura 6.19, mostra-se aderente aos resultados experimentais, apesar dos dados experimentais não mostrarem um perfil tão estável, sendo disperso. Neste experimento, não foram obtidos dados referentes à distribuição da fração volumétrica das partículas ou mesmo da massa molar média numérica e mássica.

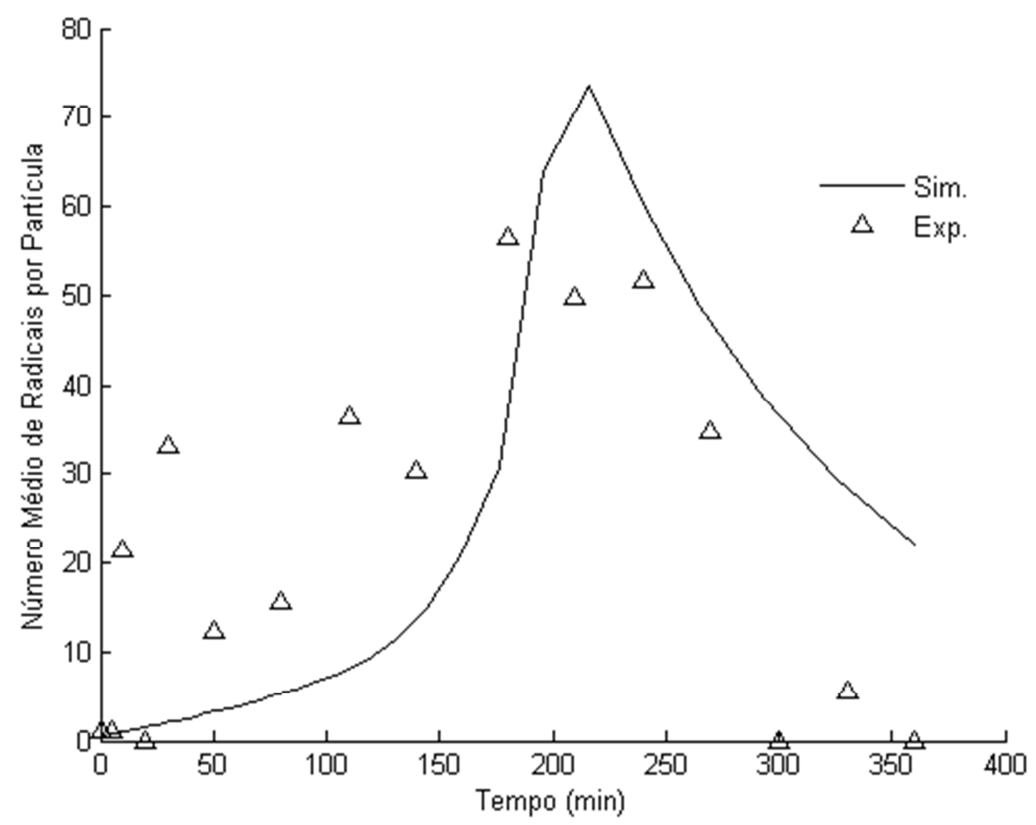

Figura 6.19 - Número de médio de radicais por partícula - AS22

A modelagem do processo de polimerização em emulsão é uma tarefa complexa, pois pequenas variações nos parâmetros de ajuste podem levar a resultados diferentes dos observados experimentalmente, mesmo para ensaios similares como AS-23 e AS-22. Contudo, a modelagem aqui apresentada, ajuda a entender e prever os fenômenos envolvidos.

\subsection{Experimentos (BENYAHIA et al., 2010)}

A Tabela 6.3 mostra os parâmetros ajustados aos dados experimentais comparando os resultados do modelo ao experimento R2. As condições operacionais deste experimento são apresentadas na Tabela 5.4. O valor de CMC não está disponível na bibliografia e foi arbitrado para o $\mathrm{CMC}$ do emulsificante 
dodecil sulfato de sódio, utilizado no primeiro conjunto de experimentos apresentado.

Tabela 6.3 - Parâmetros estimados na modelagem (BENYAHIA et al., 2010)

\begin{tabular}{|c|c|c|c|}
\hline$f_{k e}$ & $f_{\text {kemic }}$ & CMC $\left(\mathrm{kmol} / \mathrm{m}^{3}\right)$ & $\begin{array}{l}{ }^{*} a_{e}=a_{s} \cdot N_{A}{ }^{-1} \\
\left(\mathrm{~m}^{2} / \text { molécula }\right)\end{array}$ \\
\hline $0,25 \cdot 10^{-4}$ & $1 \cdot 10^{-7}$ & $2.43 \cdot 10^{-3}$ & $1,98 \cdot 10^{-18}$ \\
\hline
\end{tabular}

*parâmetros disponíveis em literatura

A Figura 6.20 mostra a conversão ao longo do tempo para os experimentos selecionados. Observa-se que o aumento da temperatura para os experimentos R3 e R4 propiciou um aumento na taxa de propagação, devido à dependência da mesma com a temperatura. Entretanto, a taxa de conversão do modelo está maior que a taxa de conversão dos experimentos, sendo pouco aderente.

A conversão depende exclusivamente da taxa de propagação, que para o caso do acrilato de butila apresenta uma ampla gama de valores na literatura, devido ao efeito que ocorrem na molécula durante a propagação, como o backbiting que faz com que os radicais formados por este fenômeno tenham uma reatividade menor que os radicais primários formados.

Exclusivamente para este caso foi incorporado à modelagem o efeito vítreo que atua sobre os valores da taxa de propagação (BENYAHIA et al., 2010).

$$
k_{p i j}=k_{p i j}^{0} \cdot \exp \left[-a_{i j}^{G l} \cdot\left(\phi_{p}^{p}-0,7\right)\right] \quad \phi_{p}^{p}>0,7
$$

onde, $k_{p i j}^{0}$ é o coeficiente de propagação na temperatura de reação e $a_{i j}^{G l}$ é uma constante que depende da combinação de monômeros $i$ e $j$. No caso da fração de polímeros da partícula ser menor que $70 \%$, a propagação mantém seu valor original.

Tabela 6.4 - Parâmetros utilizados para o cálculo do efeito vítreo: (BENYAHIA et al., 2010)

\begin{tabular}{c|c|c|c}
\hline$a_{A A}^{G l}$ & $a_{A B}^{G l}$ & $a_{B A}^{G l}$ & $a_{B B}^{G l}$ \\
\hline 17.13 & 5.73 & 5.73 & 5.73 \\
\hline
\end{tabular}




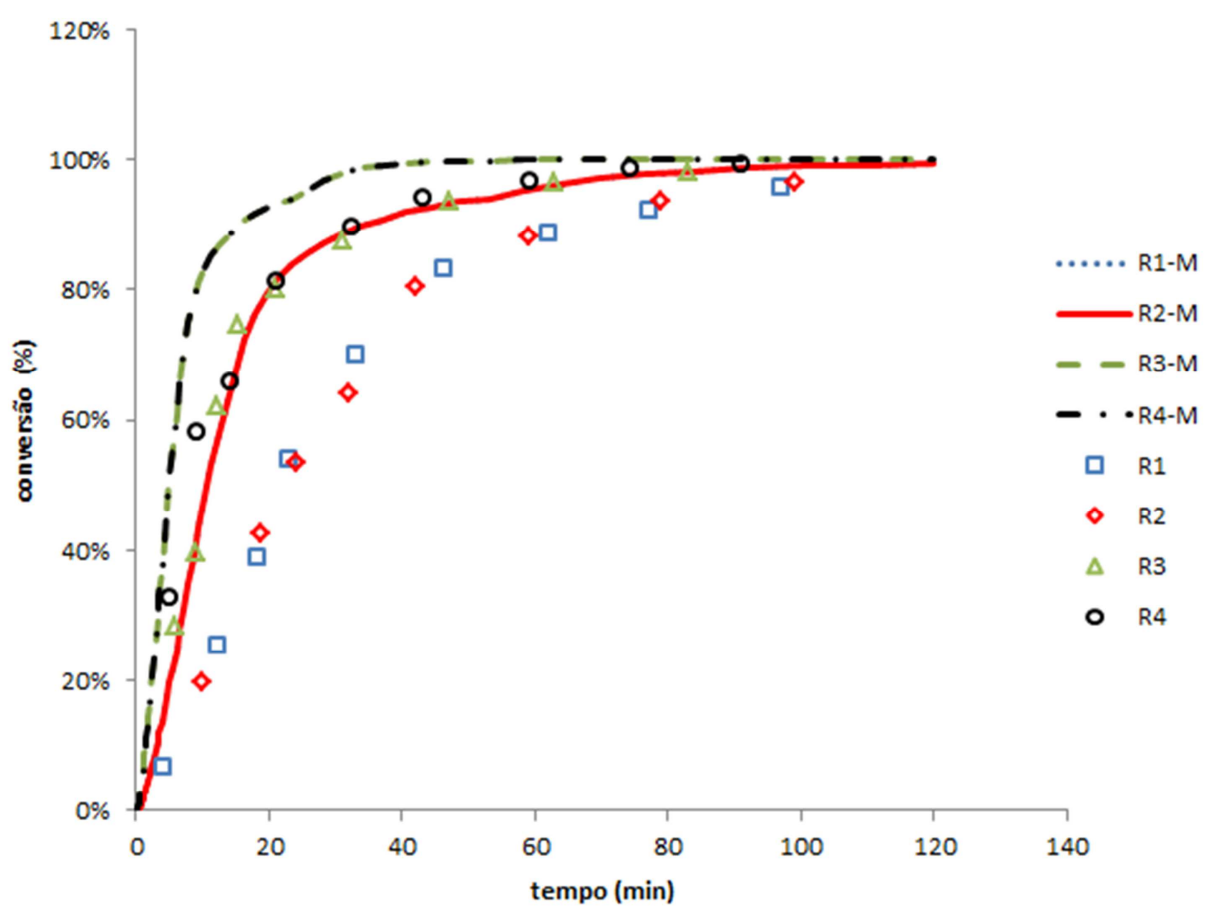

Figura 6.20 - Conversão ao longo do Tempo para os dados de R1 a R4

O perfil do diâmetro médio das partículas, apresentado na Figura 6.21, para todos os experimentos ficou muito aderente aos resultados obtidos através do modelo. $O$ experimento R4 foi o menos aderente, entretanto ainda apresenta a mesma ordem de grandeza dos experimentos assim com um perfil de curva similar aos obtidos em laboratório. 


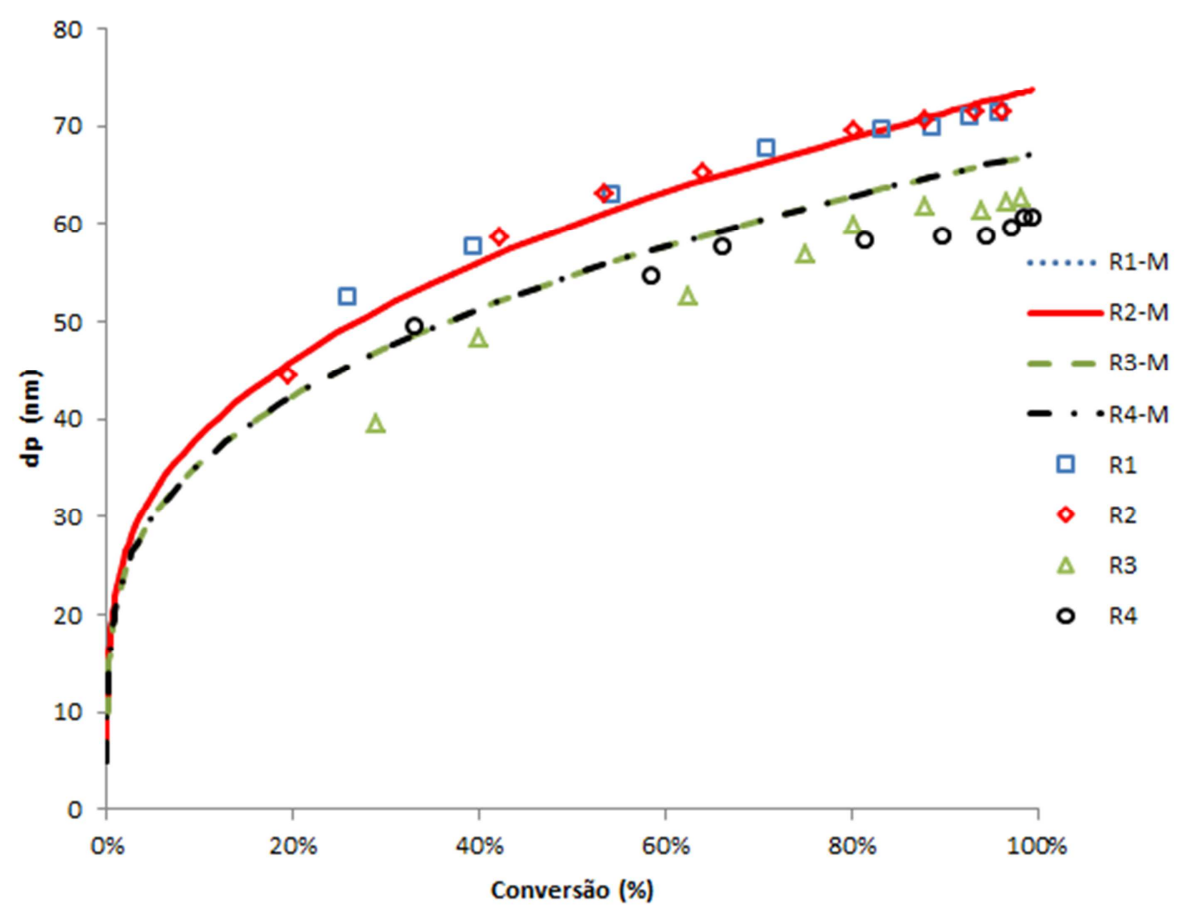

Figura 6.21 - Diâmetro Médio das Partículas para os experimentos de R1 a R4

A Figura 6.22 apresenta os valores obtidos no modelo para massa molar numérica média. Observa-se que os resultados obtidos têm a mesma ordem de grandeza do experimento e apresentam o mesmo perfil de decaimento ao logo da conversão, porém não estão tão aderentes aos valores experimentais.

Pode-se verificar que para o experimento $\mathrm{R} 1$ e $\mathrm{R} 3$, que foram conduzidos uma concentração menor de CTA, o modelo apresentou um patamar de massa molar maior, conforme esperado, pois o CTA controla a massa molar, e quanto maior a sua concentração menor é o tamanho da cadeia de polímero. Entretanto, considerando os experimentos, verifica-se que a diferença entre o patamar médio de R1 e R3 com o patamar médio de R2 e R4 é menor do que o apresentado pelo modelo. 


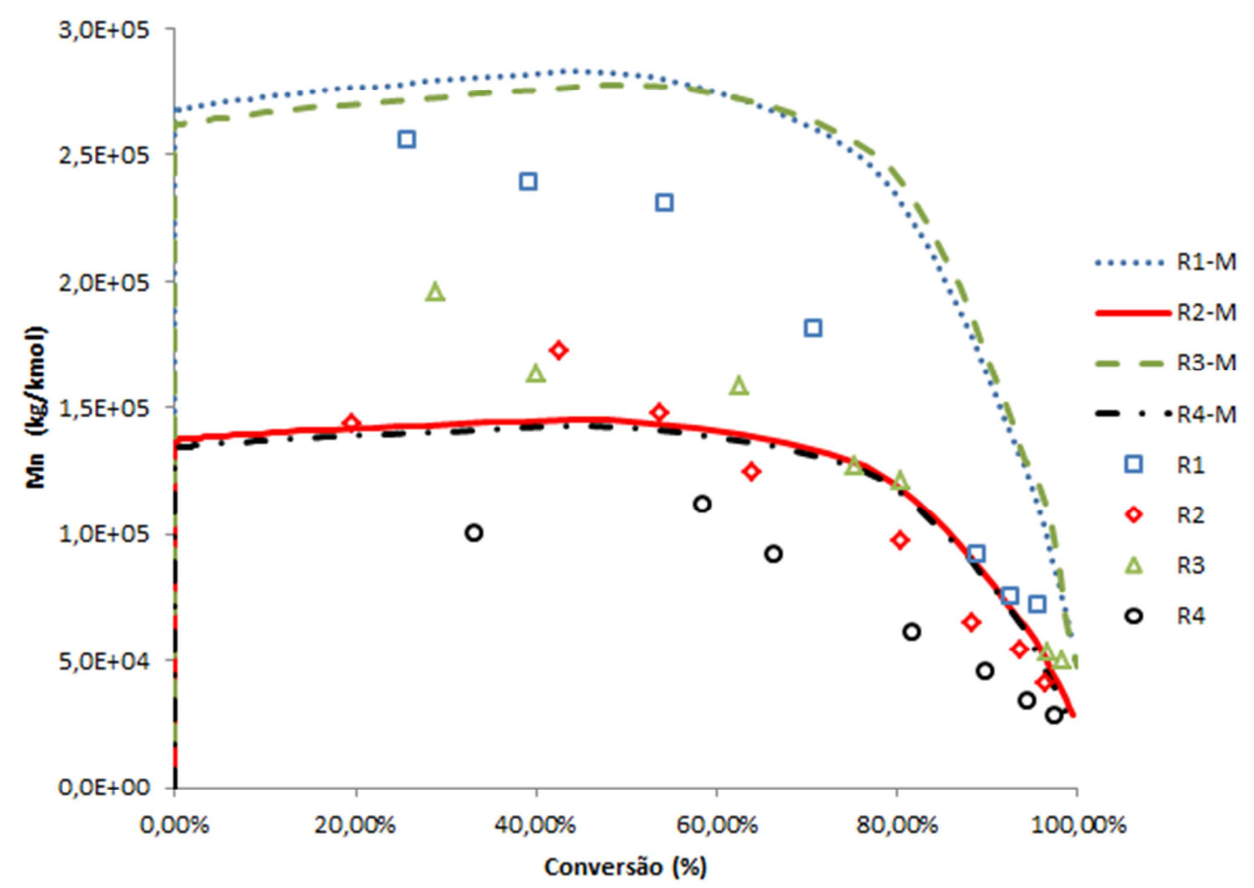

Figura 6.22 - Massa molar média numérica dos experimentos de R1 a R4.

O mesmo perfil é observado na Figura 6.23 para a massa molar média mássica, entretanto com aderência maior para experimentos $\mathrm{R} 1$ e R4. Vale ressaltar que a partir de dados da literatura (BENYAHIA et al., 2010), foi possível refinar os valores da constante de transferência de cadeia para CTA para que os valores calculados pelo modelo fossem aderentes aos valores experimentais. Para este modelo, refinamos os valores a partir do experimento R1 e o ajuste adotado foi de $40 \%$ do valor original. 


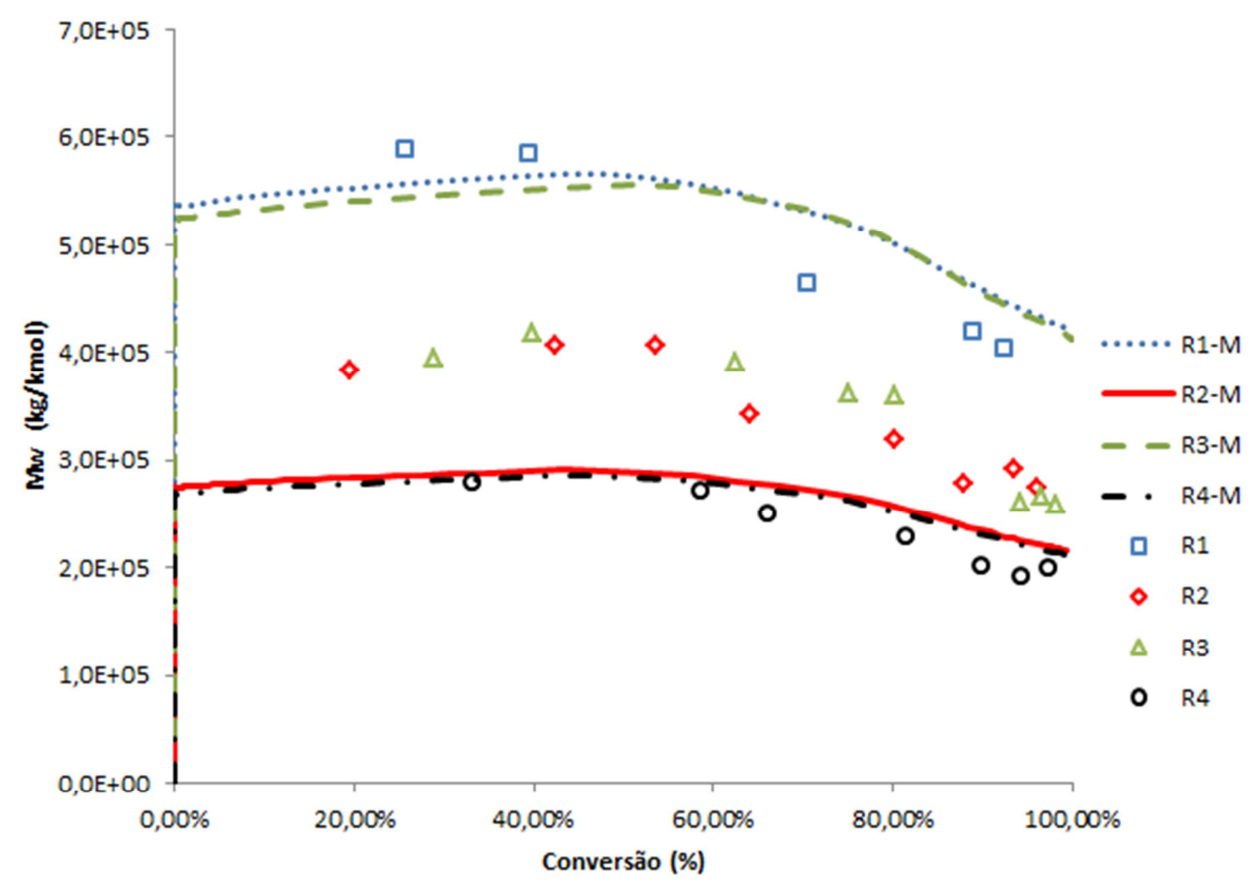

Figura 6.23 - Massa molar média mássica dos experimentos de R1 a R4.

O decrescimento observado nos dados experimentais e nos resultados de simulação das curvas de massa molar média numérica e mássica após altas taxa de conversão pode ser atribuído aos efeitos gel e vítreo que afetam respectivamente a taxa de terminação e propagação. 


\section{CONCLUSÕES E RECOMENDAÇÕES}

\subsection{Conclusões}

A modelagem computacional foi desenvolvida e aplicada ao processo de copolimerização em emulsão de acrilato de butila e estireno em reatores batelada e semi-batelada isotérmicos apresentando resultados aderentes aos dados experimentais adquiridos em literatura, exceto para casos em que a concentração de acrilato de butila era muito maior que a de estireno. Nestes casos, sabe-se que a conversão depende da taxa de propagação, que para o caso do acrilato de butila sofre efeitos de backbiting formando radicais menos reativos, o que propicia mudanças na taxa de propagação. Foi implementada uma solução numérica para o conjunto de equações algébrico-diferenciais que descrevem o problema. A solução do balanço populacional, tanto para a distribuição de tamanho de partículas quando para a distribuição média de radicais por partícula foi resolvida pelo método dos pivots fixos. A massa molar média numérica e mássica foi obtida através do método dos momentos.

Os resultados referentes à massa molar média numérica e mássica dependem da quantidade de agente de transferência de cadeia utilizada, que tem como função, controlar a massa molar média numérica e mássica dos polímeros formados. Portanto, a concentração de CTA foi tratada dentro do modelo, sendo que a difusão de CTA das gotas para a fase aquosa é o limitante do processo.

A construção do modelo de copolimerização em emulsão, utilizando o método dos pivots fixos, apresentou resultados aderentes aos dados experimentais. A solução das equações algébrico-diferenciais pelo método de diferenciação numérica (NDF) de ordem variada (ODE15S) acoplados ao modelo de resolução de equações algébricas não-lineares, reduziu significativamente o tempo computacional para a solução do problema e se mostrou robusta. A normalização das variáveis para a resolução das equações diferenciais, assim como, a separação das equações diferenciais que provém o número de partículas e a fração de partículas em cada classe garantiram a estabilidade do modelo e auxiliaram a reduzir esforço 
computacional. A opção de não-negativos para as variáveis das equações diferenciais também se mostrou adequada.

A estratégia de construir um modelo simplificado para a determinação dos fatores $f_{\text {ke }}$ e $f_{\text {kemic }}$, no intuito de utilizar posteriormente no modelo com balanço populacional foi uma estratégia acertada, pois reduz significativamente o tempo computacional para as rodadas de ajuste e propicia uma melhor estimativa para o modelo com balanço populacional.

Os experimentos em batelada (ARAÚJO, 1997) foram importantes para validação inicial, entendimento dos fenômenos envolvidos e limites do modelo construído. A gama de condições operacionais propiciou que diversos testes com o modelo pudessem ser realizados, sendo que para concentrações menores de acrilato de butila, o mesmo se mostrou bastante robusto e adequado.

O modelo construído teve boa aderência em relação à evolução do diâmetro médio das partículas ao longo do tempo considerando os experimentos AS22 e AS23 (MARINANGELO, 2010). A solução do balanço populacional, considerando a fração volumétrica de partículas, apresentou resultados aderentes quando comparados aos dados coletados em laboratório, mesmo quando se compara a evolução destas distribuições ao longo do tempo. O número de partículas por litro de emulsão no reator para os dois experimentos ficou com um patamar acima dos resultados experimentais, o que mostra uma oportunidade de refino no ajuste dos parâmetros.

Os resultados dos experimentos R1, R2, R3 e R4 (BENYAHIA et al., 2010) foram os escolhidos para a validação da massa molar média numérica e mássica. Vale ressaltar que os parâmetros do emulsificante usado não foram disponibilizados pelos autores e tiveram que ser estimados neste trabalho. Após ajustes nos coeficientes de propagação, foi possível uma aderência razoável aos dados experimentais para o cálculo da massa molar média numérica e mássica. $O$ diâmetro médio das partículas ficou aderente com os dados experimentais.

O modelo construído descreve adequadamente 0 processo de copolimerização em emulsão de acrilato de butila e estireno para sistemas batelada e semi-batelada isotérmicos, através da resolução dos balanços populacionais para tamanho de partículas e número de radicais por partícula, acoplado ao balanço de 
momentos que propicia estimação, com boa aderência, da massa molar média numérica e mássica ao longo da reação.

\subsection{Recomendações}

Recomenda-se para estudos futuros, realizar experimentos completos em batelada e semi-batelada isotérmicos para copolimerização em emulsão de acrilato de butila e estireno obtendo o diâmetro médio das partículas, número de partículas por litro de emulsão, número médio de radicais por partículas, distribuição de tamanho de partículas e a massa molar média numérica e mássica com o objetivo de validar o modelo completo desenvolvido.

Considera-se fundamental o acoplamento de um módulo de otimização para determinação dos parâmetros de ajuste $f_{k e}$ e $f_{\text {kemic }}$ para a solução do modelo simplificado, no intuito de melhorar a precisão dos ajustes executados manualmente através de tentativa e erro.

Sugere-se acoplar ao modelo as equações que consideram a coagulação de partículas, e planejar experimentos no intuito de conseguir determinar os parâmetros necessários para calibrar os termos de coagulação.

Uma evolução considerável na modelagem pode contemplar a evolução do modelo proposto para que além da distribuição de tamanho de partículas considere a distribuição de massas molares ao longo do tempo de reação. 


\section{REFERÊNCIAS}

ARAÚJO, O. Copolimerização e Terpolimerização em Emulsão: Trabalho Experimental e Estudo de Aspectos Relacionados a Modelagem Matemática do Processo. Tese de Doutorado, Universidade de São Paulo, Escola Politécnica, Brasil: [s.n.], 1997.

ARAÚJO, P. H. H.; GIUDICI, R. Optimization of semicontinuous emulsion polymerization reactions by IDP procedure with variable time intervals. Computers and Chemical Engineering, v.27, 2003. p.1345-1360.

ARAÚJO, P. H. H.; GIUDICI, R. Corrigendum to "Optimization of semicontinuos emuslion polymerization reactions by IDP procedure with variable time intervals". Computers and Chemical Engineering, v.28, 2004. p.575-578.

ARBINA, L. L.; BARANDIARAN, M. J.; GUGLIOTTA, L. M.; ASUA, J. M. Kinetics of the emulsion copolymerization of styrene and butyl acrylate. Polymer, v.38, 1997. p.143-148.

ASUA, J. M. A New model for radical desorption in emulsion polymerization. Macromolecules, v.36, 2003. p.6245-6251.

ASUA, J. M. Polymer reaction engineering. [S.I.]: Blackwell Publishing, 2007.

BENYAHIA, B.; LATIFI, M. A.; FONTEIX, C.; PLA, F.; NACEF, S. Emulsion copolymerization of styrene and butyl acrylate in the presence of a chain transfer agent. Chemical Engineering Science, v.65, 2010. p.850-869.

CARVALHO, A. C. S. M.; CHICOMA, D.; SAYER, C.; GIUDICI, R. Comparasion of vinyl acetate - butyl acrilate emulsion copolymerization conducted in a continuous pulse sieve plate column reactor and in a batch stirred tank reactor. Macromolecular Symposium, v.243, 2006. p.147-158.

CASELLA, E. L.; ARAUJO, O.; GIUDICI, R. Mathematical Modeling of Batch Emulsion Copolymerization Process. Polymer Reaction Engineering, v.11(4), 2003. p.869-910. 
CHERN, C. S. Emulsion polymerization mechanisms and kinetics. Progress in Pollymer Science, v.31, 2006. p.443-486.

DUBÉ, M. A.; SOARES, J. B. P.; PENLIDIS, A.; HAMIELEC, A. E. Mathematical modeling of multicomponent chain-growth polymerization in batch, semi batch and continuous reators: A review. Industrial \& Engineering Chemistry Research, v.36, 1997. p.966-1015.

FEIZ, S.; NAVARCHIAN, A. H. Emulsion polymerization of styrene: Simulation the effects of mixed ionic and non-ionic surfactant system in the presence of coagulation. Chemical Engineering Science, v.69, 2012. p.431-439.

FERRARI, J. Inferência por redes neurais da distribuição de tamanho de partícula em reações continuas de polimerização em emulsão. Tese de Doutorado, Universidade Federal de Santa Catarina, Brasil: [s.n.], 2014.

FORCADA, J.; ASUA, J. M. Modeling of unseeded emulsion copolymerization of styrene and methyl methacrylate. Journal of Polymer Science Part A: Polymer Chemistry, v.28, 1990. p.987-1009.

FRIIS, N.; HAMIELEC, A. E. Gel effect in emulsion polymerization of vynil monomers. Emulsion Polymerization. ACS Symposium Series, v.24, 1976. p.82-91.

GAO, J.; PENLIDIS, A. Mathematical modeling and computer simulator/database for emulsion polymerizations. Progress in Polymer Science, v.27, 2002. p.403-535.

GILBERT, R. G. Emulsion Polymerization. A Mechanistic Approach. San Diego: Academic Press, 1995.

GINSBURGER, E.; PLA, F.; FONTEIX, C.; HOPPE, S.; MASSEBEUF, S.; HOBBES, P.; SWAELS, P. Modelling and simulation of batch and semi-batch emulsion copolymerization of styrene and butyl acrylate. Chemical Engineerig Science, v.58, 2003. p.4493-4514.

GRAND VIEW RESEARCH. Emulsion Polymer Market Analysis By Product, by Application and Segment Forecasts To 2020, 2015. Disponivel em: <http://www.grandviewresearch.com/industry-analysis/emulsion-polymer-market>. 
HAMZEHLOU, S.; REYES, Y.; HUTCHINSON, R.; LEIZA, J. R. Copolymerization of n-Butyl Acrilate and Styrene: Terminal vs Penultimate Model. Macromolecular Chemistry and Physics, v.215, 2014. p.1668-1678.

HARKINS, W. D. A General Theory of the Mechanism of Emulsion Polymerization. Journal of American Chemical Society, v.69, 1947. p.1428-1444.

HIROTA, W. D. Avaliação de técnicas calorimétricas aplicadas ao monitoramento de processos químicos. Tese de Doutorado, Universidade de São Paulo, Escola Politécnica, Brasil: [s.n.], 2009.

HOSSEINI, A.; BOUASWAIG, A. E.; SEBASTIAN, E. Comparison of classical population balance models of emulsion polymerization with experimental results and a stochastic extension. Chemical Engineering Science, v.72, 2012. p.179-194.

HVALA, N.; ALLER, F.; MITEVA, T.; KUKANJA, N. Modeling, simulation and control of an industrial, semi-batch, emulsion-polymerization reactor. Computers \& Chemical Engineering, v.35, 2011. p.2066-2080.

IMMANUEL, C. D.; CORDEIRO, C. F.; SUNDARAM, S. S.; MEADOWS, E. S.; CROWLEY, T. J.; DOYLE III, F. J. Modeling of Particle Size Distribution in Emulsion Co-polymerization: Comparison with Experimental Data and Parametric Sensitivity Studies. Computers and Chemical Engineering, v.26, 2002. p.1143-1152.

IMMANUEL, C. D.; DOYLE III, F. J. Computationally efficient sollution of population balance models incorporating nucleation, growth and coagulation: application to emulsion polymerization. Chemical Engineering Science, v.58, 2003. p.3681-3698.

KONG, X. Z.; PICHOT, C.; GUILLOT, J. Kinects of emulsion copolymerization of vinyl acetate with butyl acrylate. European Polymer Journal, v.24, 1988. p.485-492.

LI, B.; BROOKS, B. W. Prediction of the average number of radicals per particle for emulsion polymerization. Journal of Polymer Science Part A: Polymer Chemistry, v.31(9), 1993. p.2397-2402.

MANDERS, B. G.; CHAMBARD, G.; KINGMA, W. J.; KLUMPERMAN, B.; HERK, A. M.; GERMAN, A. L. Estimation of activation parameters for the propagation rate 
constant of styrene. Journal of Polymer Science Part A: Polymer Chemistry, v.34, 1996. p.2473-2479.

MARINANGELO, G. Copolimerização em emulsão de estireno e acrilato de butila com alto teor de sólidos: Estudo experimental e modelagem matemática do processo em reator semicontínuo. Tese de Doutorado, Universidade de São Paulo, Escola Politécnica, Brasil: [s.n.], 2010.

MARINANGELO, G.; HIROTA, W. H.; GIUDICI, R. Semi-batch emulsion copolymerization of styrene and butyl acrylate for production of high solids contents latexes: Experiments and mathematical model. Chemical Engineering Science, v.66, 2011. p.5875-5890.

MCKENNA, T. F.; GRAILLAT, C.; GUILLOT, J. Contributions to defining the rate constants for the homo- and copolymerisation of butyl acrylate and vinyl acetate. Polymer Bulletin, v.34, 1995. p.361.

MEDOWS, E. S.; CROWLEY, T. J.; IMMANUEL, C. D.; DOYLE III, F. J. Nonisotermal modeling and sensitivity studies for batch and semibatch emulsion polymerization of styrene. Industrial and Engineering Chemistry Research, v.42, 2003. p.555-567.

ODIAN, G. Principles of Polymerization. 3a. ed. [S.I.]: Jonh Wiley \& Sons, 1991. Cap. 4.

OMI, S.; KUSHIBIKI, K.; NEGISHI, M.; ISO, M. Generalized computer modeling of semi-batch, n-component emulsion copolymerization and its applications. [S.I.]: Gijutsu, Zairyo, v. 3, 1985. p. 426.

PLESSIS, C.; ARZAMENDI, G.; LEIZA, J. R.; SCHOONBROOD, H. A. S.; CHARMOT, D.; ASUA, J. M. Kinect and Polymer microstructure of the seeded semibatch emulsion copolymerization of n-butyl acrylate and styrene. Macromolecules, v.34, 2001. p.5147-5157.

RAWLINGS, J. B.; RAY, W. R. The modelling of batch and continuous emulsion polymerization reactors: II. comparison with experimental data from continuous stirred tank reactor. Polymer Engineering Science, v.28, 1988. p.257-274. 
SALAZAR, A.; GUGLIOTTA, L. M.; VEGA, J. R.; MEIRA, G. R. Molecular Weight Control in a Starved Emulsion Polymerization of Styrene. Industrial Engineering and Chemical Research, v.37, 1998. p.3582-3591.

SALDÍVAR, E.; ARAUJO, O.; GIUDICI, R.; LÓPEZ-BARRÓN, C. Modeling and Experimental Studies of Emulsion Copolymerization Systems. II. Styrenics. Journal of Applied Polymer Science, v.79, 2001. p.2380-2397.

TAYLOR, D. R.; VAN BERKEL, K. Y.; ALGHAMDI, M. M.; RUSSELL, G. T. Termination rate coefficients for radical homopolymerization of methyl methacrylate and styrene at low conversion. Macromolecular Chemistry and Physics, v.211, 2010. p.563-579.

THICKETT, S. C.; GILBERT, R. G. Emulsion Polymerization: state of art in kinectics and mechanisms. Polymer, v.48, 2007. p.6965-6991.

VALE, H. M.; MCKENNA, T. F. Modeling particle size distribution in emulsion polymerization reactors. Progress in Polymer Science, v.30, 2005. p.1019-1048.

WANG, W.; HUTCHINSON, R. A. High Temperature semibatch free radical copolymerization of styrene and butyl Acrylate. Macromolecular Symposia, v.289, 2010. p.33-42.

ZEAITER, J.; ROMAGNOLI, J. A.; BARTON, G. W.; GOMES, V. G.; HAWKETT, B. S.; GILBERT, R. G. Operational of semi-batch emulsion polymerization reactors: modelling validation and effect of operation conditions. Chemical Engineering Science, v.57, 2002. p.2955-2969. 


\section{Apêndice A}

O cálculo de radicais na fase aquosa para o parâmetro $z=1$ e $z=2$ não segue 0 sistema de equações apresentadas no tópico 4.6. Portanto, os mesmos serão apresentados neste apêndice e fazem parte do modelo.

Caso 1:

$z=1$

$\left[\dot{R}_{T}\right]_{a q}=\sum_{n=1}^{j c r i t-1}\left[\dot{R}_{n}\right]_{a q}$

$\left[\dot{R}_{1}\right]_{a q}=\frac{2 \cdot f \cdot k_{d} \cdot[I]+\frac{k_{d e s}}{V_{R}} \cdot\left[\int_{r_{\text {muc }}}^{r_{\max }} \tilde{n}(r, t) \cdot F(r, t) \cdot d r\right] \cdot}{\sum_{j}^{A, B} k_{p j, a q} \cdot\left[M_{j}\right]_{a q}+\bar{k}_{t, a q} \cdot\left[\dot{R}_{T}\right]_{a q}+\left(\frac{k_{e} \cdot N_{A} \cdot \int_{r_{\text {muc }}}^{r_{\max }} F(r, t) \cdot d r+k_{\text {emic }} \cdot N_{\text {mic }}}{V_{R} \cdot N_{A}}\right)}$

$\left(n=2, \ldots, j_{\text {crit }}-1\right)$ :

$\left[\dot{R}_{n}\right]_{a q}=\frac{\left[\dot{R}_{n-1}\right]_{a q} \cdot \sum_{j}^{A, B} k_{p j, a q} \cdot\left[M_{j}\right]_{a q}}{\sum_{j}^{A, B} k_{p j, a q} \cdot\left[M_{j}\right]_{a q}+\bar{k}_{t, a q} \cdot\left[\dot{R}_{T}\right]_{a q}+\left(\frac{k_{e} \cdot N_{A} \cdot \int_{r_{\text {muc }}}^{r_{\max }} F(r, t) \cdot d r+k_{e m i c} \cdot N_{m i c}}{V_{R} \cdot N_{A}}\right)}$ 


$$
\begin{aligned}
& \beta=\frac{\sum_{j}^{A, B} k_{p j, a q} \cdot\left[M_{j}\right]_{a q}}{\sum_{j}^{A, B} k_{p j, a q} \cdot\left[M_{j}\right]_{a q}+k_{t, a q} \cdot\left[\dot{R}_{T}\right]_{a q}+\left(\frac{k_{e} \cdot N_{A} \cdot \int_{r_{n u c}}^{r_{\max }} F(r, t) \cdot d r+k_{e m i c} \cdot N_{m i c}}{V_{R} \cdot N_{A}}\right)} \\
& {\left[\dot{R}_{n}\right]_{a q}=\left[\dot{R}_{n-1}\right]_{a q} \cdot \beta} \\
& {\left[\dot{R}_{j_{c r i t}-1}\right]_{a q}=\left[\dot{R}_{1}\right]_{a q} \cdot \beta^{j_{c r i t}-2}} \\
& {\left[\dot{R}_{T}\right]_{a q}=\left[\dot{R}_{1}\right]_{a q} \cdot\left[1+\sum_{n=2}^{j_{c r i t-1}} \beta^{n-1}\right]} \\
& {\left[\dot{R}_{m}\right]_{a q}=\left[\dot{R}_{T}\right]_{a q}}
\end{aligned}
$$

Caso 2:

$z=2$

$$
\left[\dot{R}_{1}\right]_{a q}=\frac{2 \cdot f \cdot k_{d} \cdot[I]+\frac{k_{d e s}}{V_{R}} \cdot\left[\int_{r_{n u c}}^{r_{\max }} \tilde{n}(r, t) \cdot F(r, t) \cdot d r\right]}{\sum_{j}^{A, B} k_{p j, a q} \cdot\left[M_{j}\right]_{a q}+\bar{k}_{t, a q} \cdot\left[\dot{R}_{T}\right]_{a q}} .
$$$$
\left(n=2, \ldots, j_{\text {crit }}-1\right) \text { : }
$$$$
\left[\dot{R}_{n}\right]_{a q}=\frac{\left[\dot{R}_{n-1}\right]_{a q} \cdot \sum_{j}^{A, B} k_{p j, a q} \cdot\left[M_{j}\right]_{a q}}{\sum_{j}^{A, B} k_{p j, a q} \cdot\left[M_{j}\right]_{a q}+\bar{k}_{t, a q} \cdot\left[\dot{R}_{T}\right]_{a q}+\left(\frac{k_{e} \cdot N_{A} \cdot \int_{r_{n u c}}^{r_{\max }} F(r, t) \cdot d r+k_{e m i c} \cdot N_{m i c}}{V_{R} \cdot N_{A}}\right)}
$$ 
93

$$
\begin{aligned}
& \beta=\frac{\sum_{j}^{A, B} k_{p j, a q} \cdot\left[M_{j}\right]_{a q}}{\sum_{j}^{A, B} k_{p j, a q} \cdot\left[M_{j}\right]_{a q}+k_{t, a q} \cdot\left[\dot{R}_{T}\right]_{a q}+\left(\frac{k_{e} \cdot N_{A} \cdot \int_{r_{m u c}}^{r_{\max }} F(r, t) \cdot d r+k_{e m i c} \cdot N_{m i c}}{V_{R} \cdot N_{A}}\right)} \\
& {\left[\dot{R}_{n}\right]_{a q}=\left[\dot{R}_{n-1}\right]_{a q} \cdot \beta} \\
& {\left[\dot{R}_{j_{c r i t}-1}\right]_{a q}=\left[\dot{R}_{1}\right]_{a q} \cdot \beta^{j_{c r i t}-2}} \\
& {\left[\dot{R}_{T}\right]_{a q}=\left[\dot{R}_{1}\right]_{a q} \cdot\left[1+\sum_{n=2}^{j_{c r i t-1}} \beta^{n-1}\right]} \\
& {\left[\dot{R}_{m}\right]_{a q}=\left[\dot{R}_{T}\right]_{a q}}
\end{aligned}
$$




\section{Apêndice $B$}

Algoritmo para o Cálculo de Partição de Monômero

Estimar os valores de $V_{a q}, V_{d}$ e $V_{p}$ (OMI et al., 1985)

Calcular:

$$
\begin{aligned}
& V_{p}^{i}=\frac{V_{i}}{1+\frac{V_{a q}}{V_{p} \cdot K_{p}^{i}}+\frac{V_{d} \cdot K_{d}^{i}}{V_{p} \cdot K_{p}^{i}}} \\
& V_{a q}^{i}=\frac{V_{a q} \cdot V_{p}^{i}}{V_{p} \cdot K_{p}^{i}} \\
& V_{d}^{i}=\frac{V_{d} \cdot K_{d}^{i}}{V_{p} \cdot K_{p}^{i} \cdot V_{p}^{i}} \\
& V_{d}=\sum_{i=A, B} V_{d}^{i} \\
& V_{a q}=V_{H_{2} O}+\sum_{i=A, B} V_{a q}^{i} \\
& V_{p}=V_{p o l}+\sum_{i=A, B} V_{p}^{i}
\end{aligned}
$$

Realizar as iterações até que a diferença entre os valores de $V_{a q}, V_{d}$ e $V_{p}$ estimados e calculados seja menor que a tolerância especificada. 


\section{Apêndice C}

\section{Modelo Completo}

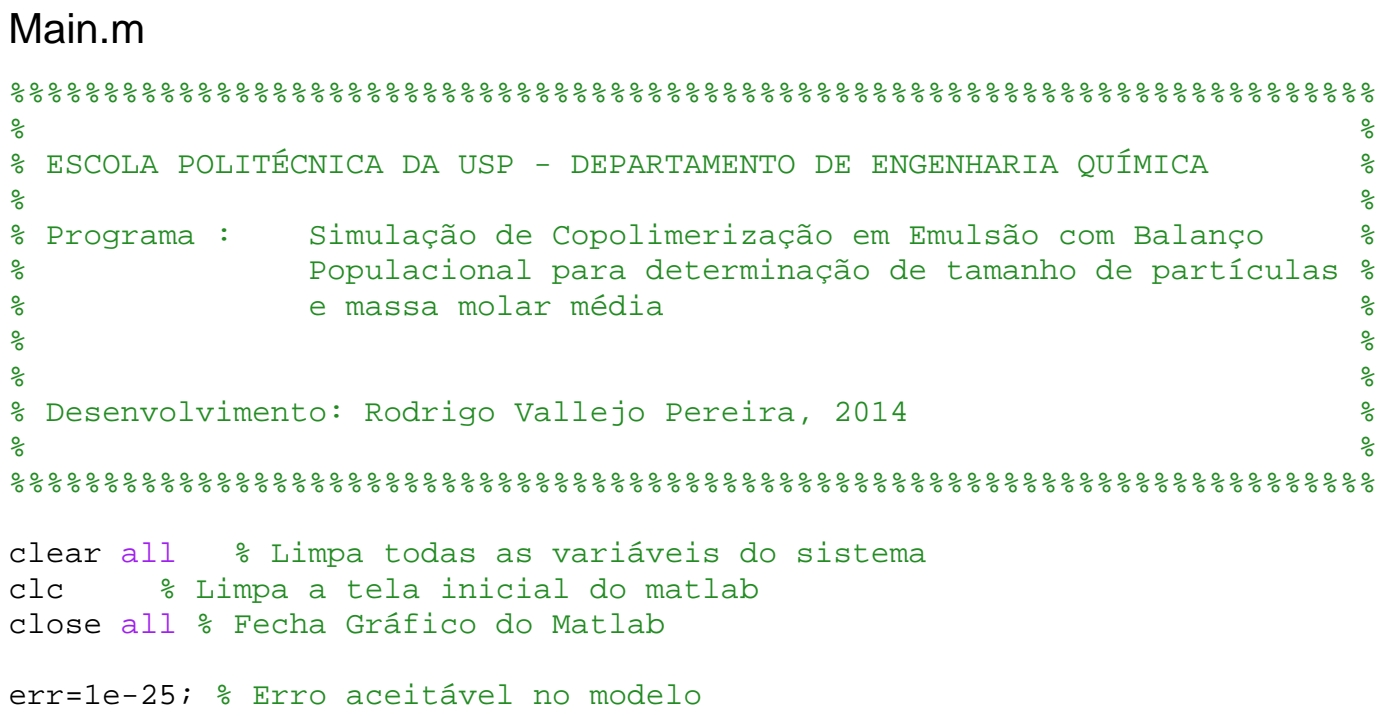




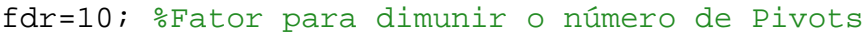
dr=fdr*1e-9; \% Passo para cálculo do Raio das Partículas (m) rmin=rmic $-(\mathrm{dr} . / 2)$; $\%$ Menor Raio da Partícula $(\mathrm{m}) \rightarrow r n u c=r m i c$ rmax=1000e-9; $\%$ Maior Raio da Partícula (m)

$r=r m i n: d r: r m a x ;$ ovetor em metros dos raio das partículas i_max=length $(r)-1$; $\frac{\circ}{0}$ Tamanho do vetor de pivots

n_medio=ones (i_max,1); oInicializa número m'dio de radicais por partículas Np_total_o=10;

$\mathrm{x}=\operatorname{zeros}\left(i \_m a x, 1\right)$; Inicializa o percentual de Particulas em cada Faixa $\mathrm{x}(1)=1$; $\frac{\circ}{0}$ stimativa inicial para $\mathrm{x}(1)$ - Total 100\%

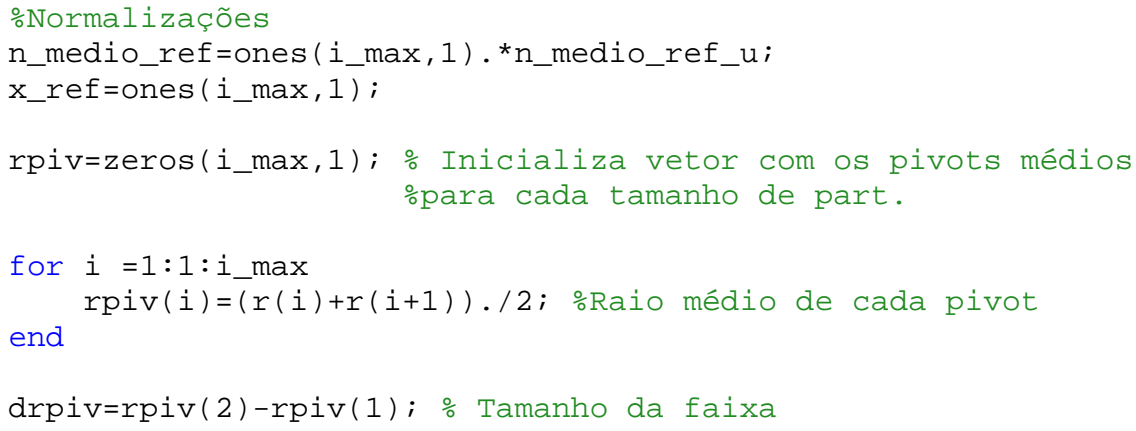




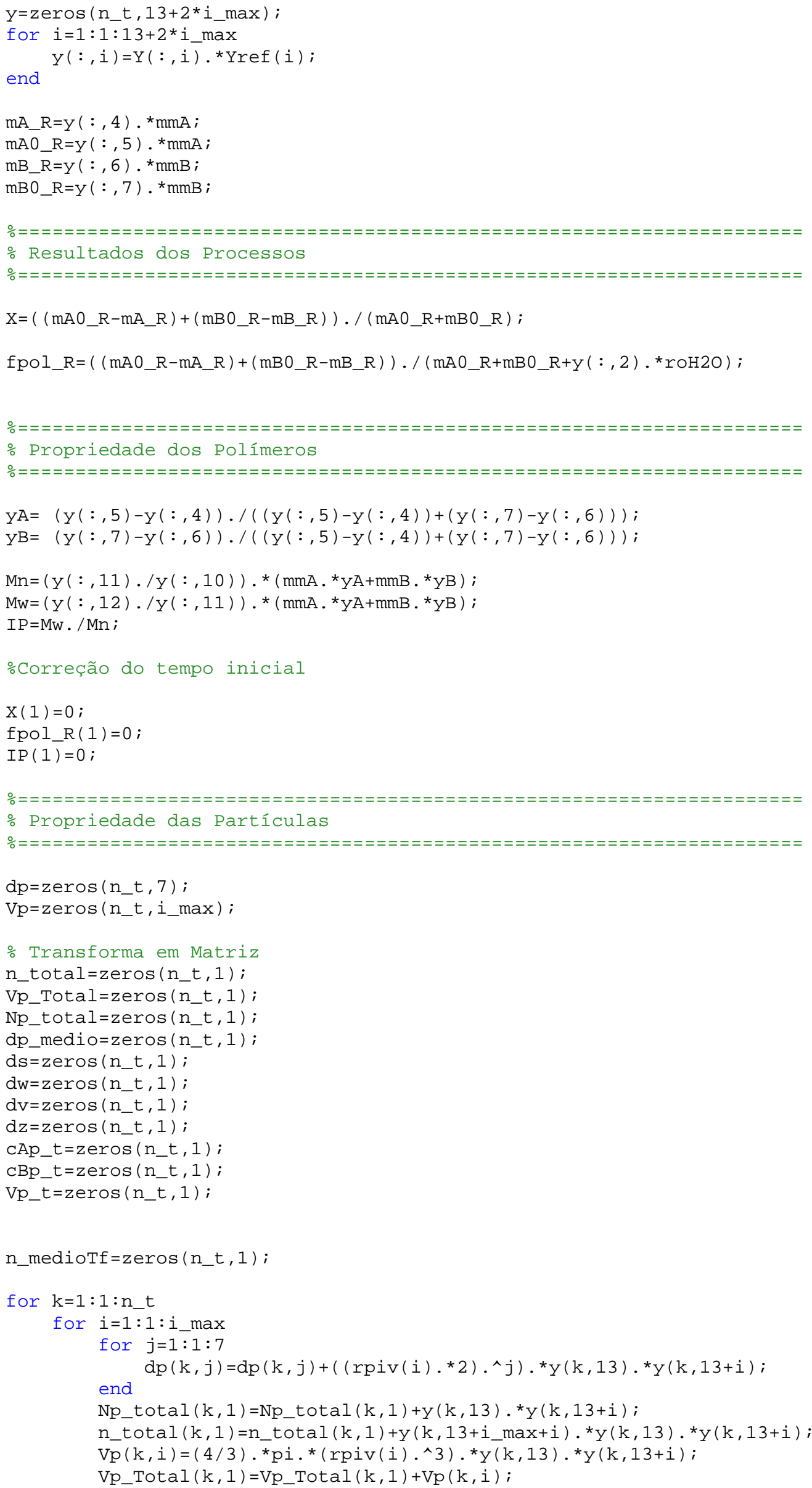


end

dp_medio $(k, 1)=d p(k, 1) . /$ Np_total $(k, 1) ;$ Numérico

$\mathrm{ds}(\mathrm{k}, 1)=\mathrm{dp}(\mathrm{k}, 3) \cdot / \mathrm{dp}(\mathrm{k}, 2) ;$ \% Sauter

$\mathrm{dw}(\mathrm{k}, 1)=\mathrm{dp}(\mathrm{k}, 4) \cdot / \mathrm{dp}(\mathrm{k}, 3) ;$ \% Massa

$\operatorname{dv}(k, 1)=(\operatorname{dp}(k, 3) . /$ Np_total $(k, 1)) \cdot \wedge(1 / 3) ;$ 은 Volume

$\mathrm{dz}(\mathrm{k}, 1)=(\mathrm{dp}(\mathrm{k}, 7) \cdot / \mathrm{dp}(\mathrm{k}, 6)) ; \quad \circ \mathrm{d} 76$

n_medioTf $(k, 1)=n \_t o t a l(k, 1)$. Np_total $(k, 1)$;

[ $C A p, c B p, C A a q, C B a q, V p \_R, V A p \_R, V B p \_R, V a q \_R, V A a q \_R, V B a q \_R, V A d r p \_R .$.

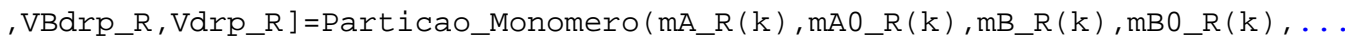
$\mathrm{y}(\mathrm{k}, 2)$, rоA, roPA, roB, roPB, $\mathrm{mmA}, \mathrm{mmB}, \mathrm{KpA}, \mathrm{KpB}, \mathrm{KdA}, \mathrm{KdB}, \operatorname{err}, \mathrm{t}(\mathrm{k}))$;

CAp_t $(\mathrm{k})=\mathrm{CAp}$;

$V p \_t(k)=V p \_R$;

end

oxporta para o Excel a Matriz Transposta

xlswrite (strcat ('resultados', ensaio),y');

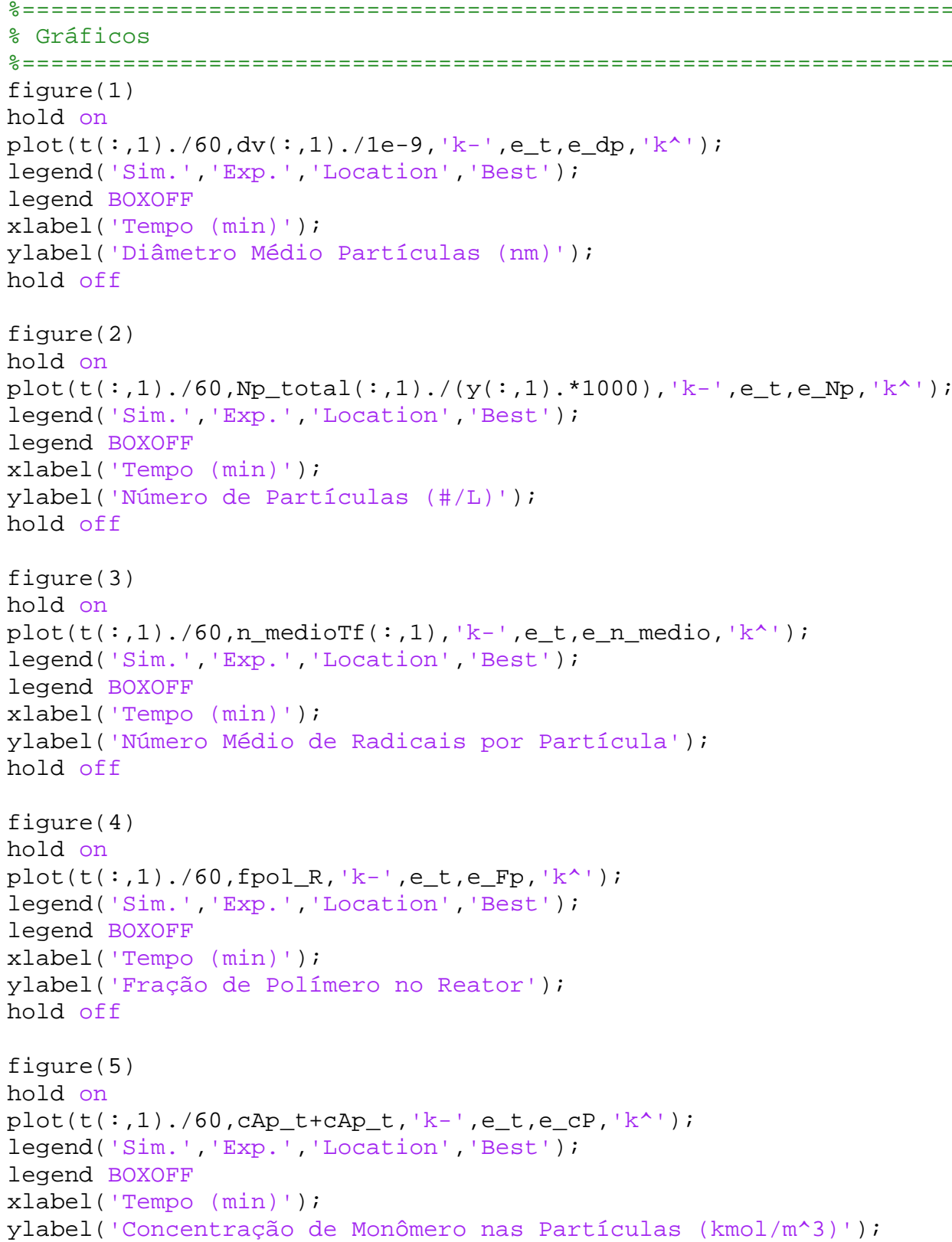


hold off

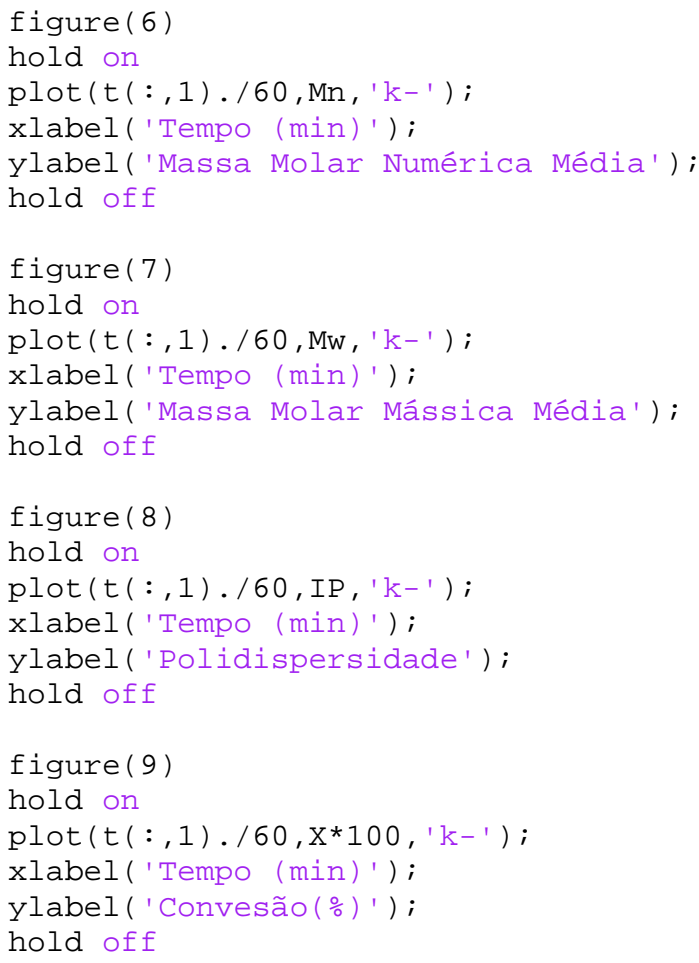




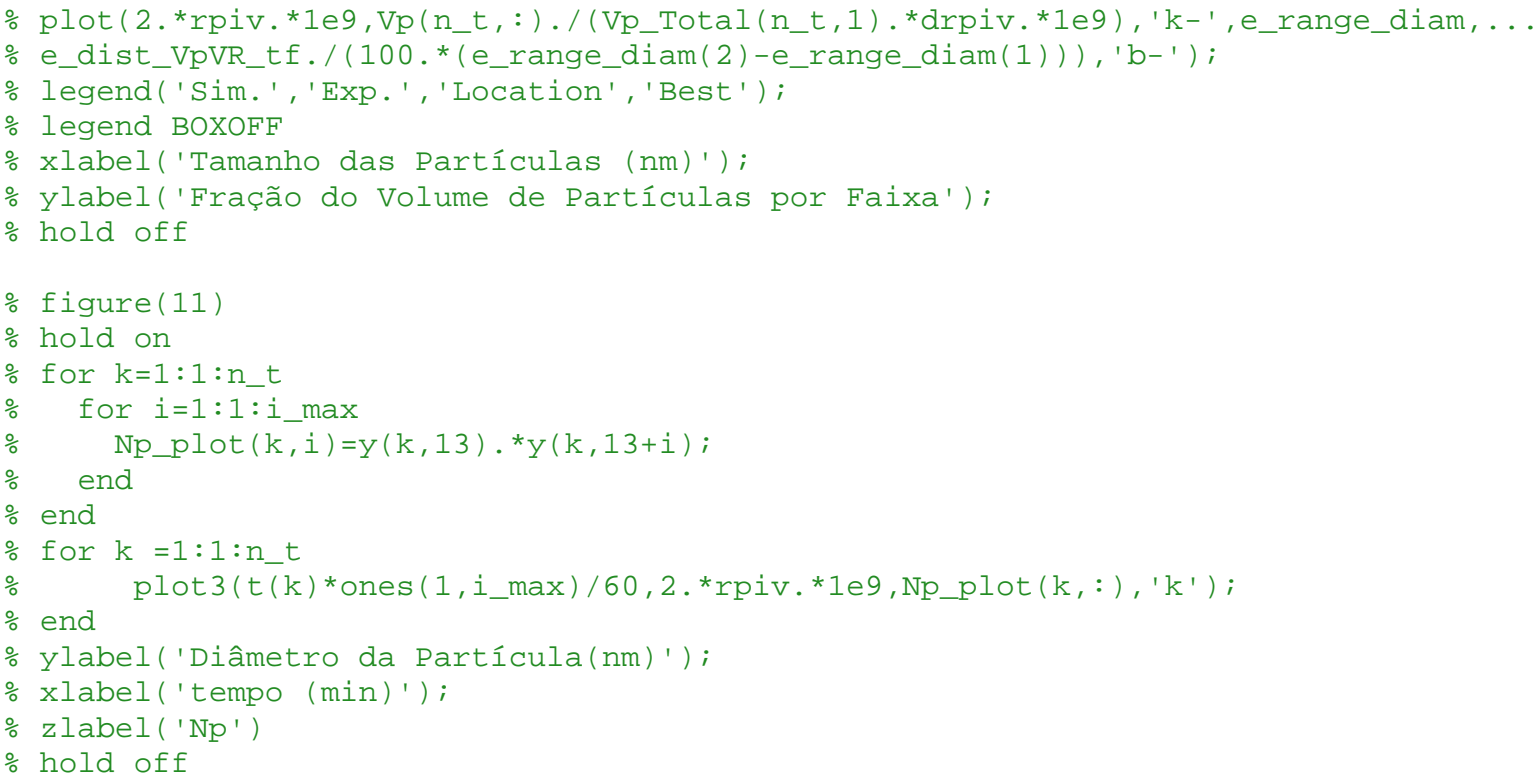




\section{Experimentos.m}

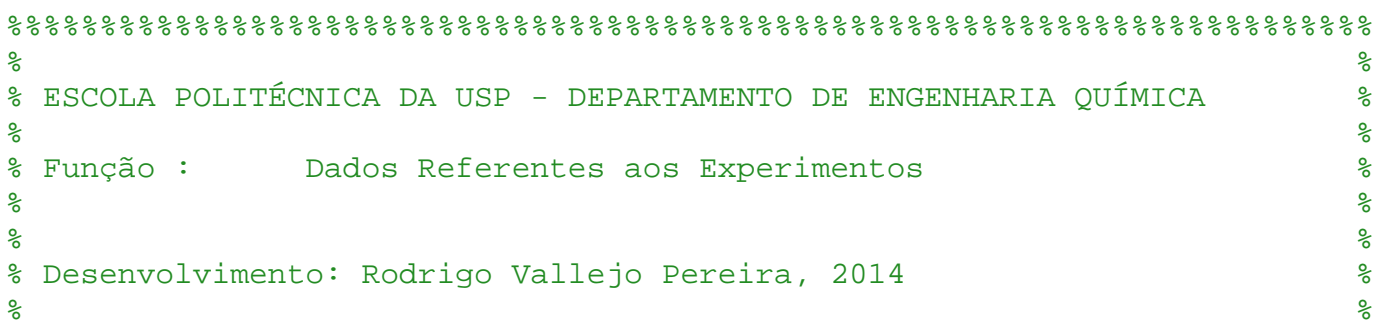

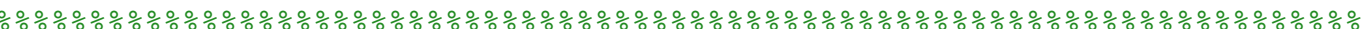

function [e_t, e_dp, e_Np, e_Fp, e_n_medio,e_cP, e_range_diam,e_dist_VpVR_180,... e_dist_Np_180, e_dist_VpVR_270,e_dist_Np_270, e_dist_VpVR_tf,...

e_dist_Np_tf]= Experimentos (ensaio)

switch lower (ensaio)

case 'a'\% Ensaio AS23 apresentado por Giovanni 2010

e_t $=\left[\begin{array}{llllllllllllllll}0 & 5 & 10 & 20 & 30 & 50 & 80 & 110 & 140 & 180 & 210 & 240 & 270 & 300 & 330 & 360\end{array}\right]$;

e_dp $=\left[\begin{array}{lllllllllllllll}0 & 0 & 198.6 & 174.3 & 193.1 & 228.1 & 292.3 & 376.3 & 465.7 & 576.3 & 594.9 & 586.6 & \ldots\end{array}\right.$ $634.5634 .6 \quad 635.8655 .3] ;$

e_Np $=\left[\begin{array}{llllllllllllll}0 & 0 & 0 & 1.13 e 15 & 2.24 e 15 & 3.30 e 15 & 3.72 e 15 & 3.19 e 15 & 2.80 e 15 & 2.70 e 15 & \ldots\end{array}\right.$

$3.16 e 153.85 e 15 \quad 3.18 e 153.26 e 15 \quad 3.26 e 15 \quad 2.93 e 15]$;

e_Fp $=\left[\begin{array}{lllllllllllllllll}0 & 0 & 0 & 0.013 & 0.030 & 0.059 & 0.107 & 0.159 & 0.224 & 0.337 & 0.435 & 0.509 & 0.531 \ldots\end{array}\right.$

$0.5490 .5520 .548]$;

e_n_medio $=\left[\begin{array}{lllllllllllllllllll}1 & 1 & 1 & 3.2 & 2.8 & 2.3 & 3.4 & 6.2 & 11.2 & 22.4 & 25.4 & 21.7 & 7.4 & 5.6 & 0.6 & 0\end{array}\right]$;

e_cP $=\left[\begin{array}{lllllllllllllllllll}0 & 0 & 7.23 & 6.86 & 6.55 & 6.25 & 5.78 & 5.31 & 4.70 & 3.65 & 2.60 & 1.81 & 1.57 & 1.38 & \ldots\end{array}\right.$

$1.351 .40] ;$ 을 Concetração de Monomeros nas Partículas

e_range_diam $=\left[\begin{array}{llllllllllllllll}0 & 100 & 100 & 200 & 200 & 300 & 300 & 400 & 400 & 500 & 500 & 600 & 600 & 700 & 700 & \ldots\end{array}\right.$ $800 \quad 800 \quad 900 \quad 900 \quad 1000 \quad 1000 \quad 1100 \quad 1100 \quad 1200 \quad 1200 \quad 1300 \quad 1300 \quad 1400 \quad 1400 \quad \ldots$

$150015001600]$; $\%$ nanometros (nm)

e_dist_VpVR_180=[ $\left[\begin{array}{llllllllllllllll}0.0 & 0.0 & 0.2 & 0.2 & 0.8 & 0.8 & 0.0 & 0.0 & 13.9 & 13.9 & 46.6 & 46.6 & \ldots\end{array}\right.$

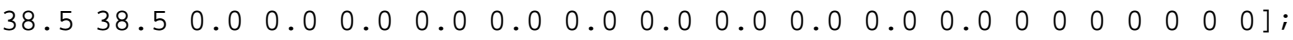
$\div \mathrm{V} / \mathrm{V}$ @180 $\mathrm{min}$

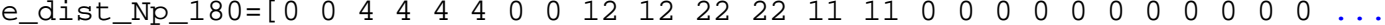

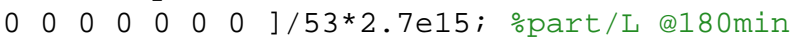

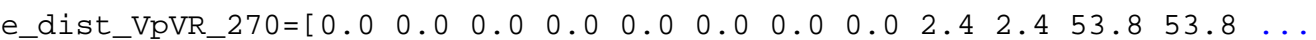

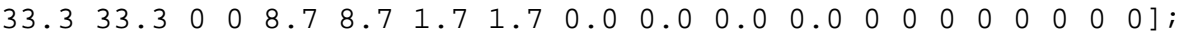
$\circ \mathrm{V} / \mathrm{V}$ @270 $\mathrm{min}$

e_dist_Np_270=[ $\begin{array}{lllllllllllllllllllllllll}0 & 0 & 0 & 0 & 1 & 1 & 0 & 0 & 13 & 13 & 160 & 160 & 60 & 60 & 0 & 0 & 7 & 7 & 1 & 1 & 0 & 0 & \ldots\end{array}$

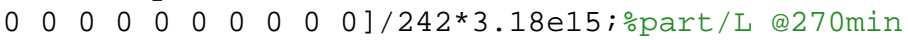

e_dist_VpVR_tf $=\left[\begin{array}{llllllllllllllllllllll}0.0 & 0.0 & 0.0 & 0.0 & 0.0 & 0.0 & 0.0 & 0.0 & 1.0 & 1.0 & 49.8 & 49.8 & \ldots\end{array}\right.$

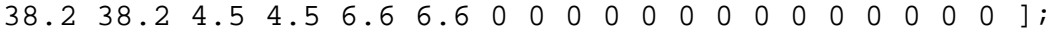

$\circ \mathrm{V} / \mathrm{V}$ atf $\mathrm{min}$

e_dist_Np_tf $=\left[\begin{array}{llllllllllllllllllllllllllll}0 & 0 & 0 & 0 & 0 & 0 & 0 & 0 & 1 & 1 & 28 & 28 & 13 & 13 & 1 & 1 & 1 & 1 & 0 & 0 & 0 & 0 & 0 & 0 & 0 & \ldots\end{array}\right.$

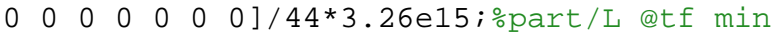

case 'b' Ensaio

e_t $=\left[\begin{array}{llllllllllllllll}0 & 5 & 10 & 20 & 30 & 50 & 80 & 110 & 140 & 180 & 210 & 240 & 270 & 300 & 330 & 360\end{array} ; ;\right.$ 


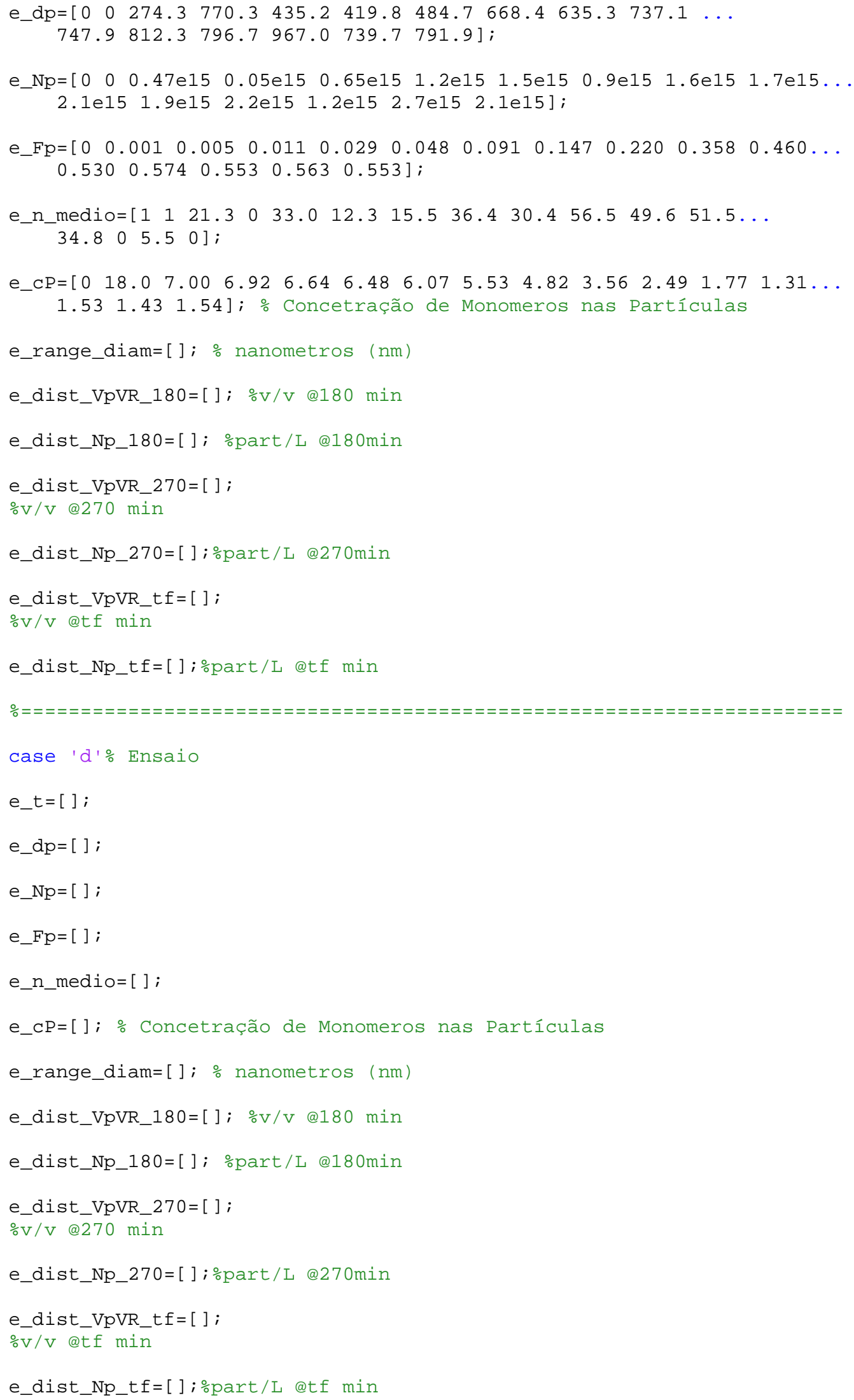




\section{Parametros.m}

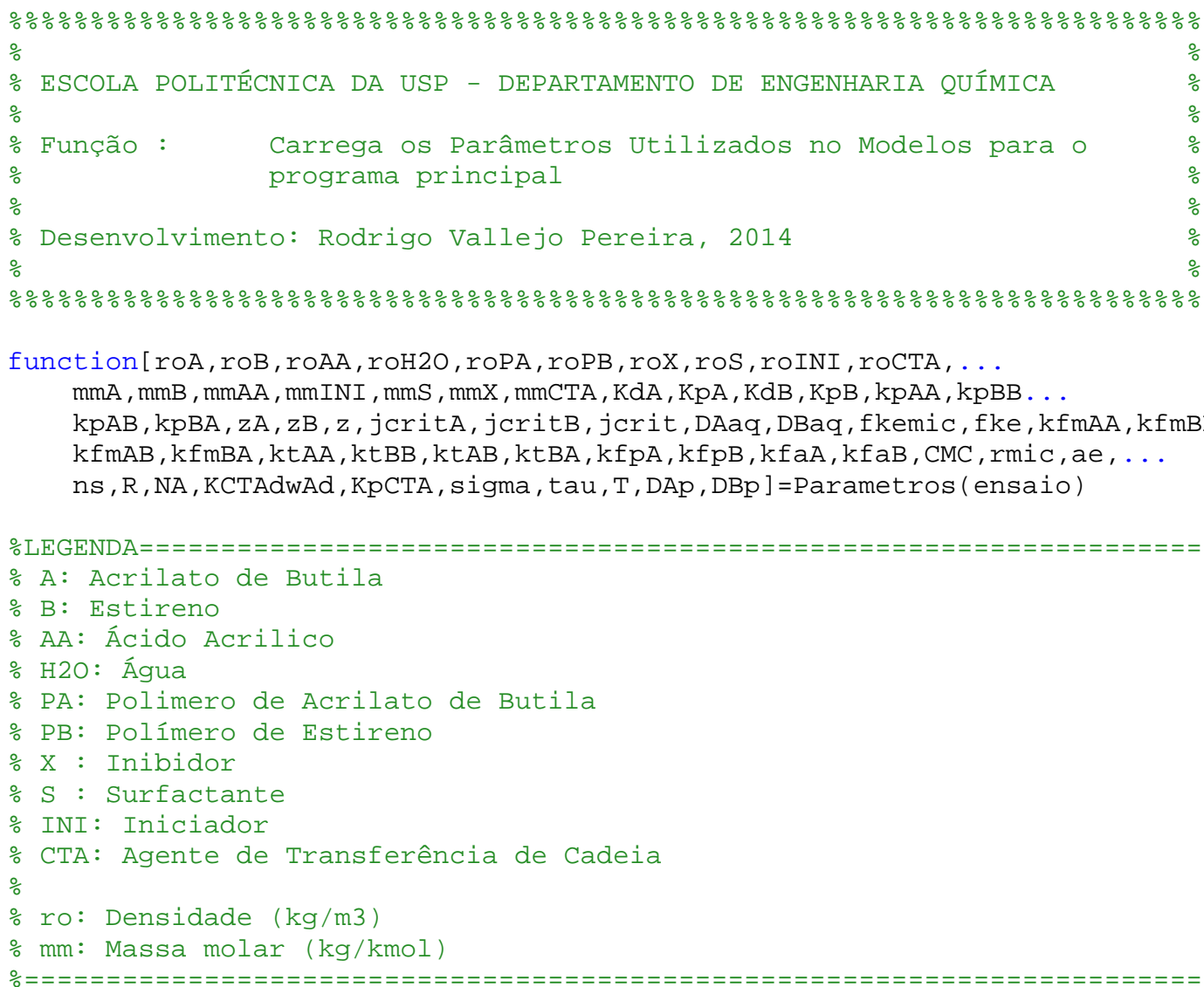

switch lower(ensaio)

case ' $a$ '

$\mathrm{T}=273.15+85$; $\frac{\circ}{\circ}$ Definição da Temperatura

$\mathrm{CMC}=2.6793 \mathrm{e}-4 ;$ ㅇmol/m3 - Medido em Laboratório Giovane

$a e=5.7 e-19$; OÁrea Especifica da partículas cobertas por uma molécula ode emulsificante

$\mathrm{fkemic}=0.25 e-8$

$\mathrm{fke}=0.15$;

case 'b'

$\mathrm{T}=273.15+85 ;$ 을 Definição da Temperatura

$\mathrm{CMC}=2.6793 \mathrm{e}-4 ; \circ \mathrm{kmol} / \mathrm{m} 3$ - Medido em Laboratório Giovane

ae=5.7e-19; 并ea Especifica da partículas cobertas por uma molécula ode emulsificante

fkemic $=0.25 e-8$

$\mathrm{fke}=0.15$;

case ' $d$ '

$\mathrm{T}=273.15+70 ;$ : Definição da Temperatura

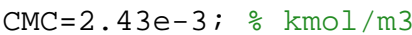

ae=1.97e-18; : Área Especifica da partículas cobertas por uma molécula \% de emulsificante

$f$ kemic $=(1.5 e-5)$

$\mathrm{fke}=0.02$;

end

$\circ \mathrm{GLOBAIS}=============$
$\mathrm{R}=8.314510 ; \% \mathrm{KJ} / \mathrm{kmol} \cdot \mathrm{K}$

$\mathrm{NA}=6.0221367 \mathrm{e} 26 ; \% \mathrm{moleculas} / \mathrm{kmol}$

$\because D E N S I D A D E(\mathrm{~kg} / \mathrm{m} 3)$

roA $=895.0$; $\div$ Acrilato de Butila - $\mathrm{T}=250 \mathrm{C}$ 


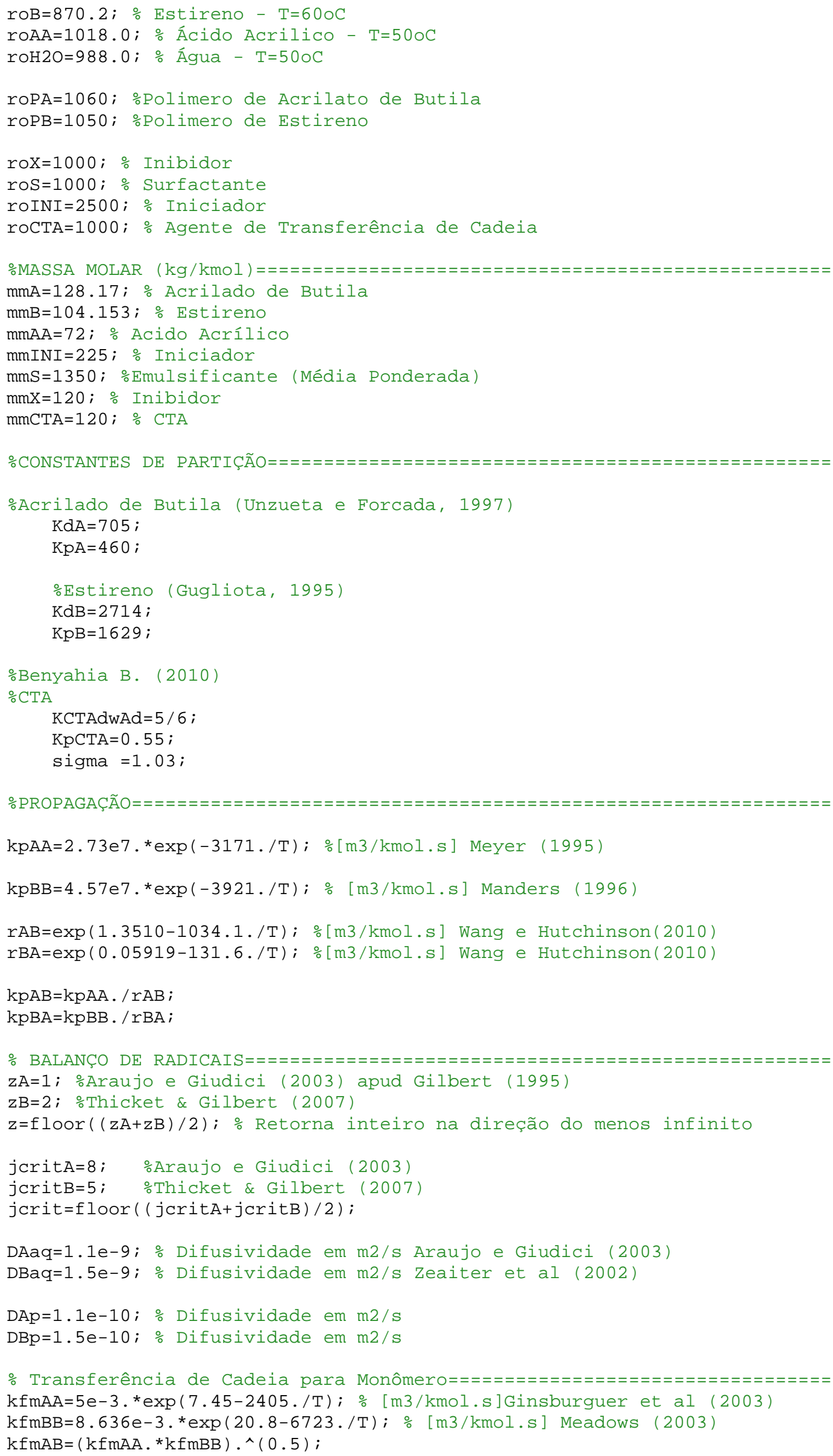




\section{$\mathrm{k} f \mathrm{mBA}=\mathrm{k}$ fmAB;}

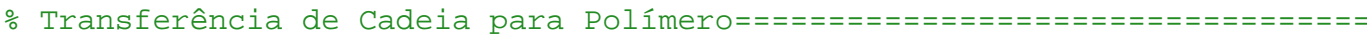

$\mathrm{kfpA}=2.48 \mathrm{e} 3 .{ }^{\star} \exp \left(-27700 /\left(8.3144 .{ }^{\star} \mathrm{T}\right)\right) ;$ ․ Hamzehlou et al (2014)

$\mathrm{kfpB}=(1.9 e-4) \cdot{ }^{\star} \mathrm{kpBB} ; \quad \frac{\circ}{0}$ amzehlou et al (2014)

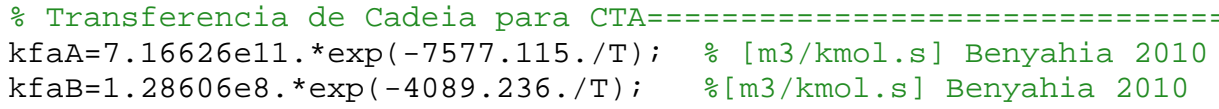

end 


\section{Cond_Contorno.m}

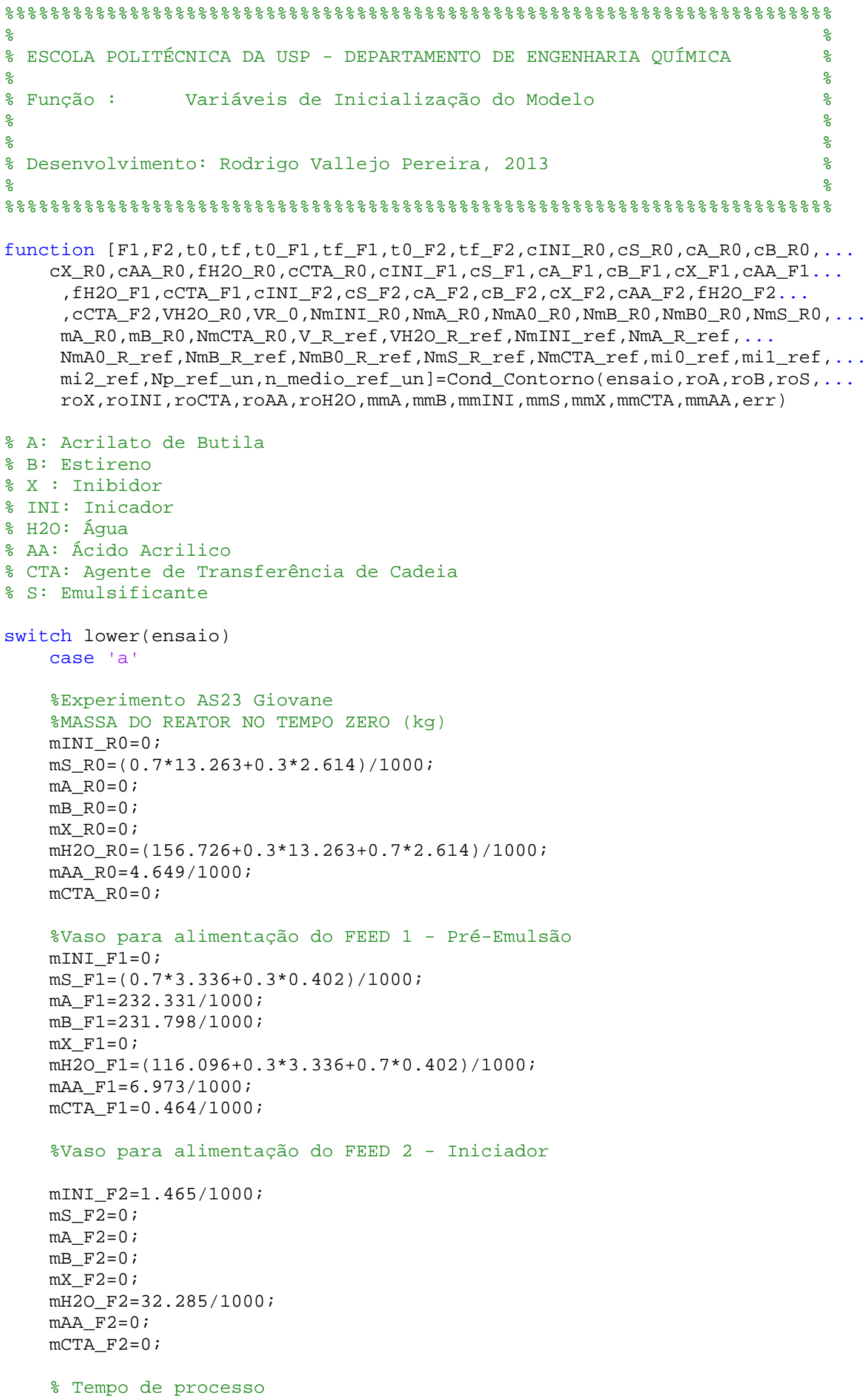


to=0; $\quad$ o Tempo Inicial da Reação em segundos

tf=21600; \%Tempo final da Reação em segundos

t0_F1=0; ำ Tempo Inicial da adição de pré-emulsão (F1)

tf_F1=10800; Tempo final da adição de pré-emulsão (F1)

t0_F2=0; Tempo Inicial da adição de Iniciador (F2)

tf_F2=12600; $\frac{0}{\circ}$ Tempo final da adição de Iniciador (F2)

case 'b'

-Experimento AS22 Giovane

\%MASSA DO REATOR NO TEMPO ZERO (kg)

mINI_RO=0; $\frac{\circ}{\circ}$ Massa do iniciador

$\mathrm{mS} \_\mathrm{RO}=(0.7 * 13.72+0.3 * 2.684) / 1000 ;$ Massa de Emusificante

$\mathrm{mA} R \mathrm{RO}=0$;

$\mathrm{mB} \_\mathrm{RO}=0$;

$\mathrm{mX} \_\mathrm{RO}=0$;

mH2O_RO $=(135.763+13.72 * 0.3+2.684 * 0.7) / 1000 ; \div$ Massa de Água

mAA_RO=0; $\%$ Massa de Ácido Acrílico

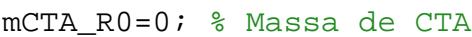

Vaso para alimentação do FEED 1 - Pré-Emulsão

mINI_F1=0;

mS_F $1=(0.7 * 4.4684+0.3 * 0.888) * 0.76982 / 1000$;

$\mathrm{mA} \_\mathrm{F} 1=311.8 * 0.76982 / 1000$;

mB_F1 $=312.0 * 0.76982 / 1000$;

$\mathrm{MX} \_\mathrm{F} 1=0$;

$\mathrm{mH} 2 \mathrm{O} \_\mathrm{F} 1=(156.076+0.3 * 4.468+0.7 * 0.888) * 0.76982 / 1000$;

mAA_F1 $1=0$;

MCTA_F $1=0.626 * 0.76982 / 1000$;

○Vaso para alimentação do FEED 2 - Iniciador

MINI_F $2=3.164 * 0.5 / 1000$;

$\mathrm{mS} \_\mathrm{F} 2=0$;

$\mathrm{mA} F 2=0$;

$\mathrm{MB} \_\mathrm{F} 2=0$;

$\mathrm{mX} F 2=0$;

$\mathrm{mH} 2 \mathrm{O} \_\mathrm{F} 2=64.34 * 0.5 / 1000$;

MAA_F2 $=0$;

$\mathrm{MCTA} F 2=0$

Tempo de processo

to=0; $\quad$ o Tempo Inicial da Reação em segundos

tf=21600; \%Tempo final da Reação em segundos

t0_F1=0; Tempo Inicial da adição de pré-emulsão (F1)

tf_F1=10800; \%Tempo final da adição de pré-emulsão (F1)

t0_F2=0; Tempo Inicial da adição de Iniciador (F2)

tf_F2=12600; $\circ$ Tempo final da adição de Iniciador (F2)

case 'd'

Experimento R3 - Benyahia

-MASSA DO REATOR NO TEMPO ZERO (kg)

mINI_R0=1/1000; $\frac{\circ}{0}$ Massa do iniciador

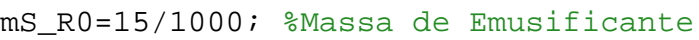

$\mathrm{mA} R \mathrm{R}=60 / 1000$;

$\mathrm{mB}$-RO $=60 / 1000$;

$\mathrm{mX} R \mathrm{RO}=0$;

mH2O_R0=570/1000; \% Massa de Água

mAA_RO=0; Massa de Ácido Acrílico

mCTA_RO=0.3/1000; assa de CTA

\%Vaso para alimentação do FEED 1 - Pré-Emulsão 


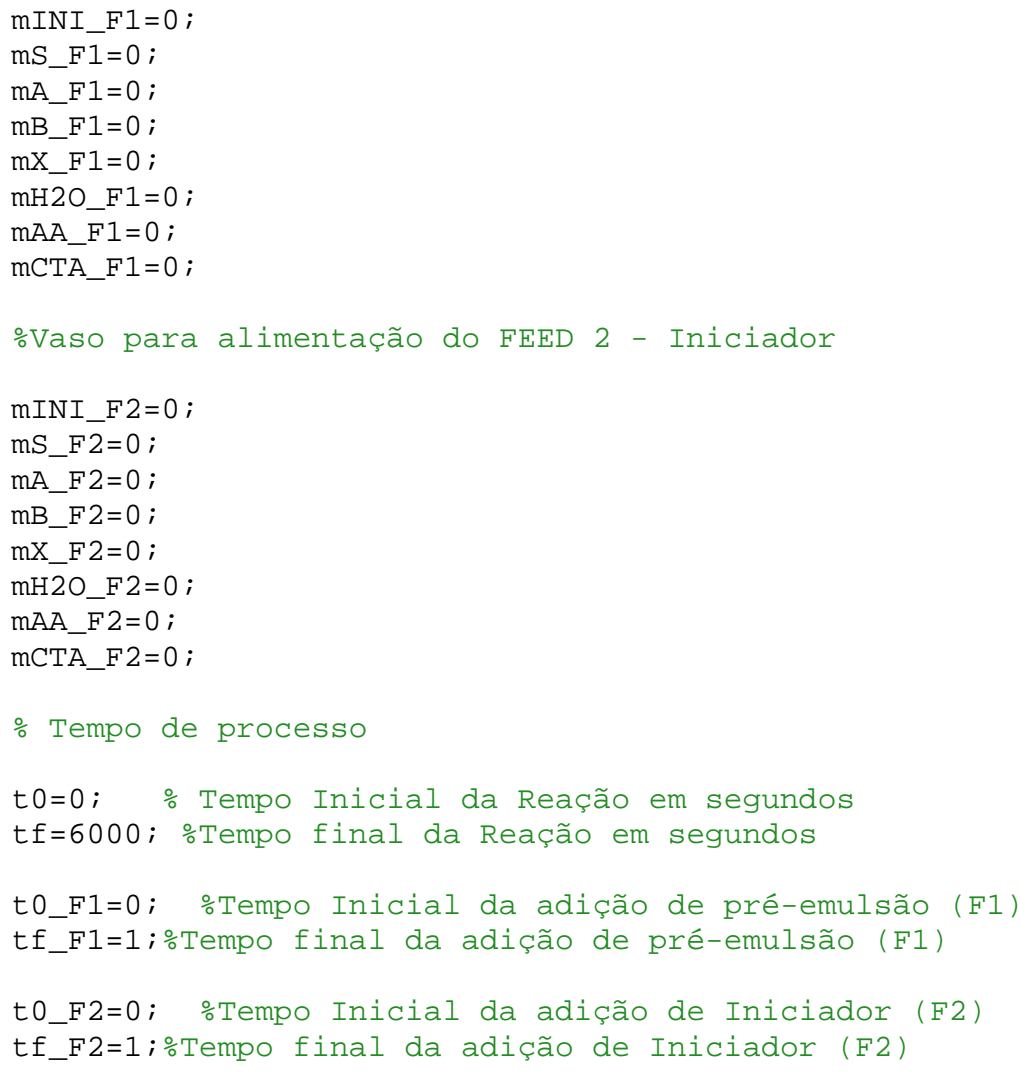


NmS_RO=VR_0.*CS_R0+err; Número de kmols de Emulsificante no Reator $t=0$; NmCTA_RO $=\overline{V R} \_0 .{ }^{*} \mathrm{C} C T A \_R 0+e r r ; \circ$ Número de kmols de CTA no Reator $t=0$;

\%Corrente F1

CINI_F1=(mINI_F1./mmINI)./VR_F1; Concentração de Iniciador em F1 t=0 CS_F1=(mS_F1./mmS)./VR_F1; Concentração de Emuslificante em F1 $t=0$ CA_F1=(mA_F1./mmA)./VR_F1; 0 Concentração de Monomero A em F1 $t=0$ CB_F1= (mB_F1./mmB)./VR_F1; 。 Concentração de Monomero B em F1 t=0 CX_F1= (mX_F1./mmX)./VR_F1; Concentração de Monomero C em F1 $t=0$ CAA_F1=(mAA_F1./mmAA)./VR_F1; 을 Concentração de Ácido Acrilico em F1 t=0 fH2O_F1=(mH2O_F1./rOH2O)./VR_F1; $\div$ fração de água em F1 t=0 CCTA_F1=(mCTA_F1./mmCTA)./VR_F1; \%Concentração de CTA em F1 t=0

응 Corrente F2

CINI_F2=(mINI_F2./mmINI)./VR_F2; Concentração de Iniciador em F2 t=0 CS_F2=(mS_F2./mmS)./VR_F2; Concentração de Emuslificante em F2 $t=0$ $\mathrm{CA} \_\mathrm{F} 2=\left(\mathrm{mA} \_F 2 . / \mathrm{mmA}\right) . / \mathrm{VR} \_F 2 ;$ o Concentração de Monomero A em F2 $t=0$ CB_F2 $=\left(\mathrm{mB} \_F 2 . / \mathrm{mmB}\right) . / \mathrm{VR} \_F 2 ;$ o Concentração de Monomero B em F2 $t=0$ CX_F2 $=\left(\mathrm{mX} \_F 2 . / \mathrm{mmX}\right)$./VR_F2; 0 Concentração de Monomero $C$ em F2 $t=0$ CAA_F2=(mAA_F2./mmAA) ./VR_F2; o Concentração de Ácido Acrilico em F2 t=0 fH2O_F2=(mH2O_F2./rOH2O)./VR_F2; 등 f̧ão de água em F2 t=0 CCTA_F2=(mCTA_F2./mmCTA)./VR_F2; oConcentração de CTA em F2 t=0

Referências para Normalização

$\circ======================$
V_R_ref $=$ VR_0+VR_F1+VR_F2;

$\mathrm{VH} 2 \mathrm{O} \_\mathrm{R} \_r e f=\left(\mathrm{mH} 2 \mathrm{O} \_\mathrm{RO} . / \mathrm{rOH} 2 \mathrm{O}\right)+\left(\mathrm{mH} 2 \mathrm{O} \_\mathrm{F} 1 . / \mathrm{rOH} 2 \mathrm{O}\right)+\left(\mathrm{mH} 2 \mathrm{O} \_\mathrm{F} 2 . / \mathrm{rOH} 2 \mathrm{O}\right)$;

NmINI_ref $=$ NmINI_R0+(mINI_F1./mmINI $)+($ mINI_F2./mmINI $)$;

NmA_R_ref $=\mathrm{NmA} \_\mathrm{R} 0+\left(\mathrm{mA} \_\mathrm{F} 1 . / \mathrm{mmA}\right)+\left(\mathrm{mA} \_\mathrm{F} 2 . / \mathrm{mmA}\right)$;

NmA0_R_ref $=$ NmA_R_ref;

NmB_R_ref $=$ NmB_R0 $+\left(\mathrm{mB} \_F 1 . / \mathrm{mmB}\right)+\left(\mathrm{mB} \_\mathrm{F} 2 . / \mathrm{mmB}\right)$;

NmBO_R_ref $=$ NmB_R_ref;

NmS_R_ref $=$ NmS_R0 $+\left(\mathrm{mS} \_F 1 . / \mathrm{mmS}\right)+\left(\mathrm{mS} \_\mathrm{F} 2 . / \mathrm{mmS}\right)$;

NmCTA_ref=NmCTA_R0+(mCTA_F1./mmCTA $)+\left(\mathrm{mCTA} \_F 2 . / \mathrm{mmCTA}\right)$;

mi0 ref $=1 e-7$;

mil_ref $=1 e-3$;

mi2_ref $=1$;

Np_ref_un $=1 \mathrm{e} 17$;

n_medio_ref_un $=1$;

end 


\section{Modelo.m}

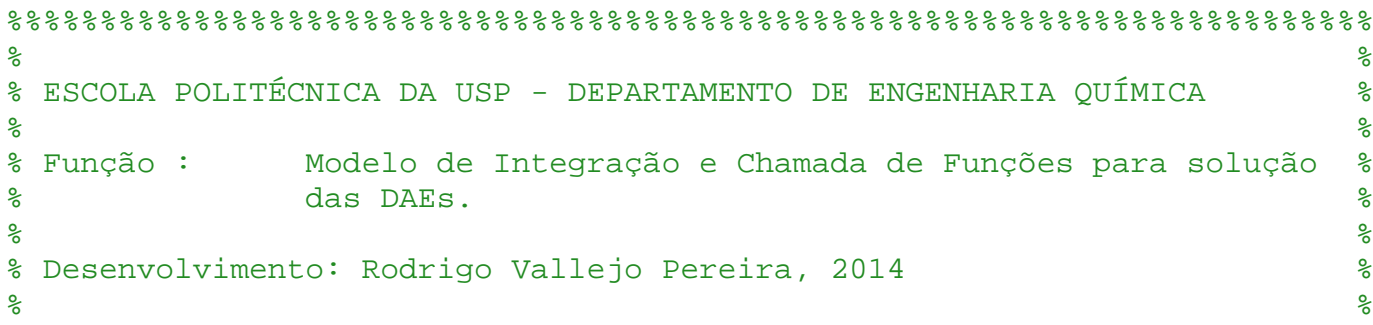

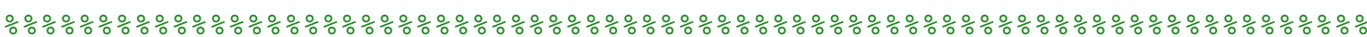

function $d y=$ Modelo $(t, y, T, r \circ A, r \circ P A, r o B, r \circ P B, m m A, m m B, K p A, K p B, K d A, K d B, \ldots$ $\mathrm{kpAA}, \mathrm{kpBB}, \mathrm{kpBA}, \mathrm{kpAB}, \mathrm{k} t A A, k t A B, k t B A, k t B B, k f m A A, k f m B B, k f m B A, k f m A B, \ldots$ $\mathrm{kfaA}, \mathrm{kfaB}, \mathrm{kfpA}, \mathrm{kfpB}, \mathrm{DAaq}, \mathrm{DBaq}$, fkemic, fke, rpiv, $\mathrm{x}, \mathrm{NA}, \mathrm{CMC}, \mathrm{ae}, \mathrm{ns}, \mathrm{rmic}, \ldots$ i_max, n_medio, $z, j c r i t, t 0 \_F 1, t 0 \_F 2, t f \_F 1, t f \_F 2, F 1, F 2, f H 2 O \_F 1, f H 2 O \_F 2, .$. CINI_F1, CINI_F2,CA_F1, CA_F2, CB_F1,CB_F2,CS_F1, CS_F2,CCTA_F1, CCTA_F2,... tau, dr, sigma, KpCTA, KCTAdwAd, err, ensaio, DAp, DBp)

oVariáveis Integradoras

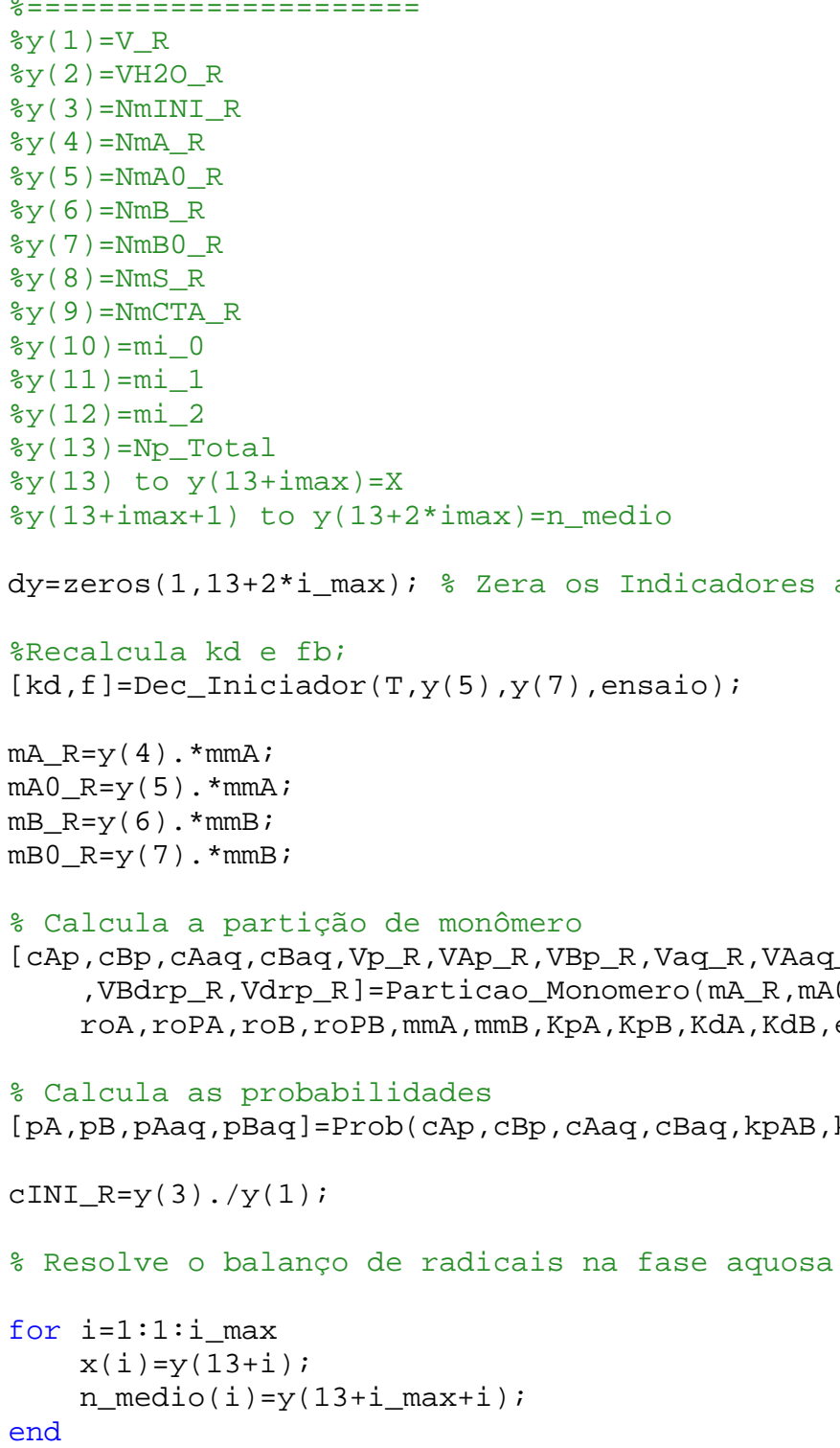

[cRlaq, cRTaq, Nmic, kemic, keNp, cRm, cRjcm1, n_total] =... Radicais_Fase_Aquosa (cAaq, cBaq, cAp, cBp, kpAA, kpBB, kpBA, kpAB, pAaq, ... $\mathrm{pBaq}, \mathrm{ktAA}, \mathrm{ktAB}, \mathrm{ktBA}, \mathrm{ktBB}, \mathrm{k} f \mathrm{mAA}, \mathrm{kfmBB}, \mathrm{k} f \mathrm{mBA}, \mathrm{kfmAB}, \mathrm{pA}, \mathrm{pB}, \mathrm{DAaq}, \mathrm{DBaq}, \ldots$ fkemic, fke, mmA, roA $, \mathrm{mmB}, \mathrm{roB}, \mathrm{rpiv}, \mathrm{x}, \mathrm{y}(13), \mathrm{NA}, \mathrm{y}(3), \mathrm{y}(8), \mathrm{CMC}, \mathrm{ae}, \mathrm{ns}, \ldots$ 
rmic, i_max, cINI_R, n_medio, f, kd, y (1) ,Vaq_R, z, jcrit, KpA, KpB, DAp, DBp, err) ;

oCalcula Balanço Populacional Número de Particulas

[Dx, dy (13) ] =Balanco_Pop (kpAA, kpBA, kpAB, kpBB, pAaq, pBaq, kemic, cRm, Nmic, cAaq. . . , $c B a q, N A, c R j c m 1, d r, n \_m e d i o, r p i v, p A, p B, c A p, m m A, r o P A, c B p, m m B, r o P B, \ldots$

i_max, Vaq_R, $x, y(13)$ );

oCalcula o Efito Gel

$[g]=$ Efeito_Gel (mmA, cAp, roA, roPA, mmB, cBp, roB, roPB, y (5), y (4), y (7), y (6), ... $\left.V p \_R, t, T\right)$;

oCalcula Balanço Populacional Número de Radicais por particula

[Dnmedio] =Balanco_Radicais (i_max, rpiv, mmA, $C A p, r o A, m m B, c B p, r o B, f k e, \ldots$

$\mathrm{NA}, \mathrm{DAaq}, \mathrm{DBaq}, \mathrm{pAaq}, \mathrm{pBaq}, \mathrm{pA}, \mathrm{pB}, \mathrm{cRm}, \mathrm{cRTaq}, \mathrm{kfmAA}, \mathrm{kfmBA}, \mathrm{kfmAB}, \mathrm{kfmBB}, \mathrm{n} \_m e d i o, \mathrm{kpA}, \ldots$ $\mathrm{kpAB}, \mathrm{kpBA}, \mathrm{kpBB}, \mathrm{ktAA}, \mathrm{ktBA}, \mathrm{ktAB}, \mathrm{ktBB}, \mathrm{g}, \mathrm{y}(1), \mathrm{cAaq}, \mathrm{cBaq}, \mathrm{y}(13), \mathrm{KpA}, \mathrm{KpB}, \ldots$ $\mathrm{DAp}, \mathrm{DBP}, \mathrm{x})$;

Balanços de Massa

[dy (1), dy (2) , dy (3), dy (4), dy (5), dy (6), dy (7), dy (8) , dy (9) , NmCTAp] ...

=Bal_Massa (kpAA, kpAB, kpBA, kpBB, pA, pB, pAaq, pBaq, cAp, cBp, cAaq, cBaq, ... CRTaq, NA, t , t0_F1, t0_F2, tf_F1, tf_F2, F1, F2, rOPA, rOA, $\mathrm{mmA}, \mathrm{rOPB}, \mathrm{rOB}, \mathrm{mmB}, \ldots$ fH2O_F1, fH2O_F2, $\mathrm{kd}, \mathrm{y}(3), \mathrm{CINI} F 1$, CINI_F2, CA_F1, CA_F2, CB_F1, CB_F2,... CS_F1, CS_F2, n_total, kfaA, $\mathrm{kfaB}, \mathrm{y}(9)$, CCTA_F1, CCTA_F2,Vaq_R, Vp_R, ... Vdrp_R, sigma, KpCTA, KCTAdwAd, kfmAA, kfmBB, kfmAB, kfmBA) ;

$\mathrm{CCTAp}=\mathrm{NmCTAp} . / \mathrm{Vp} \_\mathrm{R}$;

$[\mathrm{dy}(10), \mathrm{dy}(11), \mathrm{dy}(12)]=\ldots$

Balanco_Momentos (kpAA, kpAB, kpBA, kpBB, ktAA, ktAB, ktBA, ktBB, tau, ...

$\mathrm{kfmAA}, \mathrm{k} f \mathrm{mBB}, \mathrm{k} f \mathrm{mAB}, \mathrm{kfmBA}, \mathrm{kfpA}, \mathrm{kfpB}, \mathrm{kfaA}, \mathrm{kfaB}, \mathrm{pA}, \mathrm{pB}, \mathrm{NA}, \mathrm{Vp} \_\mathrm{R}, \mathrm{cAp}, \mathrm{cBp}, \ldots$ cCTAp, y (11), y(12), n_total,g);

for $i=1: 1: i \_\max$

$\operatorname{dy}(13+i)=\mathrm{Dx}(i)$;

dy $\left(13+i \_m a x+i\right)=$ Dnmedio $(i)$;

end

end 


\section{ModeloN.m}

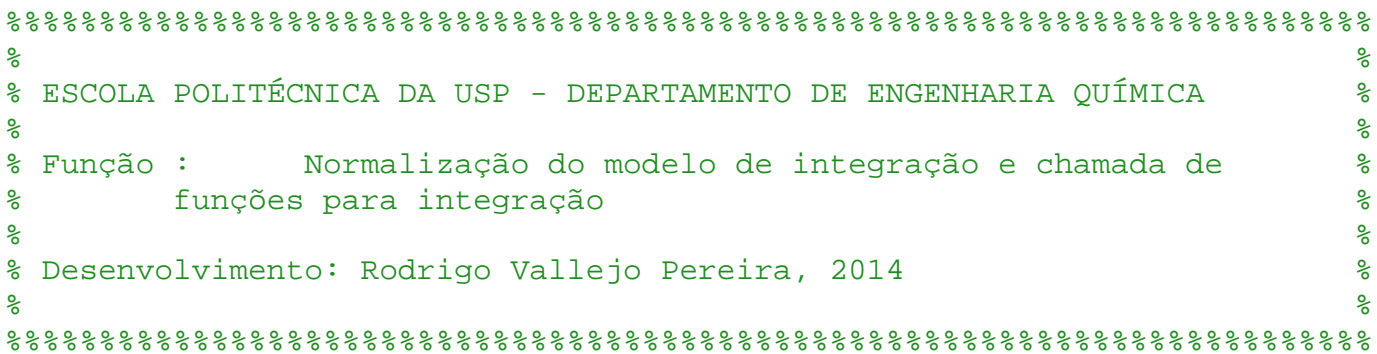

function $d Y=M o d e l \circ N(t, Y, T, r \circ A, r \circ P A, r o B, r \circ P B, m m A, m m B, K p A, K p B, K d A, K d B, \ldots$ $\mathrm{kpAA}, \mathrm{kpBB}, \mathrm{kpBA}, \mathrm{kpAB}, \mathrm{ktAA}, \mathrm{ktAB}, \mathrm{ktBA}, \mathrm{k} t \mathrm{BB}, \mathrm{kfmAA}, \mathrm{kfmBB}, \mathrm{kfmBA}, \mathrm{kfmAB}, \ldots$ $\mathrm{kfaA}, \mathrm{kfaB}, \mathrm{kfpA}, \mathrm{kfpB}, \mathrm{DAaq}, \mathrm{DBaq}$, fkemic, fke, rpiv, x, NA, CMC, ae, ns, rmic, i_max, ... n_medio,z,jcrit, t0_F1,t0_F2,tf_F1,tf_F2,F1,F2,fH2O_F1, fH2O_F2,... CINI_F1, CINI_F2, CA_F1, CA_F2, CB_F1, CB_F2, CS_F1, CS_F2, CCTA_F1, CCTA_F2,... tau, dr, sigma, KpCTA, KCTAdwAd, Yref, err, ensaio, DAp, DBp)

$y=\operatorname{zeros}\left(13+2 * i \_\max , 1\right)$;

for $i=1: 1: 13+2 * i \_\max$

end $\mathrm{Y}(\mathrm{i})=\mathrm{Y}(\mathrm{i}) . * \mathrm{Yref}(\mathrm{i})$;

$d y=$ Modelo $(t, y, T, r \circ A, r \circ P A, r \circ B, r \circ P B, m m A, m m B, K p A, K p B, K d A, K d B, \ldots$. $k p A A, k p B B, k p B A, k p A B, k t A A, k t A B, k t B A, k t B B, k f m A A, k f m B B, k f m B A, k f m A B, \ldots$ $\mathrm{kfaA}, \mathrm{kfaB}, \mathrm{kfpA}, \mathrm{kfpB}, \mathrm{DAaq}, \mathrm{DBaq}, \mathrm{fkemic}, \mathrm{fke}, \mathrm{rpiv}, \mathrm{x}, \mathrm{NA}, \mathrm{CMC}, \mathrm{ae}, \mathrm{ns}, \mathrm{rmic}, \ldots$ i_max, n_medio, $z$, jcrit, t0_F1, t0_F2, tf_F1, tf_F2,F1,F2, fH2O_F1, fH2O_F2,... CINI_F1, CINI_F2,CA_F1, CA_F2, CB_F1,CB_F2,CS_F1, CS_F2,CCTA_F1, CCTA_F2,... tau, dr, sigma, KpCTA, KCTAdwAd, err, ensaio, DAp, DBp) ;

$d Y=\operatorname{zeros}\left(13+2 * i \_m a x, 1\right)$;

for $i=1: 1: 13+2 * i \_\max$ end $d Y(i)=d y(i) . /$ Yref (i) :

end 


\section{Radicais_fase_aquosa.m}

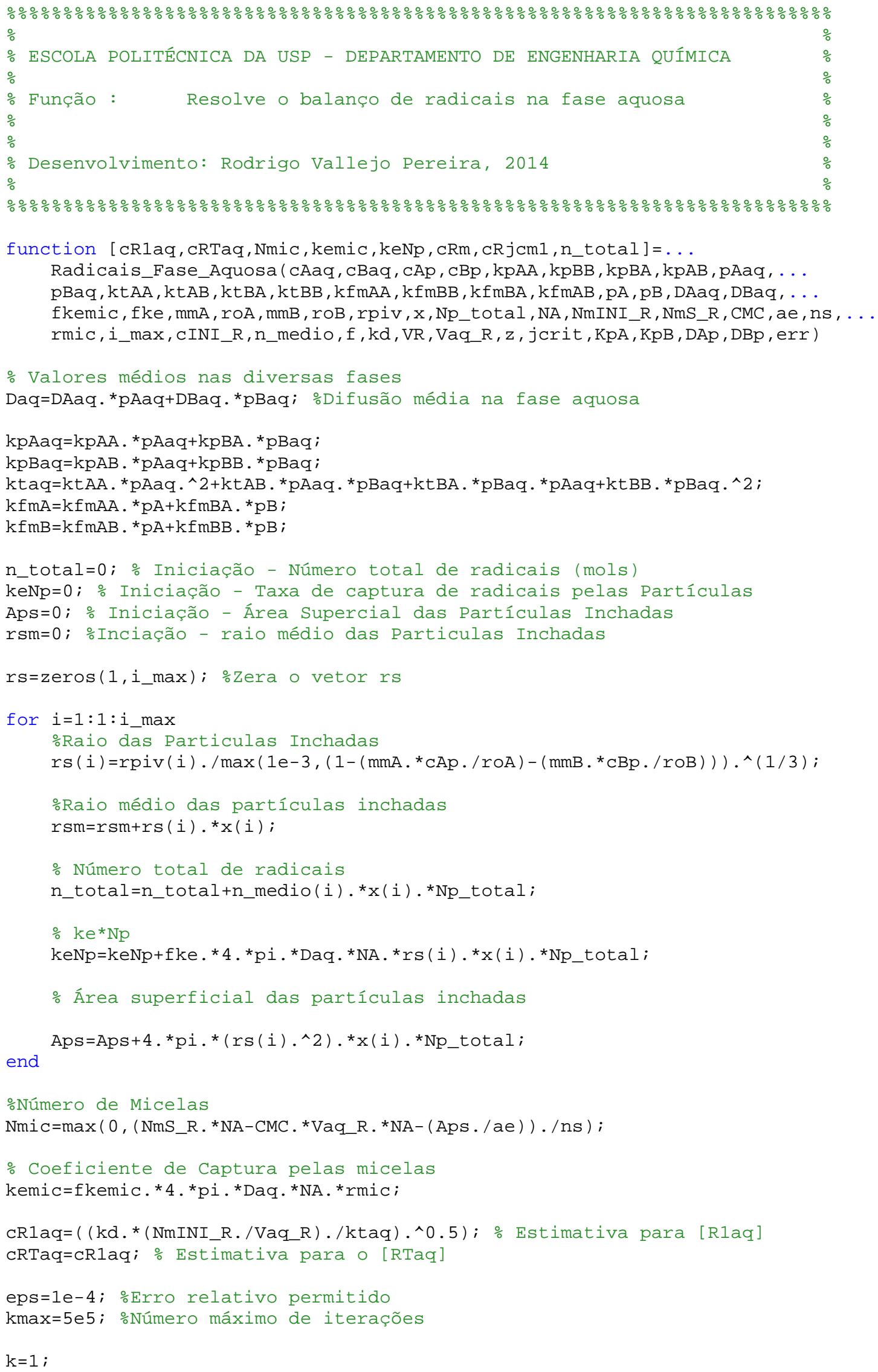




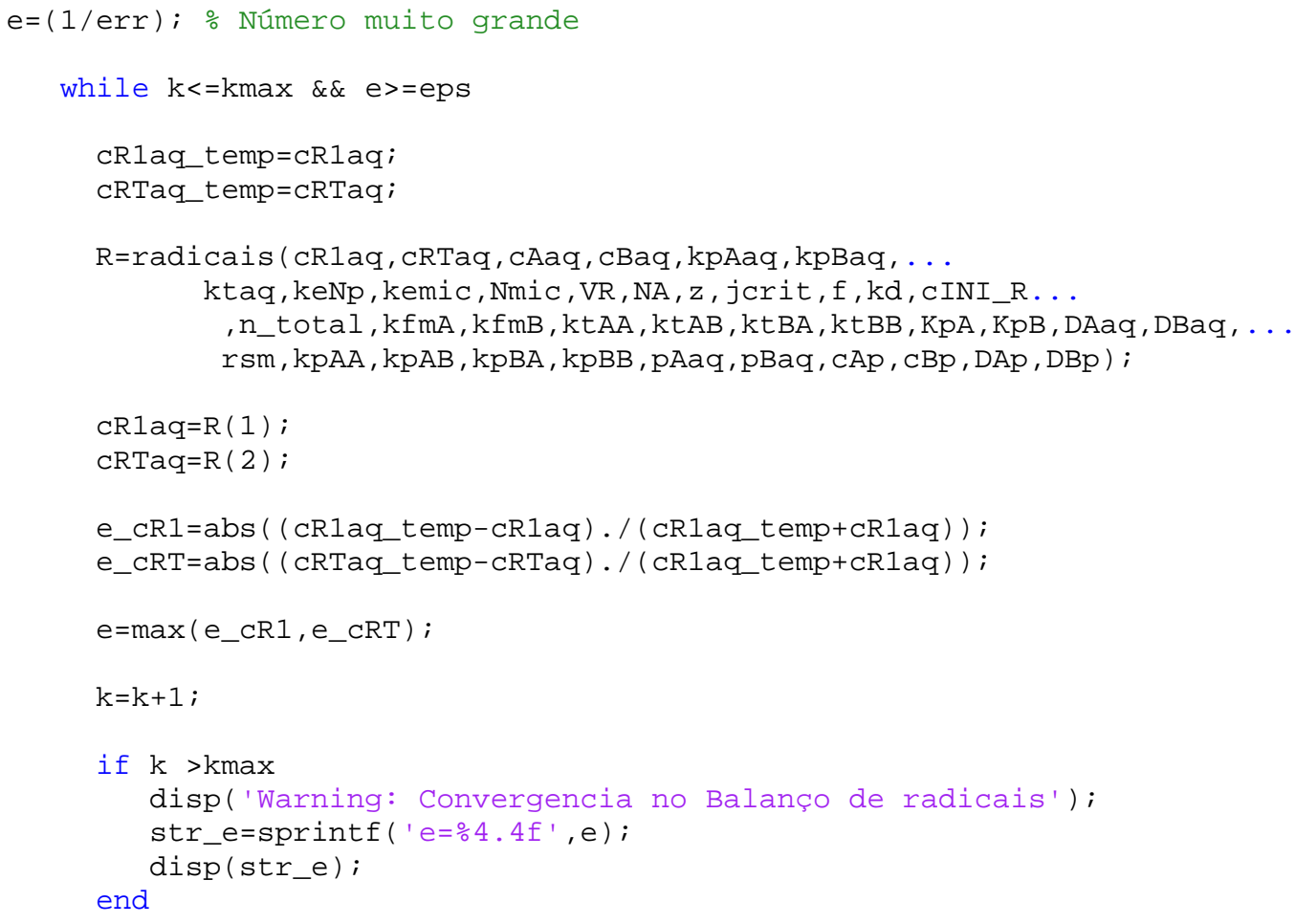




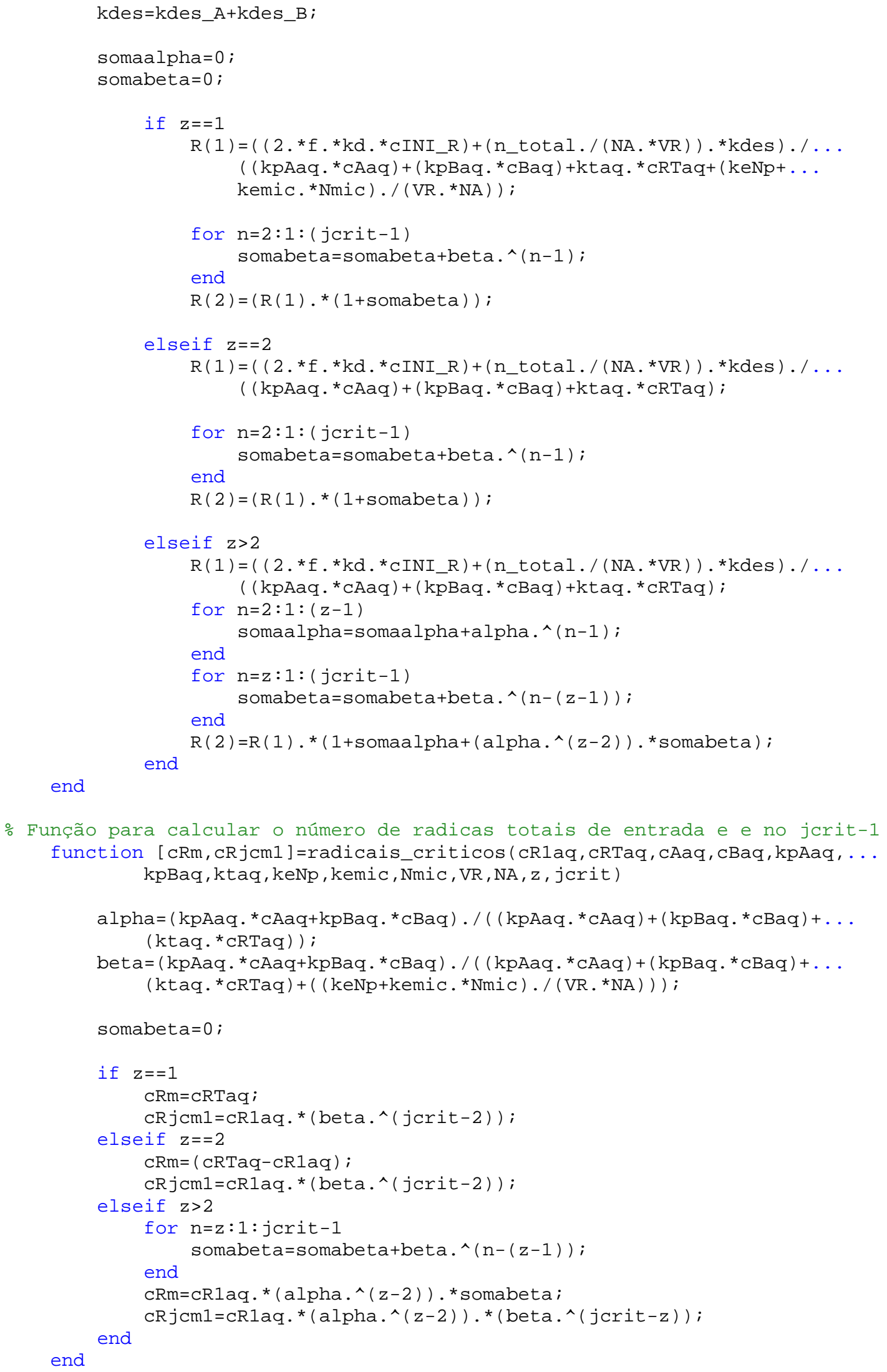




\section{Prob.m}

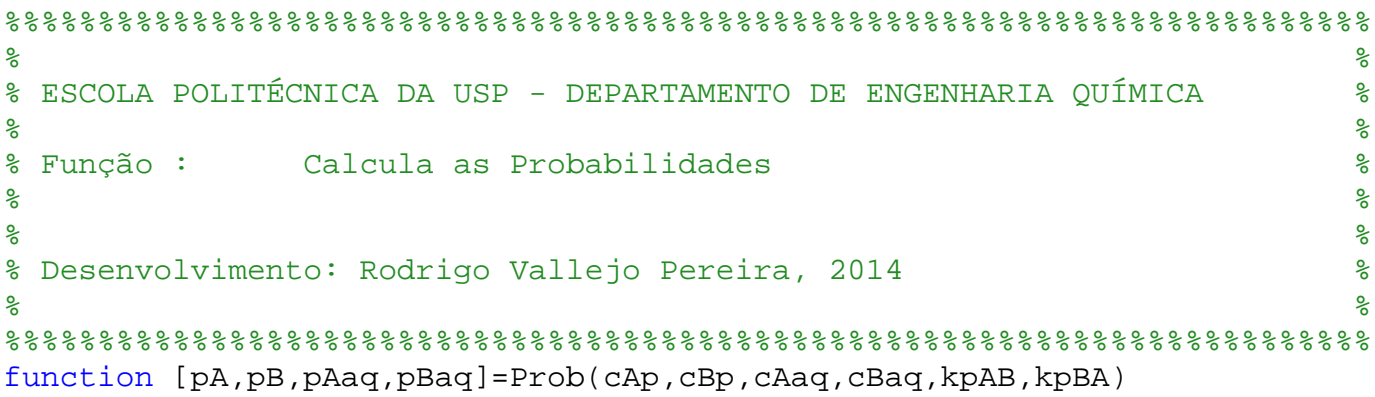

end 


\section{Particao_Monomeros.m}

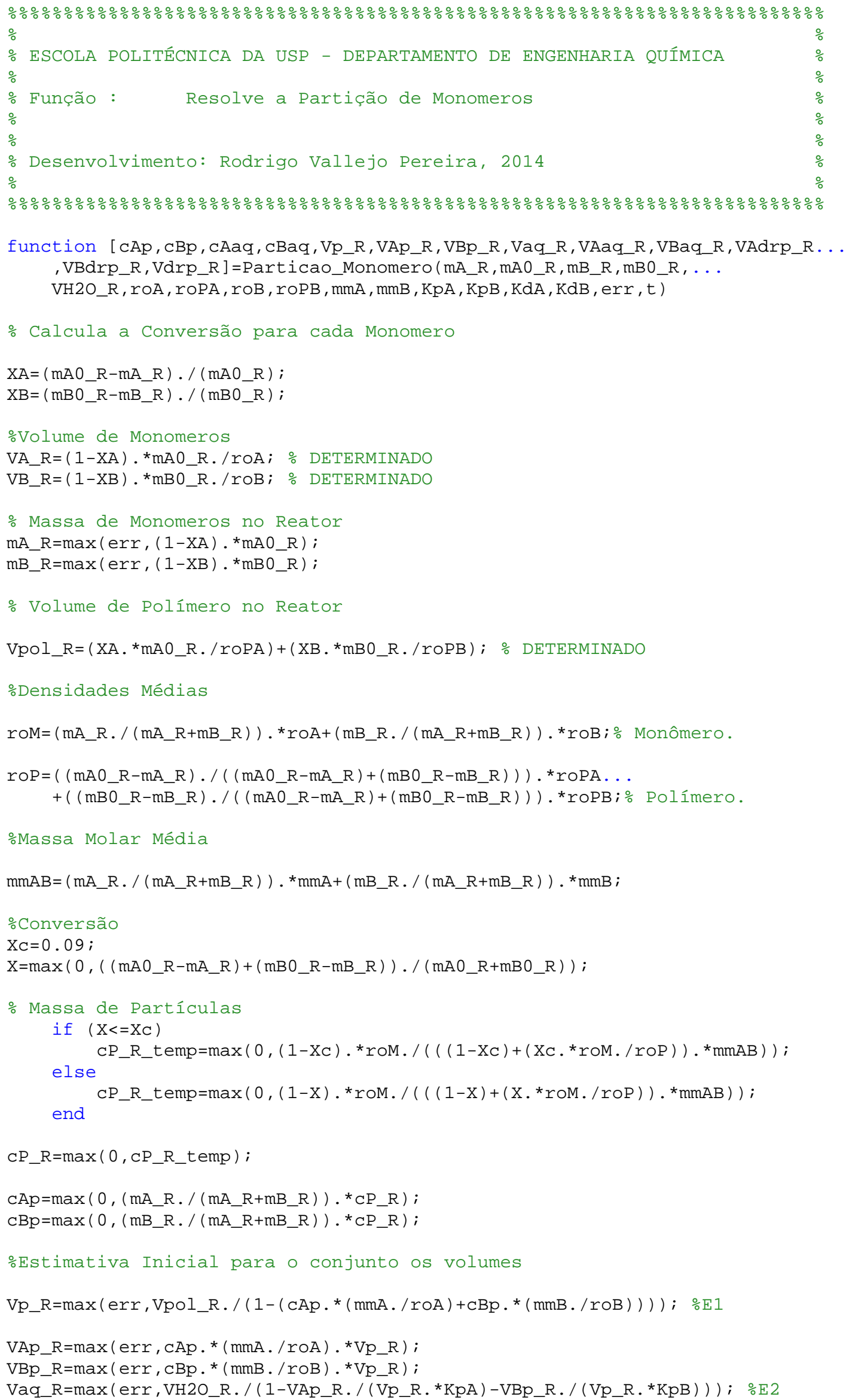




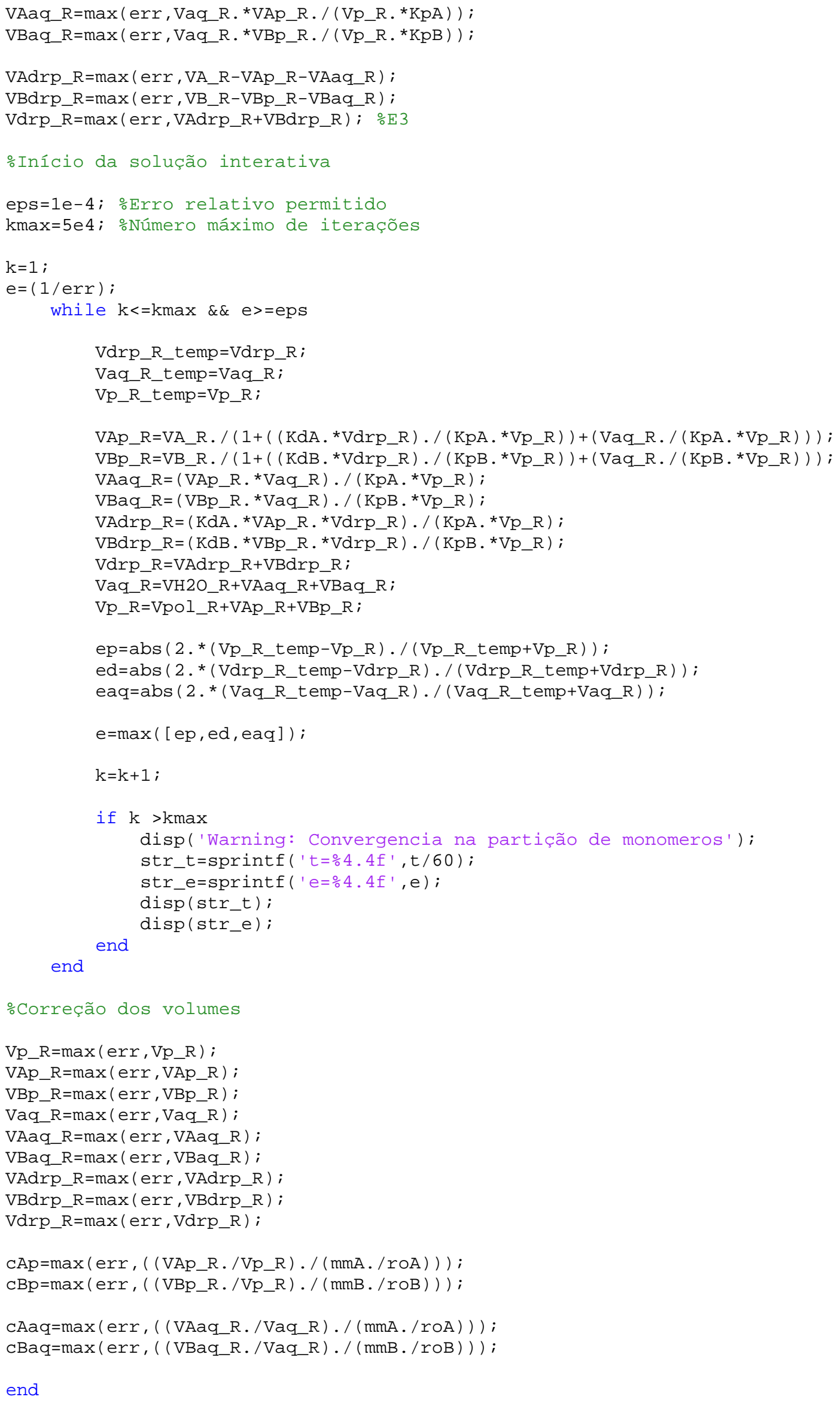




\section{Efeito_gel.m}

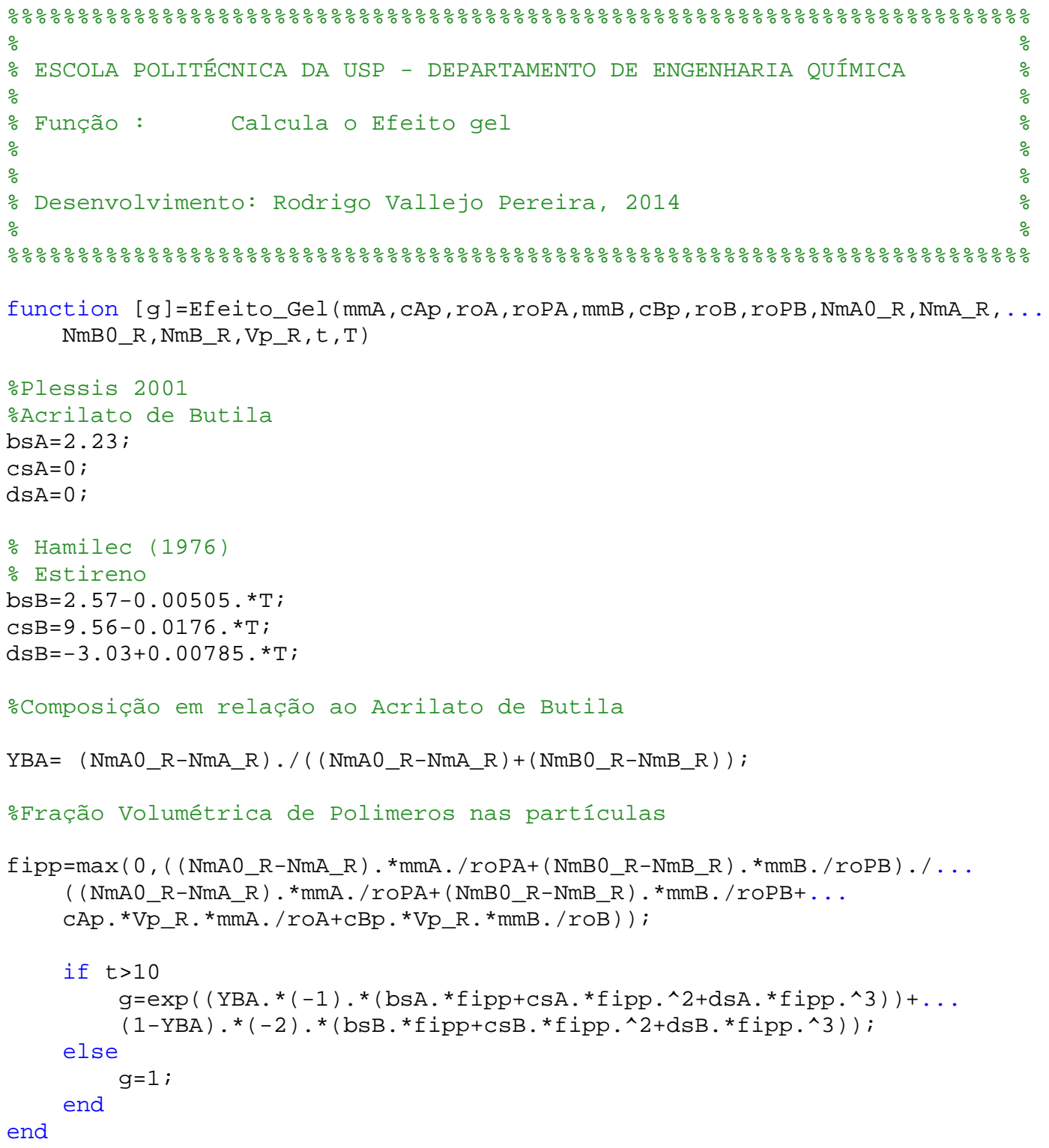




\section{Dec_iniciador.m}

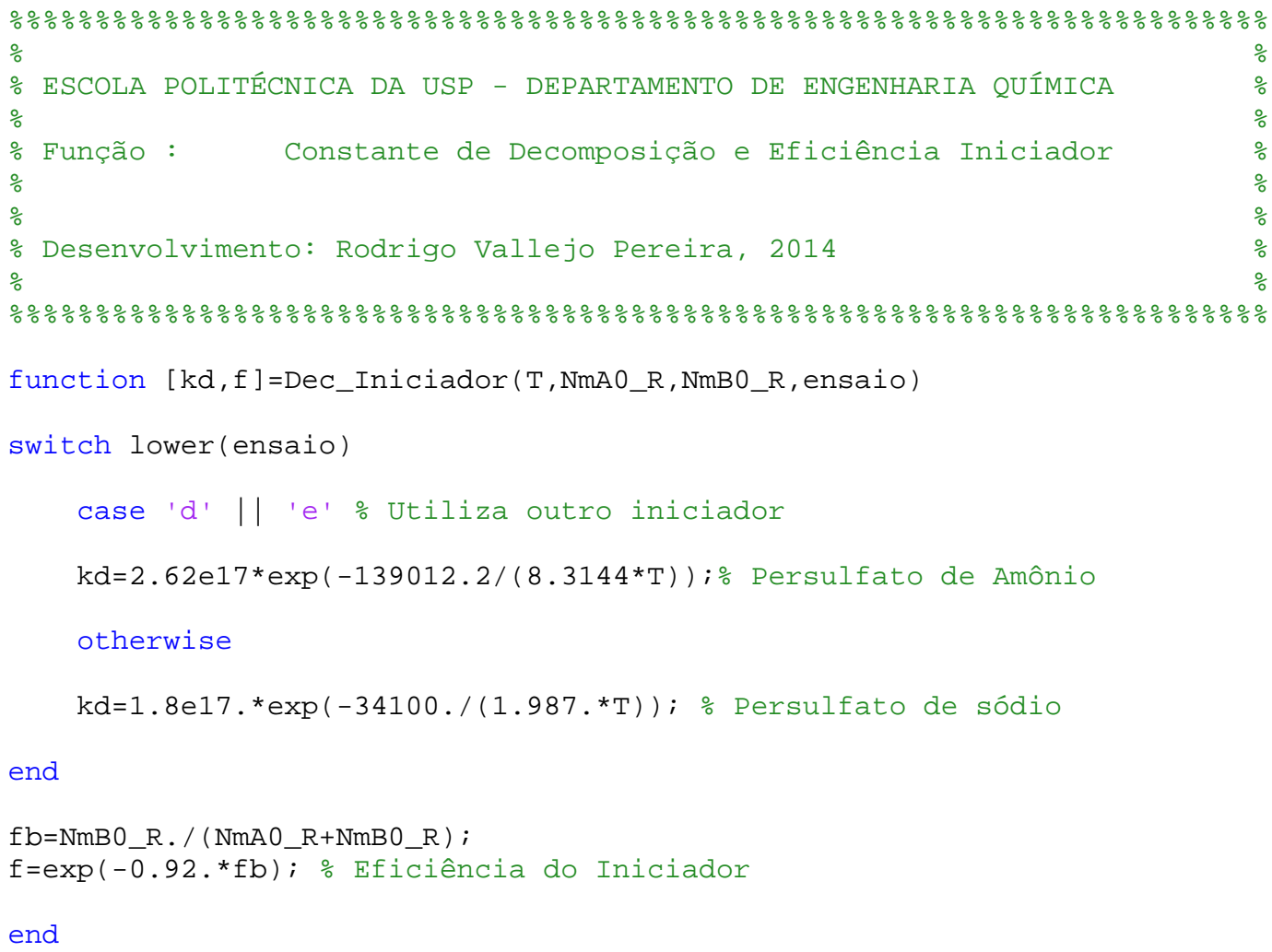




\section{Balanco_radicais.m}

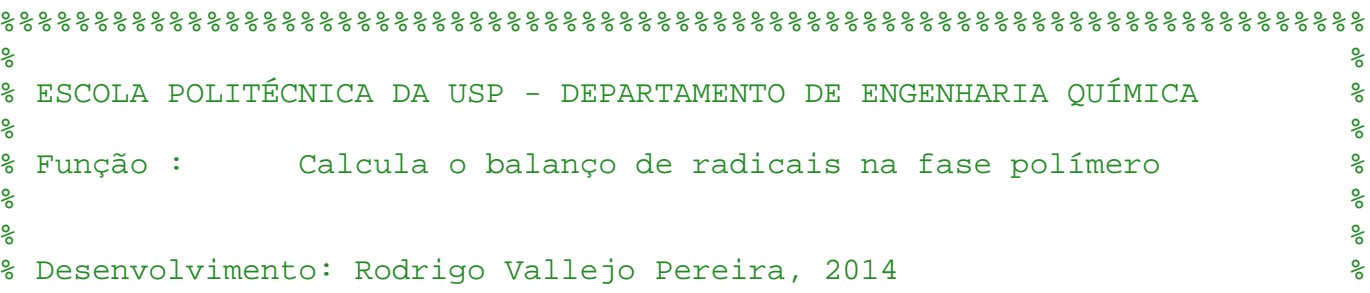




\section{Balanco_Pop.m}

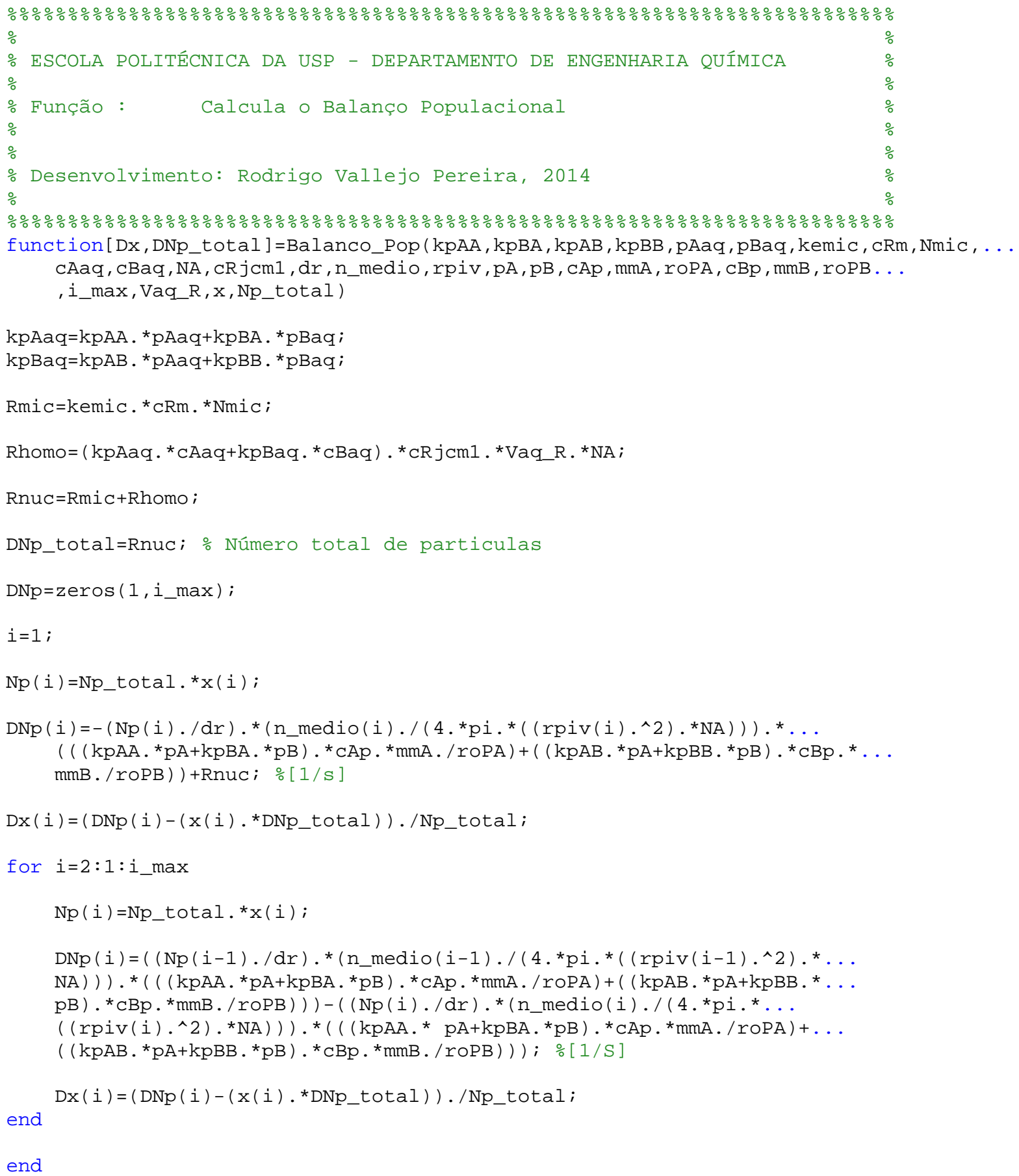

end 


\section{Balanco_momentos.m}

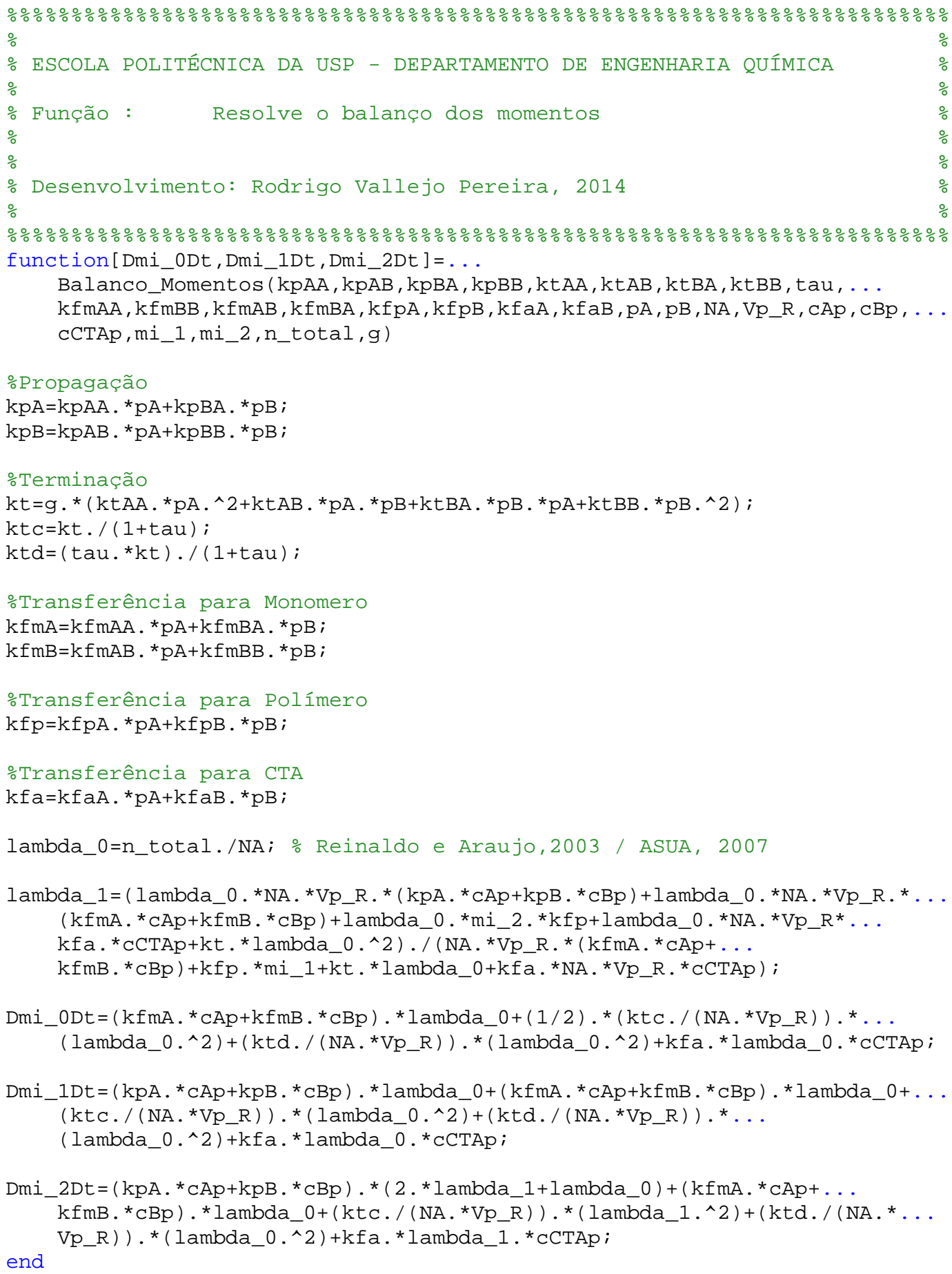




\section{Bal_massa.m}

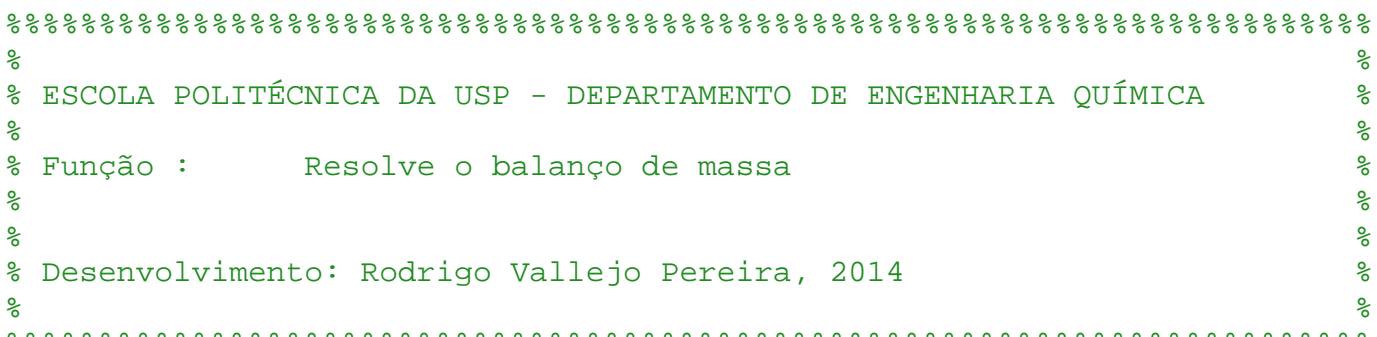

function [DVRDt, DVH2ODt, DNmINIDt, DNADt, DNAADt, DNmBDt, DNmBODt, DNMSDE, ... DNmCTADt, NmCTAp] =Bal_Massa (kpAA, kpAB, kpBA, kpBB, pA, pB, pAaq, pBaq, cAp, ... cBp, cAaq, cBaq, cRTaq, NA, t, t0_F1, t0_F2, tf_F1, tf_F2, F1, $F 2, \operatorname{roPA}, \mathrm{rOA}, \mathrm{mmA}, .$. roPB, roB $, \mathrm{mmB}, \mathrm{fH} 2 \mathrm{O} \_\mathrm{F} 1, \mathrm{fH} 2 \mathrm{O} \_\mathrm{F} 2, \mathrm{kd}, \mathrm{NmINI} R, \mathrm{CINI} F 1, \mathrm{CINI} F 2, \mathrm{CA}$ F1, CA_F2,... CB_F1, CB_F2, CS_F1, CS_F2, n_total, kfaA, kfaB, NmCTA_R, CCTA_F1, CCTA_F2,Vaq_R, ... Vp_R, Vdrp_R, sigma, KpCTA, KCTAdwAd, kfmAA, kfmBB, kfmAB, kfmBA)

$\mathrm{kpA}=\mathrm{kpAA} .{ }^{\star} \mathrm{pA}+\mathrm{kpBA} \cdot{ }^{\star} \mathrm{pB}$;

$\mathrm{kpB}=\mathrm{kpAB} .{ }^{*} \mathrm{pA}+\mathrm{kpBB} .{ }^{*} \mathrm{pB}$;

$\mathrm{kpAaq}=\mathrm{kpAA} .{ }^{*} \mathrm{pAaq}+\mathrm{kpBA} .{ }^{*} \mathrm{pBaq}$;

$\mathrm{kpBaq}=\mathrm{kpAB} .{ }^{*} \mathrm{pAaq}+\mathrm{kpBB} .{ }^{*} \mathrm{pBaq}$;

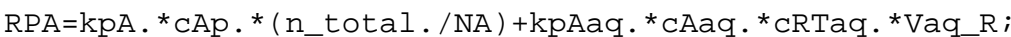

$\mathrm{RPB}=\mathrm{kpB} .{ }^{\star} \mathrm{CBP} . *\left(\mathrm{n} \_\right.$total.$\left./ \mathrm{NA}\right)+\mathrm{kpBaq} \cdot{ }^{\star} \mathrm{CBaq} \cdot{ }^{*} \mathrm{CRTaq} \cdot{ }^{*}$ Vaq_R;

$\mathrm{RFMA}=\left(\mathrm{kfmAA} .{ }^{*} \mathrm{pA}+\mathrm{k} f \mathrm{mBA} \cdot{ }^{*} \mathrm{pB}\right) .{ }^{*} \mathrm{cAp} \cdot{ }^{*}\left(\mathrm{n} \_\right.$total. $\left./ \mathrm{NA}\right)$;

$\mathrm{RFMB}=\left(\mathrm{k} f \mathrm{mAB} \cdot{ }^{\star} \mathrm{pA}+\mathrm{k} f \mathrm{mBB} \cdot{ }^{\star} \mathrm{pB}\right) \cdot{ }^{\star} \mathrm{cBp} \cdot{ }^{*}\left(\mathrm{n} \_t o t a l . / \mathrm{NA}\right)$;

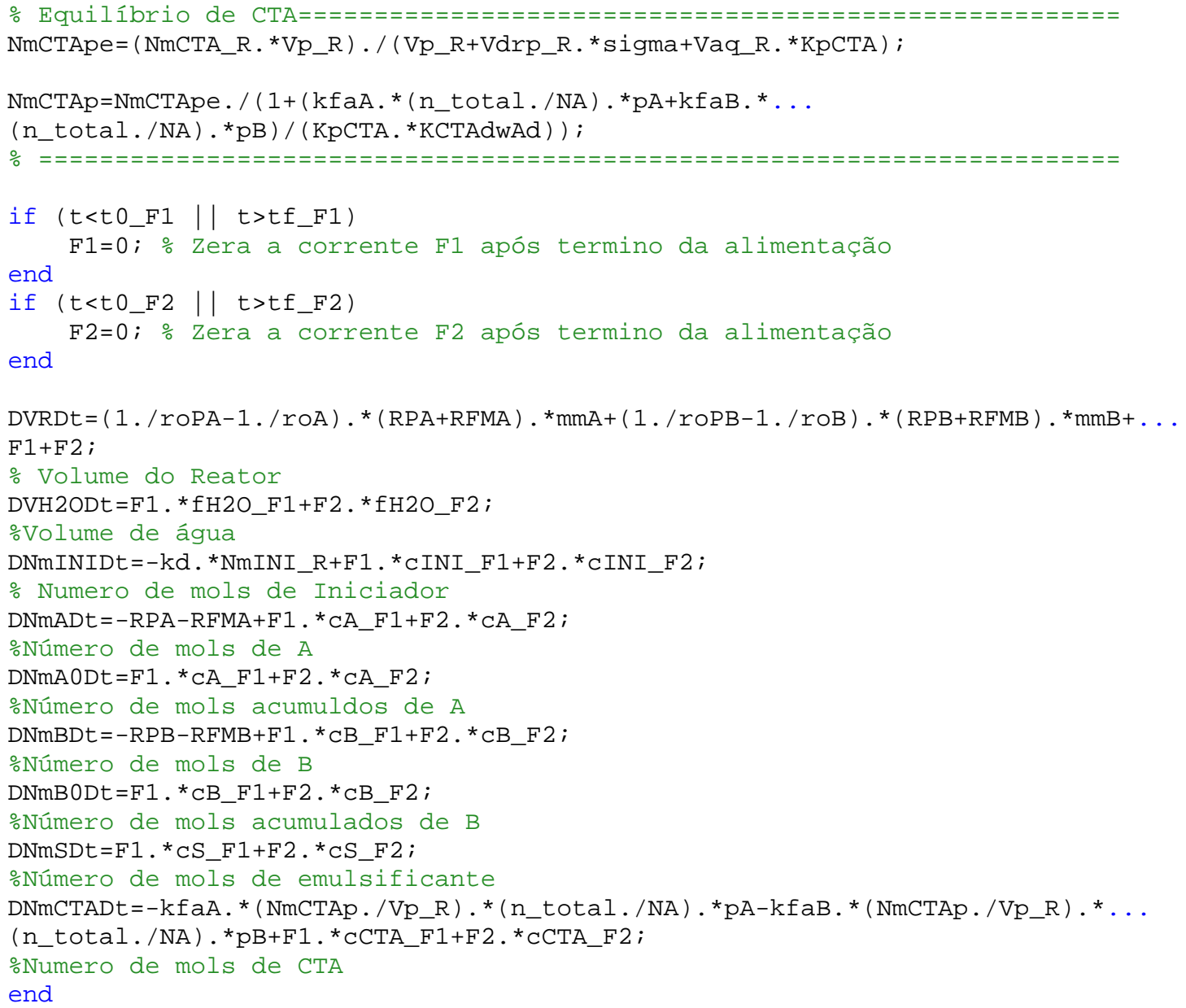

